\title{
MOLECULAR GAS AND STAR FORMATION IN NEARBY DISK GALAXIES
}

\author{
Adam K. Leroy ${ }^{1}$, Fabian Walter ${ }^{2}$, Karin Sandstrom ${ }^{2}$, Andreas Schruba $^{3}$, Juan-Carlos Munoz-Mateos ${ }^{1}$, \\ Frank Bigiel ${ }^{4}$, Alberto Bolatto ${ }^{5}$, Elias Brinks ${ }^{6}$, W. J. G. De Blok ${ }^{7}$, Sharon Meidt ${ }^{2}$, Hans-Walter Rix $^{2}$, \\ Erik Rosolowsky ${ }^{8}$, Eva Schinnerer $^{2}$, KARL-Friedrich Schuster $^{9}$, ANd ANTONio Usero ${ }^{10}$ \\ ${ }^{1}$ National Radio Astronomy Observatory, 520 Edgemont Road, Charlottesville, VA 22903, USA \\ ${ }^{2}$ Max Planck Institute für Astronomie, Königstuhl 17, D-69117 Heidelberg, Germany \\ ${ }^{3}$ California Institute for Technology, 1200 East California Boulevard, Pasadena, CA 91125, USA \\ ${ }^{4}$ Theoretische Astrophysik, Albert-Ueberle-Str. 2, D-69120 Heidelberg, Germany \\ ${ }^{5}$ Department of Astronomy, University of Maryland, College Park, MD USA \\ ${ }^{6}$ Centre for Astrophysics Research, University of Hertfordshire, Hatfield AL10 9AB, UK \\ ${ }^{7}$ Astrophysics, Cosmology and Gravity Centre, Department of Astronomy, University of Cape Town, Private Bag X3, Rondebosch 7701, South Africa \\ ${ }^{8}$ University of British Columbia, Okanagan Campus, Kelowna, BC, Canada \\ ${ }^{9}$ IRAM, 300 rue de la Piscine, F-38406 St. Martin d" Hères, France \\ ${ }^{10}$ Observatorio Astronómico Nacional, C/ Alfonso XII, 3, E-28014 Madrid, Spain \\ Received 2012 April 13; accepted 2012 December 24; published 2013 June 17
}

\begin{abstract}
We compare molecular gas traced by ${ }^{12} \mathrm{CO}(2-1)$ maps from the HERACLES survey, with tracers of the recent star formation rate (SFR) across 30 nearby disk galaxies. We demonstrate a first-order linear correspondence between $\Sigma_{\text {mol }}$ and $\Sigma_{\text {SFR }}$ but also find important second-order systematic variations in the apparent molecular gas depletion time, $\tau_{\mathrm{dep}}^{\mathrm{mol}}=\Sigma_{\mathrm{mol}} / \Sigma_{\mathrm{SFR}}$. At the $1 \mathrm{kpc}$ common resolution of HERACLES, CO emission correlates closely with many tracers of the recent SFR. Weighting each line of sight equally, using a fixed $\alpha_{\mathrm{CO}}$ equivalent to the Milky Way value, our data yield a molecular gas depletion time, $\tau_{\mathrm{dep}}^{\mathrm{mol}}=\Sigma_{\mathrm{mol}} / \Sigma_{\mathrm{SFR}} \approx 2.2 \mathrm{Gyr}$ with $0.3 \mathrm{dex} 1 \sigma$ scatter, in very good agreement with recent literature data. We apply a forward-modeling approach to constrain the power-law index, $N$, that relates the SFR surface density and the molecular gas surface density, $\Sigma_{\mathrm{SFR}} \propto \Sigma_{\text {mol }}^{N}$. We find $N=1 \pm 0.15$ for our full data set with some scatter from galaxy to galaxy. This also agrees with recent work, but we caution that a power-law treatment oversimplifies the topic given that we observe correlations between $\tau_{\text {dep }}^{\text {mol }}$ and other local and global quantities. The strongest of these are a decreased $\tau_{\text {dep }}^{\text {mol }}$ in low-mass, low-metallicity galaxies and a correlation

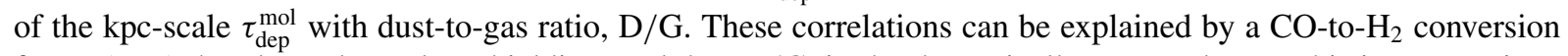
factor $\left(\alpha_{\mathrm{CO}}\right)$ that depends on dust shielding, and thus $\mathrm{D} / \mathrm{G}$, in the theoretically expected way. This is not a unique interpretation, but external evidence of conversion factor variations makes this the most conservative explanation of the strongest observed $\tau_{\text {dep }}^{\text {mol }}$ trends. After applying a D/G-dependent $\alpha_{\mathrm{CO}}$, some weak correlations between $\tau_{\mathrm{dep}}^{\mathrm{mol}}$ and local conditions persist. In particular, we observe lower $\tau_{\text {dep }}^{\text {mol }}$ and enhanced CO excitation associated with nuclear gas concentrations in a subset of our targets. These appear to reflect real enhancements in the rate of star formation per unit gas, and although the distribution of $\tau_{\text {dep }}$ does not appear bimodal in galaxy centers, $\tau_{\text {dep }}$ does appear multivalued at fixed $\Sigma_{\mathrm{H} 2}$, supporting the idea of "disk" and "starburst" modes driven by other environmental parameters.
\end{abstract}

Key words: evolution - galaxies: ISM - galaxies: spiral - ISM: molecules - stars: formation

\section{INTRODUCTION}

The relationship between gas and star formation in galaxies plays a key role in many areas of astrophysics. Its (non)evolution over cosmic time informs our understanding of galaxy evolution at high redshift (Daddi et al. 2010; Tacconi et al. 2010; Genzel et al. 2010). The small-scale efficiency of star formation is a key input to galaxy simulations, and scaling relations measured for whole galaxies provide important benchmarks for the output of these simulations. Measurements of gas and star formation at large scales give context for studies focusing on parts of the Milky Way (e.g., Lada et al. 2010; Heiderman et al. 2010) and the nearest galaxies (e.g., Schruba et al. 2010; Chen et al. 2010). Ultimately, a quantitative understanding of the gas-stars cycle is needed to understand galaxy evolution, with implications for the galaxy luminosity function, the galaxy color-magnitude diagram, the structure of stellar disks, and chemical enrichment among other key topics.

Recent multiwavelength surveys make it possible to estimate the surface densities of gas and recent star formation in dozens of nearby galaxies. This has lead to several studies of the relationship between gas and stars. Many of these focus on a single galaxy (e.g., Heyer et al. 2004; Kennicutt et al. 2007; Blanc et al. 2009; Verley et al. 2010; Rahman et al. 2011) or a small sample (e.g., Wilson et al. 2009; Warren et al. 2010). Restricted by the availability of complete molecular gas maps, studies of large sets of galaxies (e.g., Young et al. 1996; Kennicutt 1998b; Rownd \& Young 1999; Murgia et al. 2002; Leroy et al. 2005; Saintonge et al. 2011) mostly use integrated measurements or a few low-resolution pointings per galaxy.

From 2007-2010, the HERA CO-Line Extragalactic Survey (HERACLES, first maps in Leroy et al. 2009) used the IRAM 30 $\mathrm{m}$ telescope ${ }^{11}$ to construct maps of $\mathrm{CO}$ emission from 48 nearby galaxies. The common spatial resolution of the survey is $\sim 1 \mathrm{kpc}$, sufficient to place many resolution elements across a typical disk galaxy. Because the targets overlap surveys by Spitzer (mostly SINGS and LVL; Kennicutt et al. 2003a; Dale et al. 2009) and

\footnotetext{
11 IRAM is supported by CNRS/INSU (France), the MPG (Germany), and the IGN (Spain).
} 
Galaxy Evolution Explorer (GALEX; mostly the NGS; Gil de Paz et al. 2007), a wide variety of multiwavelength data are available for most targets. In this paper, we take advantage of these data to compare tracers of molecular gas and recent star formation at $1 \mathrm{kpc}$ resolution across a large sample of 30 galaxies.

This paper builds on work by Leroy et al. (2008, hereafter L08) and Bigiel et al. (2008, hereafter B08). They combined the first HERACLES maps with data from The Hi Nearby Galaxies Survey (THINGS; Walter et al. 2008), SINGS, and the GALEX NGS data to compare $\mathrm{H}$ i, $\mathrm{CO}$, and tracers of recent star formation in a sample of nearby galaxies. In the disks of large spiral galaxies, they found little or no dependence of the star formation rate (SFR) per unit molecular gas on environment. The fraction of interstellar gas in the molecular phase, on the other hand, varies strongly within and among galaxies, exhibiting correlations with interstellar pressure, stellar surface density, and total gas surface density among other quantities (Wong \& Blitz 2002; Blitz \& Rosolowsky 2006; L08). They advocated a scenario for star formation in disk galaxies in which star formation in isolated giant molecular clouds (GMCs) is a fairly universal process while the formation of these clouds out of the atomic gas reservoir depends sensitively on environment (see also Wong 2009; Ostriker et al. 2010).

The full HERACLES sample spans a wider range of masses, morphologies, metallicities, and SFRs than the spirals studied in L08 and B08. Schruba et al. (2011) and Bigiel et al. (2011) used these to extend the findings of L08 and B08. Using stacking techniques, Schruba et al. (2011) demonstrated that correlations between star formation tracers and $\mathrm{CO}$ emission extend into the regime where atomic gas dominates the interstellar medium (ISM), $\Sigma_{\mathrm{HI}}>\Sigma_{\text {mol }}$. This provides the strongest evidence yet that star formation in disk galaxies can be separated into star formation from molecular gas and the balance between atomic and molecular gas, a hypothesis that has a long history (e.g., Young \& Scoville 1991 and references therein). Bigiel et al. (2011) demonstrated that a fixed ratio of CO to recent SFR remains a reasonable description of the ensemble of 30 galaxies.

In this paper we expand on L08, B08, Bigiel et al. (2011), and Schruba et al. (2011) and examine the general relationship between molecular gas and SFR in nearby disk galaxies. We divide the analysis into two main parts. In Section 3, we consider the scaling relation linking $\Sigma_{\mathrm{SFR}}$ to $\Sigma_{\mathrm{mol}}$, the molecular analog to the "Kennicutt-Schmidt law" or "star formation law." We show the distribution of data in $\Sigma_{\mathrm{SFR}}-\Sigma_{\mathrm{mol}}$ parameter space using different weightings (Section 3.1) and examine how this distribution changes with different approaches to physical parameter-varying the choice of SFR tracer, the CO transition studied, the processing of SFR maps, or the adopted conversion factor (Section 3.2). Using an expanded version of the Monte Carlo modeling approach of Blanc et al. (2009), we carry out power-law fits to our data, avoiding some of the systematic biases present in previous work (Section 3.3). Finally, we compare our results to a wide collection of literature data, demonstrating an emerging consensus with regard to the region of parameter space occupied by $\Sigma_{\mathrm{SFR}}-\Sigma_{\mathrm{mol}}$ data, if not the interpretation (Section 3.4). We conclude that in the disks of normal, massive star-forming galaxies, to first order the relationship between $\Sigma_{\mathrm{SFR}}$ and $\Sigma_{\text {mol }}$ can be described by a single depletion time with a factor of two scatter. This expands and reinforces the results of L08, B08, and Schruba et al. (2011).

In Section 4, we show important second-order deviations from this simple picture, which can easily be missed by comparing only $\Sigma_{\mathrm{SFR}}$ and $\Sigma_{\mathrm{mol}}$. We find systematic variations in the molecular gas depletion time, $\tau_{\text {dep }}^{\text {mol }}$ as a function of global galaxy properties (Section 4.1) and local conditions (Section 4.2). This analysis includes the first resolved comparison of $\tau_{\mathrm{dep}}^{\mathrm{mol}}$ to the local dust-to-gas ratio, which is expected to play a key role in setting the CO-to- $\mathrm{H}_{2}$ conversion factor, $\alpha_{\mathrm{CO}}$, and we discuss the possibility that $\alpha_{\mathrm{CO}}$ drives some of the observed $\tau_{\text {dep }}^{\mathrm{H} 2}$ variations. We explicitly consider the central regions of our targets (Section 4.4) and show strong evidence for lower $\tau_{\text {dep }}^{\text {mol }}$, i.e., more efficient star formation, in galaxy centers compared to galaxy disks - a phenomenon that we discuss in context of recent proposals for "disk" and "starburst" modes of star formation (Daddi et al. 2010; Genzel et al. 2010). In our bestresolved targets we examine how the scatter in $\tau_{\mathrm{dep}}^{\mathrm{mol}}$ depends on spatial scale (Section 4.3). The relationship appears much shallower than one would expect for uncorrelated averaging in a disk. This suggests either a high degree of large-scale synchronization in the star formation process or, more likely, widespread systematic, but subtle, variations in $\tau_{\mathrm{dep}}^{\text {mol }}$ due to stillundiagnosed drivers.

Thus our conclusions may be abstracted to: molecular gas and star formation exhibit a first order one-to-one scaling but we observe important second order variations about this scaling. These include likely conversion factor effects, efficient nuclear starbursts, and weak systematic variations in $\tau_{\text {dep }}^{\text {mol that }}$ emerge considering scale-dependent scatter or global galaxy properties. The remainder of this section presents a brief background, Section 2 describes our data and physical parameter estimation, Sections 3 and 4 motivate these conclusions, and Section 5 synthesizes these results and identifies several key future directions.

\subsection{Background}

Following Schmidt (1959, 1963), astronomers have studied the relationship between gas and the SFR for more than 50 years. Most recent work follows Kennicutt (1989, 1998b) and compares the surface densities of SFR, $\Sigma_{\mathrm{SFR}}$, and neutral $\left(\mathrm{HI}+\mathrm{H}_{2}\right)$ gas mass, $\Sigma_{\text {gas }}$. Recent work focuses heavily on the power-law relationship between these surface densities (the "Schmidt-Kennicutt law" or the "star formation law"):

$$
\Sigma_{\mathrm{SFR}}=A \times \Sigma_{\mathrm{gas}}{ }^{N} .
$$

An alternative approach treats the ratio of gas and SFR as the quantity of interest (Young et al. 1986, 1996; L08). This ratio can be phrased as a gas depletion time $\left(\Sigma_{\text {mol }} / \Sigma_{\text {SFR }}\right)$ or its inverse, a star formation efficiency $\left(\Sigma_{\mathrm{SFR}} / \Sigma_{\mathrm{mol}}\right)$. These share the same physical meaning, which is the SFR per unit gas. Both convolve a timescale with a true efficiency, for example the lifetime of a molecular cloud with the fraction of gas converted to stars over this time. We focus exclusively on molecular gas in this paper and phrase this ratio as the molecular gas depletion time,

$$
\tau_{\mathrm{dep}}^{\mathrm{mol}}=\frac{\Sigma_{\mathrm{mol}}}{\Sigma_{\mathrm{SFR}}},
$$

which is the time for star formation to consume the current molecular gas supply.

The state of the field is roughly the following. Kennicutt (1998b) demonstrated a tight, nonlinear $(N=1.4 \pm 0.15)$ scaling between galaxy-averaged $\Sigma_{\mathrm{SFR}}$ and $\Sigma_{\text {gas }}$ spanning from normal disk galaxies to starbursts. Including $\mathrm{H}_{\mathrm{I}}$ improved the agreement between disks and starbursts, but most of the dynamic 
range and the nonlinear slope were driven by the contrast between the disks and merger-induced starbursts, especially the local ultraluminous infrared galaxy (ULIRG) population. The adoption of a single conversion factor for all systems also had significant impact; adopting the "ULIRG" conversion factor suggested by Downes \& Solomon (1998) for the Kennicutt (1998b) starburst data drives the implied slope to $N \sim 1.7$. Subsequent studies resolved galaxy disks-often as radial profiles - and usually revealed distinct relationships between $\Sigma_{\mathrm{SFR}}, \Sigma_{\mathrm{HI}}$, and $\Sigma_{\text {mol }}$ with shallower indices $N$ for $\mathrm{H}_{2}$ than $\mathrm{HI}$ (Wong \& Blitz 2002; Heyer et al. 2004; Kennicutt et al. 2007; Schruba et al. 2011; B08; L08). This suggests that the immediate link is between SFR and $\mathrm{H}_{2}$. A more aggressive conclusion, motivated by the steep, relatively weak relation between $\Sigma_{\text {SFR }}$ and $\Sigma_{\mathrm{H}_{\mathrm{I}}}$, is that star formation in galaxy disks may be broken into two parts: (1) the formation of stars in molecular clouds and (2) the balance between $\mathrm{H}_{2}$ and $\mathrm{H}$ I (Wong \& Blitz 2002; Blitz \& Rosolowsky 2006; L08; B08).

The fraction of dense molecular gas also appears to be a key parameter. Gao \& Solomon (2004) found a roughly fixed ratio of SFR to HCN emission, a dense gas tracer, extending from spiral galaxies to starbursts. Over the same range, the ratio of SFR to total $\mathrm{H}_{2}$, traced by $\mathrm{CO}$ emission, varies significantly (though the two relate roughly linearly in their normal galaxy sample). Galactic studies also highlight the impact of density on the SFR on cloud scales (Wu et al. 2005; Heiderman et al. 2010; Lada et al. 2010). It remains unclear how the dense gas fraction varies inside galaxy disks, but merger-induced starbursts do show high HCN-to-CO ratios (Gao \& Solomon 2004; García-Burillo et al. 2012).

SFR tracers and CO can both be observed at high redshift. Genzel et al. (2010) demonstrated broad consistency between local $\mathrm{H}_{2}-\mathrm{SFR}$ relations and those at $z \sim 1-3$. One key difference

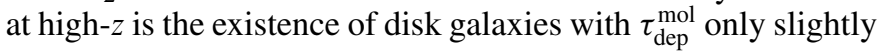
lower than those in local disk galaxies but $\mathrm{H}_{2}$ surface density, $\Sigma_{\text {mol }}$, as high as that found in starbursts in the local universe (Daddi et al. 2010; Tacconi et al. 2010). Merger-driven starbursts with similar $\Sigma_{\text {mol }}$ can have much lower $\tau_{\text {dep }}^{\text {mol }}$, suggesting the relevance of another parameter to set $\tau_{\text {dep }}^{\text {mol }}$. Density and the dynamical timescale are both good candidates (Daddi et al. 2010; Genzel et al. 2010).

Meanwhile, investigations of the Milky Way and the nearest galaxies have attempted to connect observed scaling relations to the properties of individual star-forming regions. These are able to recover the galaxy-scale relations at large scale but find enormous scatter in the ratios of SFR tracers to molecular gas on small scales (Schruba et al. 2010; Chen et al. 2010; Onodera et al. 2010). Detailed studies of Milky Way and LMC clouds suggest the time-evolution of individual star-forming regions as a likely source of this scatter (Murray 2010; Kawamura et al. 2009), with the volume density of individual clouds a key parameter (Heiderman et al. 2010; Lada et al. 2010).

For additional background we refer the reader to the recent review by Kennicutt \& Evans (2012).

\section{DATA}

\subsection{Data Sets}

We use HERACLES CO (2-1) maps to infer the distribution of $\mathrm{H}_{2}$ and GALEX far-ultraviolet (FUV), Spitzer infrared (IR), and literature $\mathrm{H} \alpha$ data to trace recent star formation. We supplement these with $\mathrm{HI}$ data used to mask the $\mathrm{CO}$, derive kinematics, and measure the dust-to-gas ratio with near-IR data used to estimate the stellar surface density, $\Sigma_{*}$.

HERACLES CO. The HERA CO Line Extragalactic Survey (HERACLES) used the Heterodyne Receiver Array (HERA; Schuster et al. 2004) on the IRAM $30 \mathrm{~m}$ telescope to map CO (2-1) emission from 48 nearby galaxies, of which we use 30 in this paper (see Section 2.3). HERACLES combines an IRAM Large Program and several single-semester projects that spanned from 2007 to 2010. Leroy et al. (2009) presented the first maps (see also Schuster et al. 2007). The additional data here were observed and reduced in a similar manner. The largest change is a revised estimate of the main beam efficiency, lowering observed intensities by $\approx 10 \%$. This propagates to a revised CO (2-1)/(1-0) line ratio estimate, so our estimates of $\Sigma_{\text {mol }}$ are largely unaffected compared to B08 and L08. The HERACLES cubes cover out to radii of $r_{25}$ with angular resolution $13^{\prime \prime}$ and typical $1 \sigma$ sensitivity $20 \mathrm{mK}$ per $5 \mathrm{~km} \mathrm{~s}^{-1}$ channel.

We integrate each cube along the velocity axis to produce maps of the integrated intensity along each line of sight. We wish to avoid including unnecessary noise in this integral and so restrict the velocity range over which we integrate to be as small as possible while still containing the $\mathrm{CO}$ line, i.e., we "mask" the cubes. To be included in the mask a pixel must meet one of two conditions: (1) lie within $\pm 25 \mathrm{~km} \mathrm{~s}^{-1}$ of the local mean H I velocity (derived from THINGS; Walter et al. 2008, supplemented by new and archival $\mathrm{H}_{\mathrm{I}}$ ) or (2) lie in part of the spectrum near either two consecutive channels with signal-tonoise ratio $(\mathrm{S} / \mathrm{N})$ above 4 or three consecutive channels with $\mathrm{S} / \mathrm{N}$ above 3 . Condition (2) corresponds to traditional radio masking (e.g., Helfer et al. 2003; Walter et al. 2008). Condition (1) is less conventional, but important to our analysis. Integrating over the Hi line, which is detected throughout our targets, guarantees that we have an integrated intensity measurement along each line of sight, even lines of sight that lack bright $\mathrm{CO}$ emission (see Schruba et al. 2011, for detailed discussion of this approach). This avoids a traditional weakness of masking, that nondetections are difficult to deal with quantitatively. We calculate maps of the statistical uncertainty in the integrated $\mathrm{CO}$ intensity from the combination of the mask and estimates of the noise derived from signal-free regions. The result is an integrated intensity and associated uncertainty for each line of sight in the HERACLES mask.

SINGS and LVL IR. We use maps of IR emission from 3.6-160 $\mu \mathrm{m}$ from the Spitzer Infrared Nearby Galaxies Survey (SINGS; Kennicutt et al. 2003a) and the Local Volume Legacy survey (LVL; Dale et al. 2009). We describe the processing of these maps in Leroy et al. (2012, hereafter L12).

SINGS, LVL, and Literature $H \alpha$. Both SINGS and LVL published continuum-subtracted $\mathrm{H} \alpha$ images for most of our sample. We supplement these with literature maps, particularly from the GoldMine and Palomar-Las Campanas surveys. L12 describe our approach to these maps (masking, N II correction, flux scaling, background subtraction) and list the source of the $\mathrm{H} \alpha$ data for each galaxy.

GALEX UV. For 24 galaxies, we use NUV and FUV maps from the Nearby Galaxy Survey (NGS; Gil de Paz et al. 2007). For one galaxy, we use a map from the Medium Imaging Survey (MIS) and we take maps for five targets from the All-sky Imaging Survey (AIS). L12 describe our processing.

BIMA $+12 m$ and NRO $45 \mathrm{~m} C O$ (1-0) Maps. A subset of our targets have also been observed by the BIMA SONG (Helfer et al. 2003) or the Nobeyama CO Atlas of Nearby Spiral 
Galaxies (Kuno et al. 2007). Where these data are available, we apply our HERACLES masks to these maps and measure CO (1-0) intensity. We only use BIMA SONG maps that include short-spacing data from the Kitt Peak $12 \mathrm{~m}$.

THINGS and Supplemental $H$ I . We assemble H I maps for all targets, which we use to mask the $\mathrm{CO}$, estimate the dust-to-gas ratio, explore $24 \mu \mathrm{m}$ cirrus corrections, and derive approximate rotation curves. These come from THINGS (Walter et al. 2008) and a collection of new and archival Very Large Array data (programs AL731 and AL735). These supplemental H I are C+D configuration maps with resolutions $13^{\prime \prime}-25^{\prime \prime}$. We reduced and imaged these in a standard way using the CASA package.

\subsection{Physical Parameter Estimates}

Following standard practice in this field, we estimate physical parameters from observables. Despite the intrinsic uncertainty involved in this process, these estimates play a fundamental role in enhancing our understanding of the physics of galaxy and star formation, as demonstrated from the earliest works in this subfield (Young \& Knezek 1989; Kennicutt 1989). We adopt an approach largely oriented to physical quantities, but discuss the impact of our assumptions throughout.

$\mathrm{CO}$ Intensity to $\mathrm{H}_{2}$. We convert $\mathrm{CO}(2-1)$ intensity to $\mathrm{H}_{2}$ mass via

$$
\Sigma_{\mathrm{mol}}\left[M_{\odot} \mathrm{pc}^{-2}\right]=6.3\left(\frac{0.7}{R_{21}}\right)\left(\frac{\alpha_{\mathrm{CO}}^{1-0}}{4.35}\right) I_{\mathrm{CO}}\left[\mathrm{K} \mathrm{km} \mathrm{s}^{-1}\right],
$$

where $R_{21}$ is the $\mathrm{CO}(2-1)$-to- $\mathrm{CO}(1-0)$ line ratio and $\alpha_{\mathrm{CO}}$ is the $\mathrm{CO}(1-0)-$ to- $\mathrm{H}_{2}$ conversion factor. By default, we adopt a Galactic conversion factor, $\alpha_{\mathrm{CO}}^{1-0}=4.35 M_{\odot} \mathrm{pc}^{-2}\left(\mathrm{~K} \mathrm{~km} \mathrm{~s}^{-1}\right)^{-1}$ equivalent to $X_{\mathrm{CO}}=2 \times 10^{20} \mathrm{~cm}^{-2}\left(\mathrm{~K} \mathrm{~km} \mathrm{~s}^{-1}\right)^{-1}$ (Strong \& Mattox 1996; Dame et al. 2001) and a line ratio of $R_{21}=0.7$. This line ratio is slightly lower than the $R_{21}=0.8$ derived by Leroy et al. (2009), reflecting the revised efficiency used in the reduction. Appendix A motivates this value using integrated flux ratios and follow-up spectroscopy of HERACLES targets. Equation (3) and all " $\Sigma_{\text {mol }}$ " in this paper include a factor of 1.36 to account for helium. Because we consider only molecular gas, any results that we derive using a fixed $\alpha_{\mathrm{CO}}$ can be straightforwardly restated in terms of CO intensity.

We adopt this "Galactic" $\alpha_{\mathrm{CO}}^{1-0}$ to facilitate clean comparison to previous work, but improved estimates exist for HERACLES. Sandstrom et al. (2012) solved directly for the CO-to- $\mathrm{H}_{2}$ conversion factor across the HERACLES sample using dust as an independent tracer of the gas mass. They find a somewhat lower average $\alpha_{\mathrm{CO}}^{1-0} \approx 3.1$. We quote this as a CO (1-0) conversion factor, though Sandstrom et al. (2012) directly solve for the $\mathrm{CO}(2-1)$ conversion factor. They find a CO (2-1) conversion factor of $\alpha_{\mathrm{CO}}^{2-1} \approx 4.4 M_{\odot} \mathrm{pc}^{-2}\left(\mathrm{~K} \mathrm{~km} \mathrm{~s}^{-1}\right)^{-1}$, compared to our "Galactic" CO (2-1) conversion factor $\alpha_{\mathrm{CO}}^{2-1}=6.3 M_{\odot} \mathrm{pc}^{-2}$ $\left(\mathrm{K} \mathrm{km} \mathrm{s}^{-1}\right)^{-1}$. Sandstrom et al. (2012) find $\approx 0.4$ dex point-topoint scatter, of which $\approx 0.3$ may be intrinsic with the remainder solution uncertainties. Because Sandstrom et al. (2012) solve directly for a CO (2-1) conversion factor using the same HERACLES data employed in this paper, these values should be borne in mind when reading our results. Our results remain pinned to a Galactic $\mathrm{CO}(1-0)$ conversion factor of $\alpha_{\mathrm{CO}}^{1-0}=4.35 M_{\odot} \mathrm{pc}^{-2}$ $\left(\mathrm{K} \mathrm{km} \mathrm{s}^{-1}\right)^{-1}$ that may be $\approx 30 \%$ too high, on average. As a result, a systematic bias of $\approx 30 \%$ in $\Sigma_{\text {mol }}$ appears plausible with factor of two variations in the conversion factor point-to-point.
In addition to a fixed conversion $\alpha_{\mathrm{CO}}$, we consider the effects of variations in $\alpha_{\mathrm{CO}}$ due to decreased dust shielding at low metallicity and variations in the line width, optical depth, and temperature of $\mathrm{CO}$ in galaxy centers. Our "variable" $\alpha_{\mathrm{CO}}$ builds on the work of Sandstrom et al. (2012), who compare H I, CO (2-1), and $\Sigma_{\text {Dust }}$ in 22 HERACLES galaxies and Wolfire et al. (2010), who consider the effects of dust shielding on the "CO-dark" layer of molecular clouds, where most $\mathrm{H}$ is $\mathrm{H}_{2}$. The $\alpha_{\mathrm{CO}}$ prescription combines three terms

$$
\alpha_{\mathrm{CO}}=\alpha_{\mathrm{CO}}^{0} c_{\mathrm{CO}-\text { dark }}(\mathrm{D} / \mathrm{G}) c_{\text {center }}\left(r_{\mathrm{gal}}\right) .
$$

Here $\alpha_{\mathrm{CO}}^{0}=6.3 M_{\odot} \mathrm{pc}^{-2}\left(\mathrm{~K} \mathrm{~km} \mathrm{~s}^{-1}\right)^{-1}$ is our fiducial CO (2-1) conversion factor in the disk of a galaxy at solar metallicity (Equation (3)). The term $c_{\mathrm{CO}-\text { dark }}$ represents a correction to the $\mathrm{H}_{2}$ mass to reflect the $\mathrm{H}_{2}$ in a CO-dark layer not directly traced by $\mathrm{CO}$ emission. We calculate this factor following Wolfire et al. (2010), assuming that all GMCs share a fixed surface density, $\left\langle\Sigma_{\mathrm{GMC}}\right\rangle$, and adopting a linear scaling between the dust-to-gas ratio and metallicity (see also Glover \& Mac Low 2011). In this case

$$
c_{\mathrm{CO}-\mathrm{dark}}\left(\mathrm{D} / \mathrm{G}^{\prime}\right) \approx 0.65 \exp \left(\frac{0.4}{\mathrm{D} / \mathrm{G}^{\prime} \Sigma_{100}}\right) .
$$

Here $\mathrm{D} / \mathrm{G}^{\prime}$ is normalized to our adopted "Galactic" value of 0.01 , with the normalization constructed to yield $\alpha_{\mathrm{CO}}=\alpha_{\mathrm{CO}}^{0}$ for $\mathrm{D} / \mathrm{G}^{\prime}=1 . \Sigma_{100}=\left\langle\Sigma_{\mathrm{GMC}}\right\rangle / 100 M_{\odot} \mathrm{pc}^{-2}$. Appendix B presents this calculation in detail.

We consider two cases: $\left\langle\Sigma_{\mathrm{GMC}}\right\rangle=100 M_{\odot} \mathrm{pc}^{-2}(" \Sigma=$ $100 ")$ and $\left\langle\Sigma_{\mathrm{GMC}}\right\rangle=50 M_{\odot} \mathrm{pc}^{-2}$ (" $\left.\Sigma=50 "\right)$. " $\Sigma=100 "$ reflects a typical surface density that is often assumed and observed for extragalactic GMCs (e.g., Bolatto et al. 2008; Narayanan et al. 2012). Over the range of $D / G^{\prime}$ that we consider, this prescription reasonably resembles the shallow power-law dependences of $\alpha_{\mathrm{CO}}$ on metallicity calculated from simulations by Feldmann et al. (2012) and Narayanan et al. (2012), who both suggest $\alpha_{\mathrm{CO}} \sim Z^{-0.7}$. " $\left\langle\Sigma_{\mathrm{GMC}}\right\rangle=50 M_{\odot} \mathrm{pc}^{-2}$ " yields a steeper dependence of $\alpha_{\mathrm{CO}}$ on metallicity over our range of interest. Low surface density GMCs or significant contribution of "translucent" ( $\left.A_{V} \sim 1-2 \mathrm{mag}\right)$ gas to the overall CO emission are supported by observations of the Milky Way by Heyer et al. (2009) and Liszt et al. (2010), LMC observations by Hughes et al. (2010), Wong et al. (2011), and M31 (A. Schruba et al., in preparation), but may not be appropriate for more actively star-forming systems (Hughes et al. 2013). We return to this issue in Section 4.

We calculate $\alpha_{\mathrm{CO}}\left(\mathrm{D} / \mathrm{G}^{\prime}\right)$ using $\mathrm{D} / \mathrm{G}^{\prime}$ derived for fixed $\alpha_{\mathrm{CO}}$. Given observations of $\Sigma_{\mathrm{HI}}, I_{\mathrm{CO}}, \Sigma_{\text {dust }}$, and a prescription for $\alpha_{\mathrm{CO}}\left(\mathrm{D} / \mathrm{G}^{\prime}\right)$, one can simultaneously solve for $\alpha_{\mathrm{CO}}$ and $\mathrm{D} / \mathrm{G}^{\prime}$. The solution is often multivalued and unstable, though not intractable. However, after experimentation and comparison with the self-consistent Herschel-based results of Sandstrom et al. (2012), we found that the process does not clearly improve our estimates. In the interests of clarity and simplicity, we work with only $\mathrm{D} / \mathrm{G}$ calculated using fixed $\alpha_{\mathrm{CO}}$ throughout the paper. This simplification biases our $\alpha_{\mathrm{CO}}$ estimate high by $\approx 8 \%$ (" $\Sigma=100 "$ ) and $\approx 15 \%$ (" $\Sigma=50 ")$. With improved $\Sigma_{\text {dust }}$ estimates, we expect that the self-consistent treatment will become necessary.

The third term, $c_{\text {center }}$, accounts for depressed values of $\alpha_{\mathrm{CO}}$ in the centers of galaxies. Sandstrom et al. (2012) find such depressions in the centers of many systems (see also Israel 2009a, 2009b). These likely reflect the same line-broadening and 
temperature effects that drive the commonly invoked "ULIRG conversion factor" (Downes \& Solomon 1998), though the depression observed by Sandstrom et al. (2012) has lower magnitude than the factor of five depression found by Downes \& Solomon (1998). Sandstrom et al. (2012) could not identify a unique observational driver for these depressions, though they correlate well with stellar surface density. Instead, they appear to be present with varying magnitudes in the centers of most systems with bright central $\mathrm{CO}$ emission. Following their recommendation, we apply this correction where $r_{\text {gal }}<0.1 r_{25}$ in systems that have such central CO concentrations. Whenever available, we adopt $c_{\text {center }}$ directly from Sandstrom et al. (2012), taking the factor by which the central $\alpha_{\mathrm{CO}}$ falls below the mean for the disk of that galaxy. For systems with central $\mathrm{CO}$ concentrations but not in the sample of Sandstrom et al. (2012), we apply a factor of two depression, again following their recommendations. Appendix B presents additional details.

SFR from $H \alpha, U V$, and $24 \mu \mathrm{m}$ Emission. L12 combined $\mathrm{UV}, \mathrm{H} \alpha$, and IR emission to estimate the recent SFR surface density, $\Sigma_{\mathrm{SFR}}$, at $1 \mathrm{kpc}$ resolution (the limiting common physical resolution of the HERACLES survey) for our sample. We adopt their estimates and refer the reader to that work for detailed discussion. Briefly, our baseline estimate of $\Sigma_{\text {SFR }}$ combines $\mathrm{H} \alpha$ and infrared emission at $24 \mu \mathrm{m}$ via

$$
\begin{aligned}
& \Sigma_{\mathrm{SFR}}\left[M_{\odot} \mathrm{yr}^{-1} \mathrm{kpc}^{-2}\right]=634 I_{\mathrm{H} \alpha}\left[\mathrm{erg} \mathrm{s} \mathrm{sr}^{-1}\right] \\
& \quad+0.00325 I_{24 \mu \mathrm{m}}\left[\mathrm{MJy} \mathrm{sr}^{-1}\right],
\end{aligned}
$$

where $I_{\mathrm{H} \alpha}$ and $I_{24 \mu \mathrm{m}}$ refer to the line-integrated $\mathrm{H} \alpha$ intensity and intensity at $24 \mu \mathrm{m}$.

The $\mathrm{H} \alpha$ emission captures direct emission from $\mathrm{H}$ II regions powered by massive young stars while the $24 \mu \mathrm{m}$ emission accounts for recent star formation obscured by dust. Before estimating $\Sigma_{\mathrm{SFR}}$, we correct our $24 \mu \mathrm{m}$ maps for the effects of heating of dust by a weak, pervasive radiation field (i.e., a "cirrus") with magnitude derived from modeling the infrared spectral energy distribution (SED). The cirrus removed corresponds to the expected emission from the local dust mass illuminated by a quiescent radiation field, typically $\sim 0.6$ times the Solar neighborhood interstellar radiation field (see L12 for details). We derive the appropriate weighting for the combination of $\mathrm{H} \alpha$ and $24 \mu \mathrm{m}$ emission based on comparing our processed $\mathrm{H} \alpha$ and $24 \mu \mathrm{m}$ maps to literature estimates of $\mathrm{H} \alpha$ extinction. The resulting linear combination resembles that of Kennicutt et al. (2007) but places slightly more weight on the $24 \mu \mathrm{m}$ term. For comparison, we also estimate $\Sigma_{\mathrm{SFR}}$ from combining FUV and $24 \mu \mathrm{m}$ emission and taking $\mathrm{H} \alpha$ alone while assuming a typical 1 magnitude of extinction.

L12 estimate a substantial uncertainty in the absolute calibration of "hybrid" UV+IR or H $\alpha+$ IR tracers, with magnitude $\approx 50 \%$. In addition to this overall uncertainty in the calibration, they derive a point-to-point uncertainty in $\Sigma_{\mathrm{SFR}}$ of $\approx 0.15 \mathrm{dex}$ based on intercomparison of different estimates.

Dust Properties. In order to measure dust properties, we convolve the Spitzer 24, 70, and $160 \mu \mathrm{m}$ data and the CO and H I maps to the resolution of the Spitzer $160 \mu \mathrm{m}$ data. At this resolution, we build radial profiles of each band and then fit the dust models of Draine \& Li (2007) to these profiles. These fits, presented in L12, provide us with radial estimates of the dust-to-gas ratio, $\mathrm{D} / \mathrm{G}$, and are used to help account for "cirrus" contamination when estimating $\Sigma_{\mathrm{SFR}}$. Note that the $\sim 40^{\prime \prime}$ resolution of the $160 \mu \mathrm{m}$ data used to measure these dust properties is significantly coarser than the $1 \mathrm{kpc}$ resolution used for the rest of our data. Where possible, we have compared our Spitzer-based dust masses to masses estimated using the improved SED coverage offered by Herschel (e.g., Aniano et al. 2012); above $\Sigma_{\text {dust }} \approx 0.05 M_{\odot} \mathrm{pc}^{-2}$ the median offset between the Herschel- and Spitzer-based dust masses is only $\approx 10 \%$; however, the dust masses derived for individual rings using only Spitzer do scatter by $\approx 0.3$ dex (a factor of two) compared to Herschel-based dust masses and show weak systematic trends with the sense that Spitzer underestimates the mass of cooler $(\sim 15 \mathrm{~K})$ dust in the outskirts of galaxies (both consistent with the analysis of Draine et al. 2007). We expect that once Herschel images become available, they will significantly improve the accuracy of the dust-based portion of this analysis.

Stellar Mass. To estimate the stellar mass for whole galaxies, we draw $3.6 \mu \mathrm{m}$ fluxes from Dale et al. (2007, 2009), convert to a luminosity using our adopted distance, and apply a fixed $3.6 \mu \mathrm{m}$ mass-to-light ratio. Based on comparison to Zibetti et al. (2009), we use

$$
\Sigma_{*}\left[M_{\odot} \mathrm{pc}^{-2}\right]=200 I_{3.6}\left[\mathrm{MJy} \mathrm{sr}^{-1}\right],
$$

which is $\sim 30 \%$ lower than L08. This value is uncertain by $\sim 50 \%$.

We estimate the stellar surface density, $\Sigma_{*}$, for each kpc-sized element from the contaminant-corrected $3.6 \mu \mathrm{m}$ maps of Meidt et al. (2012). Starting from a reprocessing of the SINGS data (as part of the S4G survey; Sheth et al. 2010), they used independent component analysis to remove contamination by young stars and hot dust from the overall maps. These contaminants make a minor contribution to the overall $3.6 \mu \mathrm{m}$ flux but may be important locally. We convert the contaminant-corrected $3.6 \mu \mathrm{m}$ maps to $\Sigma_{*}$ estimates using Equation (7).

Rotation Velocities. Following L08 and Boissier et al. (2003) we work with a simple two-parameter fit to the rotation curve of each galaxy

$$
v_{\text {rot }}\left(r_{\text {gal }}\right)=v_{\text {flat }}\left[1-\exp \left(\frac{-r_{\text {gal }}}{l_{\text {flat }}}\right)\right],
$$

with $v_{\text {flat }}$ and $l_{\text {flat }}$ free parameters. We derive these from fits to the rotation curves of de Blok et al. (2008) wherever they are available. Where these are not available, we carry out our own tilted ring fits to the combined $\mathrm{H}_{\mathrm{I}}$ and $\mathrm{CO}$ first moment maps. We use these fits to calculate the orbital time $\tau_{\text {orb }}=2 \pi r_{\text {gal }} / v_{\text {rot }}$ for each line of sight.

\subsection{Sample and Galaxy Properties}

We present measurements for galaxies meeting the following criteria: (1) a HERACLES CO map containing a clear CO detection $(\mathrm{S} / \mathrm{N}>5$ over a significant area and multiple channels), (2) Spitzer data at $24 \mu \mathrm{m}$, and (3) inclination $\lesssim 75^{\circ}$. The first condition excludes low-mass galaxies without $\mathrm{CO}$ detections (these are discussed in Schruba et al. 2012). The second removes a few targets with saturated or incomplete Spitzer coverage. The third removes a handful of edge-on galaxies. We are left with the 30 disk galaxies listed in Table 1.

For each target, Table 1 gives the distance, physical resolution of the HERACLES maps at that distance, inclination, position angle, and optical radius. The table notes note the subset of galaxies that that are close and large enough for us to carry out the multi-resolution analysis in Section 4.3. We adopt distances from Kennicutt et al. (2011) where possible and from Walter et al. (2008) elsewhere. We take orientations from Walter et al. 


\begin{tabular}{|c|c|c|c|c|c|c|c|}
\hline Galaxy & $\begin{array}{c}D \\
(\mathrm{Mpc})\end{array}$ & $\begin{array}{l}\text { Res. } \\
(\mathrm{kpc})\end{array}$ & $\begin{array}{c}i \\
\left(^{\circ}\right)\end{array}$ & $\begin{array}{l}\text { P.A. } \\
\left({ }^{\circ}\right)\end{array}$ & $\begin{array}{l}r_{25} \\
\left({ }^{\prime}\right)\end{array}$ & $\begin{array}{c}r_{25} \\
(\mathrm{kpc})\end{array}$ & Multiscale \\
\hline (1) & (2) & (3) & (4) & (5) & (6) & (7) & (8) \\
\hline NGC 0337 & $19.3^{\mathrm{K}}$ & $1.24^{\mathrm{a}}$ & 51 & 90 & 1.5 & 10.6 & $\ldots$ \\
\hline NGC 0628 & $7.2^{\mathrm{K}}$ & 0.46 & 7 & 20 & 4.9 & 10.4 & $\checkmark$ \\
\hline NGC 0925 & $9.1^{\mathrm{K}}$ & 0.59 & 66 & 287 & 5.4 & 14.3 & $\ldots$ \\
\hline NGC 2403 & $3.2^{\mathrm{W}}$ & 0.21 & 63 & 124 & 7.9 & 7.4 & $\checkmark$ \\
\hline NGC 2841 & $14.1^{\mathrm{K}}$ & 0.91 & 74 & 153 & 3.5 & 14.2 & $\ldots$ \\
\hline NGC 2903 & $8.9^{\mathrm{W}}$ & 0.57 & 65 & 204 & 5.9 & 15.2 & $\checkmark$ \\
\hline NGC 2976 & $3.6^{\mathrm{K}}$ & 0.23 & 65 & 335 & 3.6 & 3.8 & $\ldots$ \\
\hline NGC 3049 & $19.2^{\mathrm{K}}$ & $1.24^{\mathrm{a}}$ & 58 & 28 & 1.0 & 2.7 & $\ldots$ \\
\hline NGC 3184 & $11.8^{\mathrm{K}}$ & 0.76 & 16 & 179 & 3.7 & $\ldots$ & \\
\hline NGC 3198 & $14.1^{\mathrm{K}}$ & 0.91 & 72 & 215 & 3.2 & 13.0 & $\ldots$ \\
\hline NGC 3351 & $9.3^{\mathrm{K}}$ & 0.60 & 41 & 192 & 3.6 & 10.6 & $\ldots$ \\
\hline NGC 3521 & $11.2^{\mathrm{K}}$ & 0.72 & 73 & 340 & 4.2 & 12.9 & $\ldots$ \\
\hline NGC 3627 & $9.4^{\mathrm{K}}$ & 0.61 & 62 & 173 & 5.1 & 13.8 & $\checkmark$ \\
\hline NGC 3938 & $17.9^{\mathrm{K}}$ & $1.15^{\mathrm{a}}$ & 14 & 15 & 1.8 & 6.3 & $\ldots$ \\
\hline NGC 4214 & $2.9^{\mathrm{W}}$ & 0.19 & 44 & 65 & 3.4 & 2.9 & $\ldots$ \\
\hline NGC 4254 & $14.4^{\mathrm{K}}$ & 0.93 & 32 & 55 & 2.5 & 14.6 & $\ldots$ \\
\hline NGC 4321 & $14.3^{\mathrm{K}}$ & 0.92 & 30 & 153 & 3.0 & 12.5 & $\ldots$ \\
\hline NGC 4536 & $14.5^{\mathrm{K}}$ & 0.94 & 59 & 299 & 3.5 & 14.9 & $\ldots$ \\
\hline NGC 4559 & $7.0^{\mathrm{K}}$ & 0.45 & 65 & 328 & 5.2 & 10.7 & $\ldots$ \\
\hline NGC 4569 & $9.86^{\mathrm{K}}$ & 0.64 & 66 & 23 & 4.6 & 26.5 & $\ldots$ \\
\hline NGC 4579 & $16.4^{\mathrm{K}}$ & $1.06^{\mathrm{a}}$ & 39 & 100 & 2.5 & 15.0 & $\cdots$ \\
\hline NGC 4625 & $9.3^{\mathrm{K}}$ & 0.60 & 47 & 330 & 0.7 & 1.9 & $\ldots$ \\
\hline NGC 4725 & $11.9^{\mathrm{K}}$ & 0.77 & 54 & 36 & 4.9 & 13.2 & $\checkmark$ \\
\hline NGC 4736 & $4.7^{\mathrm{K}}$ & 0.30 & 41 & 296 & 3.9 & 5.3 & $\checkmark$ \\
\hline NGC 5055 & $7.9^{\mathrm{K}}$ & 0.51 & 59 & 102 & 5.9 & 17.3 & $\ldots$ \\
\hline NGC 5194 & $7.9^{\mathrm{W}}$ & 0.52 & 20 & 172 & 3.9 & 9.0 & $\checkmark$ \\
\hline NGC 5457 & $6.7^{\mathrm{K}}$ & 0.43 & 18 & 39 & 12.0 & 25.8 & $\checkmark$ \\
\hline NGC 5713 & $21.4^{\mathrm{K}}$ & $1.38^{\mathrm{a}}$ & 48 & 11 & 1.2 & 9.5 & $\ldots$ \\
\hline NGC 6946 & $6.8^{\mathrm{K}}$ & 0.44 & 33 & 243 & 5.7 & 9.8 & $\checkmark$ \\
\hline NGC 7331 & $14.5^{\mathrm{K}}$ & 0.94 & 76 & 168 & 4.6 & 19.5 & $\ldots$ \\
\hline
\end{tabular}

Notes. Sample used in this paper. Columns give (1) galaxy name; (2) adopted distance in Mpc; (3) FWHM spatial resolution of HERACLES data at that distance, in kiloparsecs; (4) adopted inclination; and (5) position angle in degrees; adopted radius of the $B$-band 25 th magnitude isophote, used to normalize the radius in (6) arcminutes and (7) kiloparsecs. Most analysis in this paper considers data inside $0.75 r_{25}$. Column 8 indicates if the galaxy is close and large enough for the multiscale analysis in Section 4.3.

a Too distant to convolve to $1 \mathrm{kpc}$ resolution. Included in analysis at native resolution.

K,W Distance adopted from K: Kennicutt et al. (2011) or W: Walter et al. (2008).

(2008) and from LEDA (Prugniel \& Heraudeau 1998) and NED elsewhere.

Table 2 reports integrated and disk-average properties for our sample. We report our integrated stellar mass estimate, galaxy morphology, metallicity and dust-to-gas ratio at $\approx 0.4 r_{25}$, average gas mass and SFR surface density inside $0.75 r_{25}$, our parameterized rotation curve fit, and the orbital time at $0.4 r_{25}$. We take metallicities from Moustakas et al. (2010), averaging their PT05 and KK04 strong-line calibrations. They argue that these two calibrations bracket the true metallicity and that the relative ordering of metallicities is robust (see also Kewley \& Ellison 2008), but the uncertainty in the absolute value is considerable. For cases where Moustakas et al. (2010) do not present a metallicity, we draw one from the recent compilations by Marble et al. (2010) and Calzetti et al. (2010).

\subsection{Methodology}

We sample our targets at $1 \mathrm{kpc}$ resolution. This is fine enough to isolate many key physical conditions in the ISM: metallicity, coarse kinematics, gas and stellar surface density. At the same time, we expect to average several star-forming regions in each element (e.g., Schruba et al. 2010), with $M_{\text {mol }} \gtrsim 10^{7} M_{\odot}$ and $M_{*} \gtrsim 10^{4} M_{\odot}$ formed over the last $\sim 5 \mathrm{Myr}$ in each element. This minimizes concerns about evolution of individual regions, sampling the initial mass function (IMF), and drift of stars or leakage of ionizing photons from their parent region.

We convolve each map to have a symmetric Gaussian beam with FWHM $1 \mathrm{kpc}$. For the Spitzer $24 \mu \mathrm{m}$ maps we first convert from the MIPS point-spread function to a $13^{\prime \prime}$ Gaussian beam using a kernel kindly provided by K. Gordon, and then we convolve to $1 \mathrm{kpc}$. This exercise effectively places our targets at a common distance but does not account for foreshortening along the minor axis. Five galaxies are too distant to convolve to $1 \mathrm{kpc}$. We mark these in Table 1 and include them in our analysis at their native resolution.

We sample each map to generate a set of intensity measurements. The sampling points are distributed on a hexagonal grid with points spaced by $0.5 \mathrm{kpc}$, one half-resolution element. At each sampling point we measure $\mathrm{CO}(2-1)$ intensity, $\mathrm{H}$ I intensity, a suite of SFR tracers (described in L12), dust properties, and $\Sigma_{*}$. We use these to estimate physical conditions as described above and in L12, taking into account the inclination of the galaxy.

We also identify a sample of galaxies to study the effects of physical resolution. Nine galaxies, marked in Table 1, have both the proximity and extent to allow us to test the effect of physical resolution on our results. We convolve these to a succession of physical resolutions from 0.6 to $2.4 \mathrm{kpc}$ for further analysis (Section 4.3).

We treat regions with $\Sigma_{\mathrm{SFR}}<10^{-3} M_{\odot} \mathrm{yr}^{-1} \mathrm{kpc}^{-2}$ or $I_{\mathrm{CO}}<2.5 \times \sigma_{\mathrm{CO}}$ as upper limits and consider only points with $r_{\text {gal }}<0.75 r_{25}$ - the HERACLES maps contain signal outside this radius (Schruba et al. 2011) but mostly not significant emission over individual lines of sight. In total we have $\sim 14,500$ lines of sight with at least one significant measurement, of which 1900 have CO upper limits and 1650 have SF upper limits. Points for which neither measurement is significant are not considered in the analysis. Nyquist sampling the maps in a hexagonal pattern leads to oversampling by a factor of $\sim 5$, so that this corresponds to $>2000$ independent measurements. The maximum $(2.5 \sigma)$ upper limit on $\Sigma_{\text {mol }}$ is $\approx 6 M_{\odot} \mathrm{pc}^{-2}$; the median upper limit is $\approx 2.6 M_{\odot} \mathrm{pc}^{-2}$.

\subsection{Literature Data}

We compare our results to recent measurements of SFR and molecular gas. These employ a variety of sampling schemes and SFR tracers. We adjust each to match our adopted CO-to$\mathrm{H}_{2}$ conversion factor and IMF. Contrasting our approach with these data illuminates the impact of methodology and allows us to explore whether diverse observations yield consistent results under matched assumptions.

Kennicutt (1998b) presented disk-averaged measurements for 57 normal spiral galaxies and 15 starburst galaxies. He used literature $\mathrm{CO}$ with a fixed $\alpha_{\mathrm{CO}}$ to estimate $\Sigma_{\text {mol }}$. To estimate $\Sigma_{\mathrm{SFR}}$, he used $\mathrm{H} \alpha$ in disk galaxies and IR emission in starbursts.

Calzetti et al. (2010) estimated disk-averaged $\Sigma_{\text {SFR }}$ for a large set of nearby galaxies. We cross-index these with integrated CO fluxes from Young et al. (1995), Helfer et al. (2003), and Leroy et al. (2009) to derive $\Sigma_{\mathrm{mol}}$ assuming that $\mathrm{CO}$ emission covers the same area as $\mathrm{H} \alpha$. From the combination of these data we have disk-average $\Sigma_{\mathrm{SFR}}$ and $\Sigma_{\text {mol }}$ estimates for 41 galaxies. 
Table 2

Sample Properties

\begin{tabular}{|c|c|c|c|c|c|c|c|c|c|}
\hline (1) & $\begin{array}{c}\log \left(M_{*}\right) \\
\log _{10}\left(M_{\odot}\right) \\
(2)\end{array}$ & $\begin{array}{l}\text { Morphology } \\
\text { T-Type } \\
\text { (3) }\end{array}$ & $\begin{array}{c}z \\
{[12+\log [\mathrm{O} / \mathrm{H}]]} \\
(4)\end{array}$ & $\begin{array}{l}\mathrm{D} / \mathrm{G} \\
(5)\end{array}$ & $\begin{array}{c}\left\langle\Sigma_{\mathrm{H} \mathrm{I}+\mathrm{H} 2}\right\rangle \\
\left(M_{\odot} \mathrm{pc}^{-2}\right) \\
(6)\end{array}$ & $\begin{array}{c}\left\langle\Sigma_{\mathrm{SFR}}\right\rangle \\
\left(10^{-3} M_{\odot} \mathrm{yr}^{-1} \mathrm{kpc}^{-2}\right) \\
(7)\end{array}$ & $\begin{array}{c}v_{\text {flat }} \\
\left(\mathrm{km} \mathrm{s}^{-1}\right) \\
(8)\end{array}$ & $\begin{array}{c}l_{\text {flat }} \\
(\mathrm{kpc}) \\
(9)\end{array}$ & $\begin{array}{c}\left\langle\tau_{\text {orb }}\right\rangle \\
\left(10^{8} \mathrm{yr}\right) \\
(10)\end{array}$ \\
\hline NGC 0337 & 9.9 & 6.7 & 8.51 & 0.004 & 21 & 14 & 130 & 2.9 & 2.4 \\
\hline NGC 0628 & 10.0 & 5.2 & 8.69 & 0.012 & 15 & 4.0 & 200 & 0.8 & 1.3 \\
\hline NGC 0925 & 9.7 & 7.0 & 8.52 & 0.004 & 7.5 & 1.3 & 140 & 6.9 & 4.4 \\
\hline NGC 2403 & 9.6 & 6.0 & 8.57 & 0.009 & 10 & 3.3 & 120 & 0.95 & 1.6 \\
\hline NGC 2841 & 10.7 & 3.0 & 8.88 & 0.037 & 4.6 & 1.4 & 310 & 2.3 & 1.2 \\
\hline NGC 2903 & 10.4 & 4.0 & $8.90^{\mathrm{a}}$ & 0.012 & 12 & 5.7 & 210 & 2.4 & 2.0 \\
\hline NGC 2976 & 9.0 & 5.2 & 8.67 & 0.008 & 7.6 & 4.4 & 88 & 1.1 & 1.4 \\
\hline NGC 3049 & 9.5 & 2.5 & 8.82 & 0.005 & 8.0 & 10 & 180 & 3.0 & 1.5 \\
\hline NGC 3184 & 10.2 & 6.0 & 8.83 & 0.018 & 14 & 2.8 & 200 & 2.5 & 1.8 \\
\hline NGC 3198 & 10.0 & 5.2 & 8.62 & 0.012 & 8.4 & 2.3 & 150 & 3.0 & 2.6 \\
\hline NGC 3351 & 10.1 & 3.1 & 8.90 & 0.018 & 8.5 & 5.2 & 200 & 1.1 & 1.2 \\
\hline NGC 3521 & 10.7 & 4.0 & 8.70 & 0.012 & 22 & 7.8 & 229 & 1.5 & 1.5 \\
\hline NGC 3627 & 10.5 & 3.1 & 8.67 & 0.016 & 13 & 7.7 & 190 & 1.1 & 1.8 \\
\hline NGC 3938 & 10.3 & 5.1 & $8.71^{\mathrm{a}}$ & 0.018 & 22 & 7.9 & 140 & 0.73 & 1.6 \\
\hline NGC 4214 & 8.7 & 9.8 & $8.36^{\mathrm{a}}$ & 0.0038 & 9.2 & 8.4 & 350 & 11 & 2.0 \\
\hline NGC 4254 & 10.5 & 5.2 & 8.79 & 0.01 & 47 & 18 & 170 & 1.4 & 1.6 \\
\hline NGC 4321 & 10.6 & 4.1 & 8.84 & 0.012 & 30 & 9.0 & 229 & 1.8 & 1.4 \\
\hline NGC 4536 & 10.2 & 4.3 & 8.61 & 0.005 & 13 & 6.8 & 180 & 0.7 & 2.0 \\
\hline NGC 4559 & 9.5 & 6.0 & 8.55 & 0.005 & 10 & 1.8 & 100 & 2.1 & 2.9 \\
\hline NGC 4569 & 10.2 & 2.4 & $8.88^{a}$ & 0.017 & 8.5 & 1.9 & 220 & 3.2 & 1.8 \\
\hline NGC 4579 & 10.7 & 2.8 & $8.93^{\mathrm{a}}$ & 0.021 & 13 & 3.8 & 270 & 1.7 & 1.2 \\
\hline NGC 4625 & 8.9 & 8.8 & 8.70 & 0.011 & 8.5 & 6.6 & 27 & 0.53 & 2.3 \\
\hline NGC 4725 & 10.5 & 2.2 & 8.73 & 0.03 & 4.5 & 0.75 & 220 & 1.1 & 1.9 \\
\hline NGC 4736 & 10.2 & 2.4 & 8.66 & 0.008 & 17 & 10 & 170 & 0.25 & 0.77 \\
\hline NGC 5055 & 10.5 & 4.0 & 8.77 & 0.02 & 18 & 4.1 & 200 & 0.71 & 1.7 \\
\hline NGC 5194 & 10.5 & 4.0 & 8.87 & 0.02 & 53 & 20 & 210 & 0.58 & 1.0 \\
\hline NGC 5457 & 10.4 & 6.0 & $8.46^{\mathrm{a}}$ & 0.013 & 10 & 2.4 & 210 & 1.2 & 2.7 \\
\hline NGC 5713 & 10.3 & 4.0 & 8.64 & 0.006 & 54 & 37 & $\ldots$ & $\ldots$ & $\ldots$ \\
\hline NGC 6946 & 10.5 & 5.9 & 8.73 & 0.007 & 37 & 21 & 190 & 1.2 & 1.5 \\
\hline NGC 7331 & 10.8 & 3.9 & 8.68 & 0.01 & 16 & 4.4 & 260 & 1.9 & 1.9 \\
\hline
\end{tabular}

Notes. Properties of sample galaxies. Columns give (1) galaxy name; (2) integrated stellar mass of whole galaxies based on $3.6 \mu \mathrm{m}$ flux of Dale et al. (2007, 2009); (3) morphology; (4) "characteristic" metallicity at 0.4r 25 from Moustakas et al. (2010), averaging their PT05 and KK04 calibrations; (5) dust-to-gas ratio at $0.4 r_{25}$ based on our modeling of Spitzer data; (6) average $\mathrm{H}_{\mathrm{I}}+\mathrm{H}_{2}$ surface density inside $0.75 r_{25}$; (7) average SFR surface density inside $0.75 r_{25}$; parameters for simple rotation curve fit, (8) $v_{\text {flat }}$ and (9) $l_{\text {flat }}$; and (10) orbital time at $0.4 r_{25}$ based on the rotation curve.

${ }^{a}$ Metallicity from compilation of Calzetti et al. (2010) and Marble et al. (2010) or Kennicutt et al. (2003b) (NGC 5457).

Saintonge et al. (2012), following Saintonge et al. (2011), present the COLDGASS survey, which obtained integrated molecular gas mass and SFRs for 366 galaxies with $M_{*}>$ $10^{10} M_{\odot}, 215$ with secure $\mathrm{CO}$ detections. This large survey represents the best sample of integrated galaxy measurements to date. To convert to surface densities, we take the area of the star-forming disk in these galaxies to be $0.75 r_{25}$. Saintonge et al. (2012) derive their SFRs from SED modeling that yields results close to what one would obtain converting the UV+IR luminosity directly to an SFR. This yields higher SFRs than our approach for matched measurements. Comparing galaxies with matched stellar mass or molecular gas content, we find the offset to be $\approx 0.19 \mathrm{dex}$, a factor of $\approx 1.55$. This agrees well with what one would expect accounting for our subtraction of an IR cirrus that accounts for $\approx 20 \%$ of the total emission and our $24 \mu \mathrm{m}$ coefficient, which is $\approx 1.2$ lower than what one would adopt to match a bolometric total infrared SFR indicator (see L12 for calculations and discussion).

Leroy et al. (2005) combined new data with measurements by Young et al. (1995), Elfhag et al. (1996), Taylor et al. (1998), Böker et al. (2003), and Murgia et al. (2002) to compare $\Sigma_{\mathrm{SFR}}$ and $\Sigma_{\mathrm{mol}}$ for individual $\sim 30^{\prime \prime}-50^{\prime \prime}$ pointings in a wide sample of nearby galaxies. They estimate $\Sigma_{\text {SFR }}$ from the $20 \mathrm{~cm}$ radio continuum (Condon 1992). These low-resolution pointings typically cover several $\mathrm{kpc}$, a larger area than our resolution elements but less than an average over a whole galaxy disk.

Wong \& Blitz (2002), Schuster et al. (2007), and Crosthwaite $\&$ Turner (2007) presented radial profiles of $\Sigma_{\text {mol }}$ and $\Sigma_{\text {SFR }}$ for several nearby galaxies. Wong \& Blitz (2002) targeted seven nearby spirals, using $\mathrm{H} \alpha$ to calculate $\Sigma_{\mathrm{SFR}}$. Schuster et al. (2007) targeted M51 and derived $\Sigma_{\mathrm{SFR}}$ from $20 \mathrm{~cm}$ radio continuum to estimate $\Sigma_{\mathrm{SFR}}$. We only present the Wong \& Blitz (2002) and Schuster et al. (2007) profiles down to $\Sigma_{\mathrm{mol}} \approx 5 \mathrm{M}_{\odot} \mathrm{pc}^{-2}$, below which we consider them somewhat unreliable. Crosthwaite \& Turner (2007) targeted NGC 6946 and used IR emission to estimate $\Sigma_{\mathrm{SFR}}$.

Kennicutt et al. (2007), Blanc et al. (2009), Rahman et al. (2011), and Rahman et al. (2012) targeted small regions, similar to B08 and the work presented here. Kennicutt et al. (2007) focused on luminous regions in M51, mainly in the spiral arms. They infer $\Sigma_{\text {SFR }}$ from a combination of $\mathrm{H} \alpha$ and $24 \mu \mathrm{m}$ emission. Rahman et al. (2011) explored a range of methodologies. We focus on their most robust measurements, drawn from bright 
regions in NGC 4254 with $\Sigma_{\text {SFR }}$ from a combination of NUV and $24 \mu \mathrm{m}$ emission. Rahman et al. (2012) extended this work to consider the full set of CARMA STING galaxies, using only the $24 \mu \mathrm{m}$ emission with a nonlinear calibration to infer $\Sigma_{\mathrm{SFR}}$. Blanc et al. (2009) studied the central $4.1 \times 4.1 \mathrm{kpc}^{2}$ of M51, deriving $\Sigma_{\mathrm{SFR}}$ from $\mathrm{H} \alpha$ spectroscopy corrected using the Balmer decrement.

\section{3. $\Sigma_{\mathrm{SFR}}-\Sigma_{\mathrm{mol}}$ SCALING RELATIONS: FIRST-ORDER CONSTANCY OF $\tau_{\mathrm{dep}}^{\mathrm{mol}}$}

We estimate $\Sigma_{\text {SFR }}$ and $\Sigma_{\text {mol }}$ for $\sim 14,500$ points in 30 nearby galaxies. In this section we analyze these data in the context of a traditional "star formation law" scaling relation (Section 1.1). We show the data distribution in $\Sigma_{\mathrm{SFR}}-\Sigma_{\text {mol }}$ parameter space (Section 3.1) and examine how this depends on methodology (Section 3.2). Using a Monte Carlo technique based on that of Blanc et al. (2009), we consider the best-fit power-law to the ensemble data and individual galaxies (Section 3.3). We compare our results to a broad sample of literature data (Section 3.4).

\subsection{Combined Measurement}

Figure 1 compares $\Sigma_{\mathrm{SFR}}$, estimated from $\mathrm{H} \alpha+24 \mu \mathrm{m}$, and $\Sigma_{\text {mol }}$ at $1 \mathrm{kpc}$ resolution for our whole sample. Individual kpc resolution lines of sight appear as gray points, and the red points show the median $\Sigma_{\mathrm{SFR}}$ and standard deviation after binning the data by $\Sigma_{\mathrm{mol}}$. In the top right panel and bottom row, blue contours show data density adopting different weightings. The top right panel gives identical weight to each line of sight, treating each $\mathrm{kpc}^{2}$ as equal regardless of location. The bottom left panel gives equal weight to each galaxy and so weights measurements from small galaxies with little area more than measurements from large galaxies. The bottom right panel treats each radial ring in each galaxy equally, and so gives more weight to the central parts of galaxies than their outer regions. Dashed lines here and throughout this paper indicate fixed $\tau_{\text {dep }}^{\text {mol }}$, and a horizontal line indicates the limit of our $\Sigma_{\mathrm{SFR}}$ measurements. In the top left panel, dark points show measurements where one quantity is an upper limit. Table 3 summarizes key values from the plots in this section.

The top rows of Figure 1 and Table 3 show the good correspondence between $\Sigma_{\mathrm{SFR}}$ and $\Sigma_{\text {mol }}$ that we have previously found in the HERACLES sample (B08; L08; Schruba et al. 2011; Bigiel et al. 2011). Our dynamic range at $1 \mathrm{kpc}$ resolution spans from $\Sigma_{\mathrm{SFR}} \sim 10^{-3}$ to $10^{-1} M_{\odot} \mathrm{yr}^{-1} \mathrm{kpc}^{-2}$ and $\Sigma_{\text {mol }}$ from a few to $100 M_{\odot} \mathrm{pc}^{-2}$. Across this range, $\Sigma_{\mathrm{mol}}$ and $\Sigma_{\mathrm{SFR}}$ correlate well, exhibiting a Spearman rank correlation coefficient $\gtrsim 0.7$ for most tracers and weightings. This quantifies the tight, oneto-one relationship visible by eye in the top row.

The median $\tau_{\text {dep }}^{\text {mol }}$ weighting each line of sight equally is 2.2 Gyr with a scatter of 0.3 dex, a factor of two. The absolute value of the median $\tau_{\mathrm{dep}}^{\mathrm{mol}}$, i.e., the scale of the $x$ - and $y$-axes in Figure 1, depends on the calibration of our SFR tracer and $\mathrm{CO}-$ to $-\mathrm{H}_{2}$ conversion factor. Each remains uncertain at the $30 \%-50 \%$ level, and we suggest that an overall uncertainty

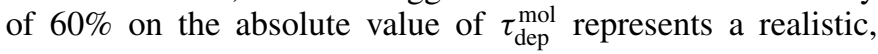
if somewhat conservative, value. Our $\Sigma_{\mathrm{mol}}$ and $\Sigma_{\mathrm{SFR}}$ estimates can be compared internally with much better accuracy than this (L12; Sandstrom et al. 2012), so we suggest that this uncertainty be viewed as an overall scaling of our results.

The bottom row in Figure 1 begins to reveal the deviations from a simple one-to-one scaling that will be the subject of
Table 3

$\tau_{\text {dep }}^{\text {mol }}$ at $1 \mathrm{kpc}$ Resolution

\begin{tabular}{lccc}
\hline \hline Tracer & $\begin{array}{c}\text { Median } \tau_{\mathrm{dep}}^{\text {mol }} \\
(\mathrm{Gyr})\end{array}$ & $\begin{array}{c}\text { Scatter } \\
(1 \sigma \text { dex })\end{array}$ & $\begin{array}{c}r_{\text {corr }} \\
\left(\Sigma_{\text {mol }}, \Sigma_{\mathrm{SFR}}\right)\end{array}$ \\
\hline \multicolumn{3}{c}{ Weighting as equal each $\ldots$} \\
\hline Line of sight & & \\
$\ldots$ fixed $\alpha_{\mathrm{CO}}$ & 2.2 & 0.28 & $0.72 \pm 0.02$ \\
$\ldots \Sigma=100 \alpha_{\mathrm{CO}}$ & 2.6 & 0.26 & $0.75 \pm 0.01$ \\
$\ldots \Sigma=50 \alpha_{\mathrm{CO}}$ & 3.1 & 0.28 & $0.70 \pm 0.01$ \\
Galaxy & & & \\
$\ldots$ fixed $\alpha_{\mathrm{CO}}$ & 1.3 & 0.32 & $0.67 \pm 0.19$ \\
$\ldots \ldots$ only $M_{*}>10^{10} M_{\odot}$ & 1.7 & 0.21 & $0.87 \pm 0.18$ \\
$\ldots \ldots$ only $M_{*}<10^{10} M_{\odot}$ & 0.4 & 0.29 & $0.53 \pm 0.37$ \\
$\ldots \Sigma=100 \alpha_{\mathrm{CO}}$ & 1.8 & 0.20 & $0.89 \pm 0.17$ \\
$\ldots \ldots$ only $M_{*}>10^{10} M_{\odot}$ & 2.0 & 0.13 & $0.95 \pm 0.25$ \\
$\ldots \ldots$ only $M_{*}<10^{10} M_{\odot}$ & 1.1 & 0.26 & $0.87 \pm 0.35$ \\
$\ldots \Sigma=50 \alpha_{\mathrm{CO}}$ & 2.4 & 0.26 & $0.90 \pm 0.19$ \\
$\ldots \ldots$ only $M_{*}>10^{10} M_{\odot}$ & 2.1 & 0.31 & $0.94 \pm 0.21$ \\
$\ldots \ldots$ only $M_{*}<10^{10} M_{\odot}$ & 2.7 & 0.21 & $0.78 \pm 0.44$ \\
\hline
\end{tabular}

Tracing $\Sigma_{\text {SFR }}$ with ...

(weighting lines of sight equally)

\begin{tabular}{llll}
\hline $\mathrm{H} \alpha+24 \mu \mathrm{m}$ & & & \\
$\ldots$ best estimate & 2.2 & 0.28 & $0.72 \pm 0.02$ \\
$\ldots$ no cirrus & 2.0 & 0.22 & $0.79 \pm 0.02$ \\
$\ldots$ double cirrus & 3.0 & 0.37 & $0.62 \pm 0.02$ \\
FUV $+24 \mu \mathrm{m}$ & & & \\
$\ldots$ best estimate & 2.2 & 0.27 & $0.72 \pm 0.02$ \\
$\ldots$ no cirrus & 1.9 & 0.21 & $0.81 \pm 0.02$ \\
$\ldots$ double cirrus & 3.2 & 0.39 & $0.58 \pm 0.02$ \\
$\mathrm{H} \alpha+1$ mag & 2.1 & 0.30 & $0.66 \pm 0.02$ \\
\hline
\end{tabular}

Notes. Median molecular gas depletion time, scatter, and correlation between $\Sigma_{\mathrm{SFR}}$ and $\Sigma_{\mathrm{mo}}$ in our sample. Line-of-sight averages treat each kpc-resolution line of sight as equal. Galaxy averages refer to $\tau_{\text {dep }}^{\text {mol }}=\left\langle\Sigma_{\text {mol }}\right\rangle /\left\langle\Sigma_{\mathrm{SFR}}\right\rangle$ inside $0.75 r_{25}$ for each galaxy. Unless otherwise noted, we calculate $\Sigma_{\text {mol }}$ using fixed $\alpha_{\mathrm{CO}}$ and $\Sigma_{\mathrm{SFR}}$ from $\mathrm{H} \alpha+24 \mu \mathrm{m}$. Quoted error bars on $\tau_{\mathrm{dep}}^{\mathrm{mol}}$ report $1 \sigma$ scatter; uncertainties on the rank correlation arise from randomly repairing data.

Section 4. Weighting all galaxies equally (bottom left panel) reveals a significant population of low $\Sigma_{\text {mol }}$, high $\Sigma_{\mathrm{SFR}}$, low $\tau_{\mathrm{dep}}^{\mathrm{mol}}$ data. This drives the median depletion time for the sample from $\approx 2.2 \mathrm{Gyr}$, weighting by line of sight, to $\approx 1.3 \mathrm{Gyr}$, weighting by galaxy. In Section 4.1 we show that these low apparent $\tau_{\text {dep }}^{\text {mol }}$ originate from low-mass, low-metallicity systems (see also Schruba et al. 2011; Krumholz et al. 2011; Schruba et al. 2012). Because of their small size, these systems do not contribute many data compared to large, metal-rich spirals. Therefore, they only weakly influence the overall data distribution seen in the top row. We examine $\tau_{\mathrm{dep}}^{\mathrm{mol}}$ as a function of host galaxy properties and local conditions in Sections 4.1 and 4.2. In Appendix D we present $\Sigma_{\mathrm{SFR}}-\Sigma_{\text {mol }}$ relations for individual galaxies (see also Table 2), allowing the reader to see how Figure 1 emerges from the superposition of individual systems (see also Section 3.3).

Weighting radial rings equally (bottom right panel) highlights these same low $\tau_{\text {dep }}^{\text {mol }}$, low $\Sigma_{\text {mol }}$ galaxies and brings out an additional low $\tau_{\mathrm{dep}}^{\mathrm{mol}}$ population at higher $\Sigma_{\mathrm{mol}}$. These points emerge because the radial weighting emphasizes points in the central parts of galaxies relative to their outskirts. We show in Section 4.4 that the central regions of many of our targets exhibit enhanced efficiency compare to their disks. As will small galaxies, these central regions contribute only a tiny fraction of the area in our survey and thus exert little impact on the plots in the top row. 


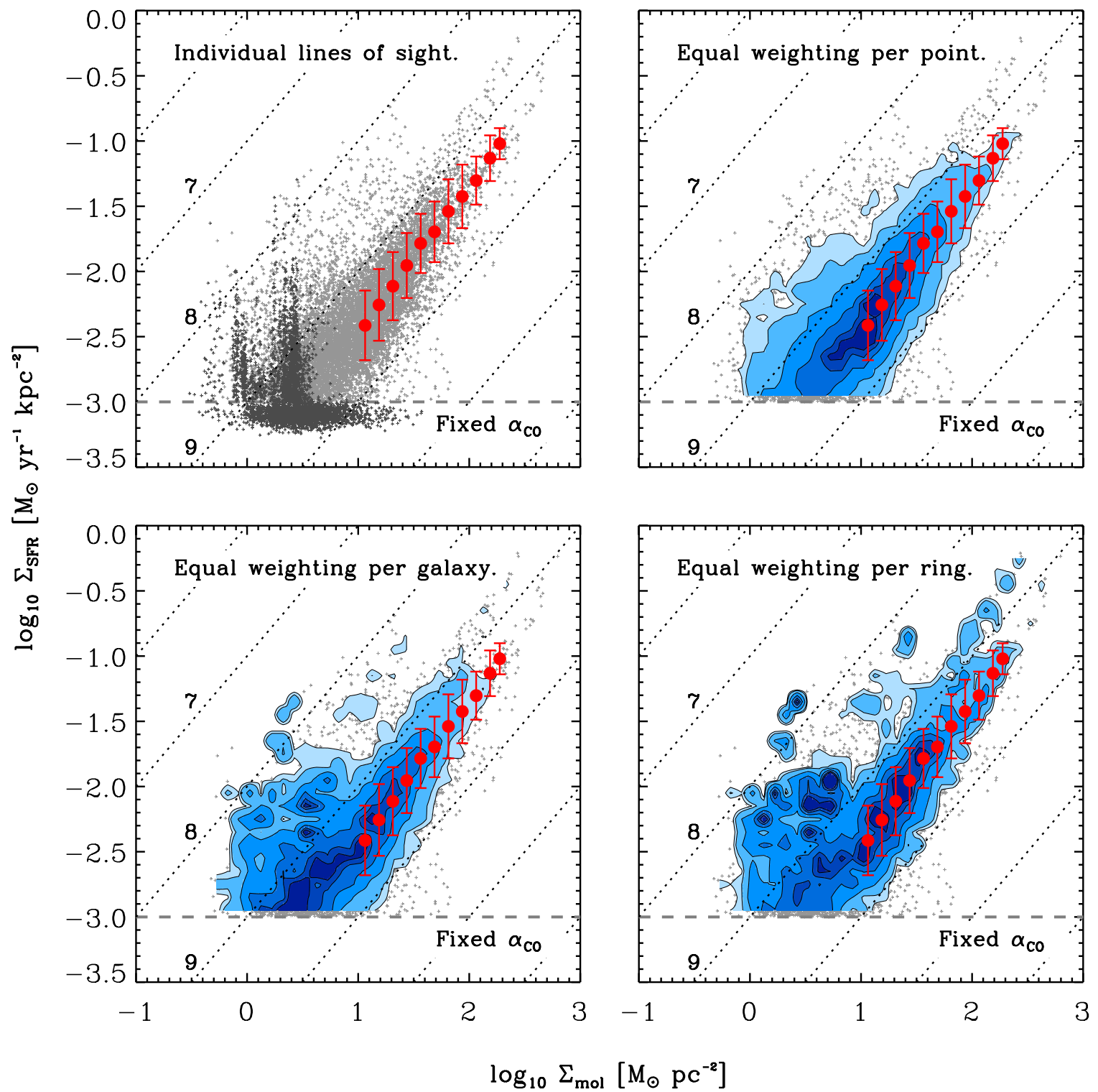

Figure 1. Star formation rate surface density, $\Sigma_{\mathrm{SFR}}$, estimated from $\mathrm{H} \alpha+24 \mu \mathrm{m}$ emission, as a function of molecular gas surface density, $\Sigma_{\mathrm{mol}}$, derived from CO (2-1) emission for 30 nearby disk galaxies. The top left panel shows individual points (dark gray points show upper limits) with the running median and standard deviation indicated by red points and error bars. The red points with error bars from the first panel appear in all four panels to allow easy comparison. Dotted lines indicated fixed $\mathrm{H}_{2}$ depletion times; the number indicates $\log _{10} \tau_{\text {Dep }}$ in yr. The top right panel shows the density of the data in the top left panel. In the bottom panels we vary the weighting used to derive data density. The bottom left panel gives equal weight to each galaxy. The bottom right panel gives equal weight to each galaxy and each radial bin.

Figure 1 thus illustrates our main conclusions: a first order simple linear correlation between $\Sigma_{\mathrm{SFR}}$ and $\Sigma_{\text {mol }}$ and real secondorder variations. It also illustrates the limitation of considering only $\Sigma_{\mathrm{SFR}}-\Sigma_{\text {mol }}$ parameter space to elicit these second-order variations. Metallicity, dust-to-gas ratio, and position in a galaxy all play key roles but are not encoded in this plot, leading to double-valued $\Sigma_{\mathrm{SFR}}$ at fixed $\Sigma_{\mathrm{mol}}$ in some regimes. We explore these systematic variations in $\tau_{\text {dep }}^{\text {mol }}$ and motivate our explanations throughout the rest of the paper.

\subsection{Relationship for Different SFR and Molecular Gas Tracers}

Figure 1 shows our best-estimate $\Sigma_{\mathrm{SFR}}$ and $\Sigma_{\text {mol }}$ computed from fixed $\alpha_{\mathrm{CO}}$. Many approaches exist to estimate each quantity (see references in Leroy et al. 2011, L12), and the recent literature includes many claims about the effect of physical parameter estimation on the relation between $\Sigma_{\mathrm{SFR}}$ and $\Sigma_{\text {mol }}$. In this section, we explore the effects of varying our approach to estimate $\Sigma_{\mathrm{SFR}}$ and $\Sigma_{\mathrm{mol}}$.

\subsubsection{Choice of SFR Tracer}

Figure 2 and the lower part of Table 3 report the results of varying our approach to trace the SFR. We show $\Sigma_{\mathrm{SFR}}$ estimated from only $\mathrm{H} \alpha$, with a fixed, typical $A_{\mathrm{H} \alpha}=1 \mathrm{mag}$ (top left), along with results combining FUV, instead of $\mathrm{H} \alpha$, with $24 \mu \mathrm{m}$ emission (top right). We also show the results of varying the approach to the IR cirrus. Our best-estimate $\Sigma_{\text {SFR }}$ combines $\mathrm{H} \alpha$ or FUV with $24 \mu \mathrm{m}$ after correcting the $24 \mu \mathrm{m}$ emission for contamination by an IR cirrus following L12. We illustrate the impact of this correction by plotting results for two limiting cases of IR cirrus correction: no cirrus subtraction (bottom left) and removing double our best cirrus estimate (bottom right), which we consider a maximum reasonable correction. 


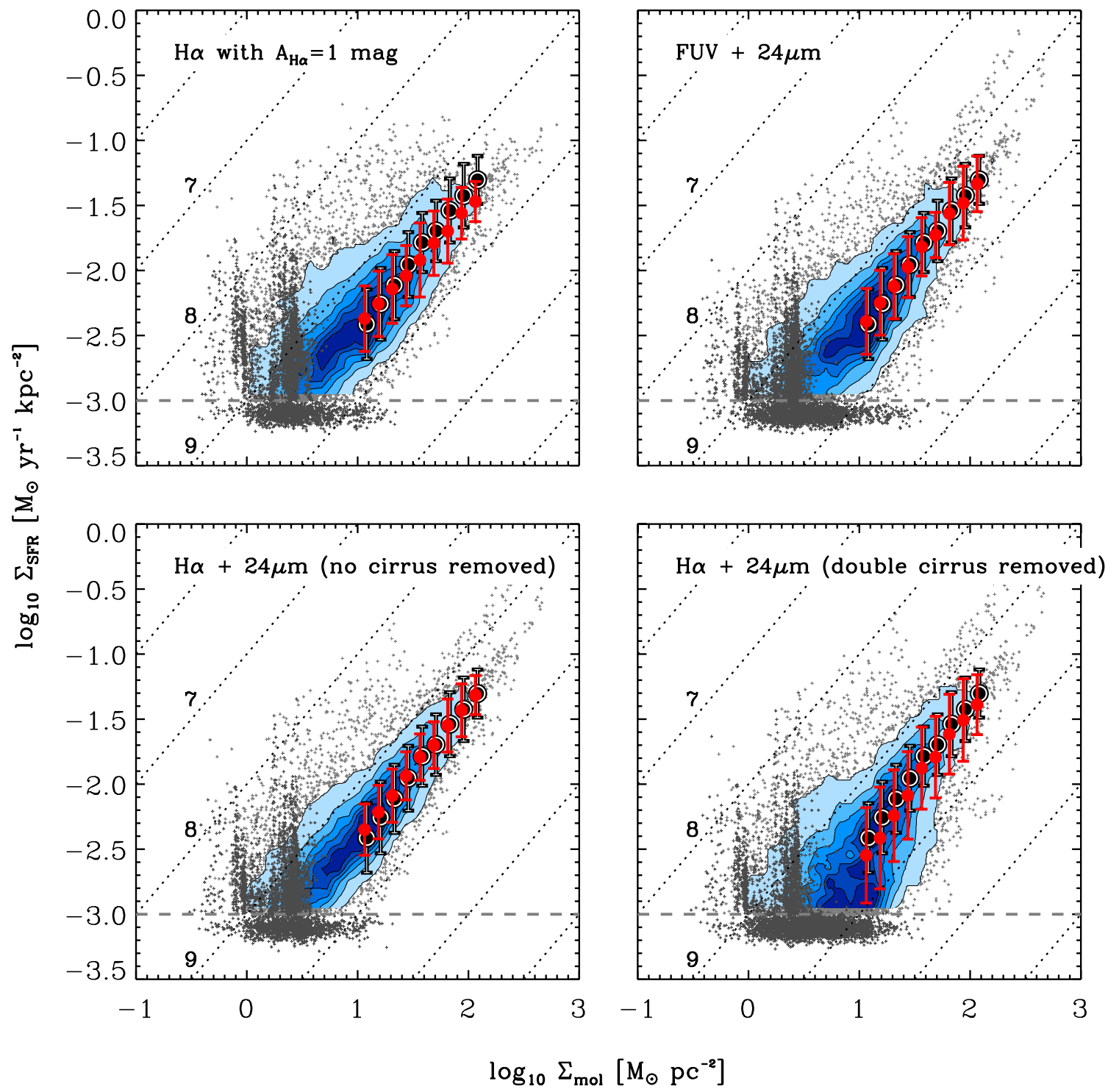

Figure 2. Star formation rate surface density, $\Sigma_{\mathrm{SFR}}$, estimated using different tracers as a function of molecular gas surface density, $\Sigma_{\text {mol }}$. Light gray points show individual measurements, dark gray points indicate upper limits, contours show data density, and red points show the running median and standard deviation using each tracer. For comparison, black-and-white points show the bins from the top right panel of Figure 1 . We show $\Sigma_{\mathrm{SFR}}$ estimated from H $\alpha$ assuming 1 magnitude of extinction (top left), a combination of FUV and $24 \mu \mathrm{m}$ emission (top right; L12), $\mathrm{H} \alpha$ combined with $24 \mu \mathrm{m}$ emission with no IR cirrus correction applied to the $24 \mu \mathrm{m}$ (bottom left), and $\mathrm{H} \alpha$ combined with $24 \mu \mathrm{m}$ with double our IR cirrus correction applied to the $24 \mu \mathrm{m}$ (bottom right).

Data density contours in Figure 2 weight each point equally, and the large black points indicate the original binned results from Figure 1.

The top left panel of Figure 2 and Table 3 show that the basic relationship between $\Sigma_{\mathrm{SFR}}$ and $\Sigma_{\text {mol }}$ persists even when we derive $\Sigma_{\text {SFR }}$ from $\mathrm{H} \alpha$ alone. The median $\tau_{\text {dep }}^{\text {mol }}$ and scatter using only $\mathrm{H} \alpha$ resemble what we find for our best estimate, and the correlation between $\mathrm{H} \alpha$ and $\mathrm{CO}$ appears only moderately weaker than for the hybrid SFR tracer. It also appears moderately flatter than relations that incorporate IR emission as we underestimate extinction in the central parts of galaxies (Section 3.3). Inasmuch as $\mathrm{H} \alpha$ represents an unambiguous tracer of recent star formation, the top left panel in Figure 2 demonstrates that subtle biases in the treatment of IR emission, e.g., $24 \mu \mathrm{m}$ emission tracing the ISM rather than recent star formation, do not drive our results.

The top right panel shows $\Sigma_{\mathrm{SFR}}$ traced by FUV $+24 \mu \mathrm{m}$ emission. The distribution agrees well with what we found using
$\mathrm{H} \alpha+24 \mu \mathrm{m}$, as do the median and scatter in $\tau_{\mathrm{dep}}^{\mathrm{mol}}$. The agreement of FUV+24 $\mu \mathrm{m}$ and $\mathrm{H} \alpha$ with our best estimate $\mathrm{H} \alpha+24 \mu \mathrm{m}$ occurs partially because we have designed our SFR tracers to yield self-consistent results (L12). However, that procedure considered only the overall normalization and did not require the detailed agreement we see comparing Figures 1 and 2.

In the bottom row, we vary our approach to the infrared cirrus. By default, we correct the $24 \mu \mathrm{m}$ map for infrared cirrus following L12. The bottom left panel shows the results of applying no cirrus subtraction, while in the bottom right panel we double our cirrus subtraction. Turning off the cirrus subtraction yields median $\tau_{\text {dep }}^{\text {mol }} \approx 10 \%$ shorter than our best estimate with notably lower scatter and our strongest observed correlation. The tighter correlation reflects the fact that the relationship between $24 \mu \mathrm{m}$ and $\mathrm{CO}$ emission is the strongest in the data (see also Schruba et al. 2011). $\Sigma_{\text {SFR }}$ tracers that more heavily emphasize $24 \mu \mathrm{m}$ exhibit the strongest correlation with $\Sigma_{\text {mol }}$ traced by CO. 
Doubling the cirrus subtraction leads to an $\approx 25 \%$ longer $\tau_{\text {dep }}^{\text {mol }}$, larger scatter, and a mildly weaker correlation between $\Sigma_{\mathrm{SFR}}$ and $\Sigma_{\text {mol }}$. This partially reflects uncertainty in the cirrus calculation, which relies on model fits to observed data. It also reflects the de-emphasis of $24 \mu \mathrm{m}$ emission, which exhibits a very tight correspondence to $\mathrm{CO}$, in favor of $\mathrm{H} \alpha$, which still exhibits a good correspondence but with more scatter. The fraction of data that have upper only limits for $\Sigma_{\mathrm{SFR}}$ also increases, so that extending this analysis to lower surface density will require improved data and methodology.

Thus we observe subtle variations in the relation between $\Sigma_{\text {SFR }}$ and $\Sigma_{\text {mol }}$ depending on the exact treatment of $24 \mu \mathrm{m}$ emission, including up to a $\approx 50 \%$ variation in $\tau_{\text {Dep }}^{\text {mol }}$ across the full plausible range of cirrus treatments. However, our main results of a simple correspondence between $\Sigma_{\text {mol }}$ and $\Sigma_{\text {SFR }}$ hold even when we omit IR data from the analysis. Note that this conclusion relies on the assumption that $\mathrm{H} \alpha$ emission traces recent star formation. If a substantial fraction of $\mathrm{H} \alpha$ emission arises from sources other than recent star formation or if the mean free path of an ionizing photon regularly exceeds one of our kpc-sized resolution elements, then this general agreement may break down (Rahman et al. 2011; Liu et al. 2011; see discussion in L12). These more exotic situations aside, overall Figure 2 and Table 3 show good qualitative and quantitative agreement among different approaches to estimate $\Sigma_{\mathrm{SFR}}$. We will find the same when fitting the data in Section 3.3.

Throughout the rest of the paper, we adopt $\mathrm{H} \alpha+24 \mu \mathrm{m}$, corrected for the effects of a $24 \mu \mathrm{m}$ cirrus, as our single, best estimate of $\Sigma_{\mathrm{SFR}}$. L12 justify this choice, and we refer to that paper for more discussion.

\subsubsection{Choice of CO Line}

HERACLES consists of maps of CO (2-1) emission, which we use to estimate the distribution of $\mathrm{H}_{2}$. $\mathrm{CO}(1-0)$ has been more commonly used to trace the distribution of $\mathrm{H}_{2} \cdot{ }^{12} \mathrm{CO}(1-0)$ maps exist for a subset of our targets (Helfer et al. 2003; Kuno et al. 2007). Though these do not have the same overall quality as the HERACLES maps, we use them to assess the impact of our choice of molecular gas tracer. Figure 3 plots $\Sigma_{\text {SFR }}$ as a function of $\Sigma_{\text {mol }}$ estimated from literature CO (1-0) data. We allow repeats, so that if Helfer et al. (2003) and Kuno et al. (2007) each mapped a galaxy, we include each data set in Figure 3.

Overall, results for $\mathrm{CO}(2-1)$ and $\mathrm{CO}(1-0)$ agree fairly well. The CO (1-0) data tend to yield higher $\tau_{\mathrm{dep}}^{\mathrm{mol}}$. This is exclusively a product of the Kuno et al. data. In the overlap of our sample and the Kuno et al. data, the Kuno et al. data yield median $\tau_{\text {Dep }}^{\text {mol }} \approx 3.3 \mathrm{Gyr}$. Our data yield median $\tau_{\text {Dep }}^{\mathrm{mol}} \approx 2.3 \mathrm{Gyr}$ for the same points. However, for the overlap with BIMA SONG (Helfer et al. 2003), the BIMA SONG CO (1-0) data give median $\tau_{\text {dep }}^{\text {mol }} \approx 2.1$ Gyr. Over the same points, HERACLES implies $\tau_{\text {dep }}^{\text {mol }} \approx 2.2$ Gyr. The disagreement between our CO $(2-1)$ data and CO (1-0) data thus appears no larger than the disagreement among published $\mathrm{CO}(1-0)$ data sets.

\subsubsection{CO-to- $\mathrm{H}_{2}$ Conversion Factor}

In Figures $1-3$ we adopt a fixed CO-to- $\mathrm{H}_{2}$ conversion, $\alpha_{\mathrm{CO}}$. This assumption may be too coarse given the wide range of metallicities, dust-to-gas ratios, and central $\mathrm{CO}$ concentrations in our targets. Figure 4 shows $\Sigma_{\mathrm{SFR}}$ and $\Sigma_{\text {mol }}$ after the application

12 We emphasize that Sandstrom et al. (2012) demonstrate the ability of CO (2-1) to robustly trace molecular gas in our sample (Section 2.2).

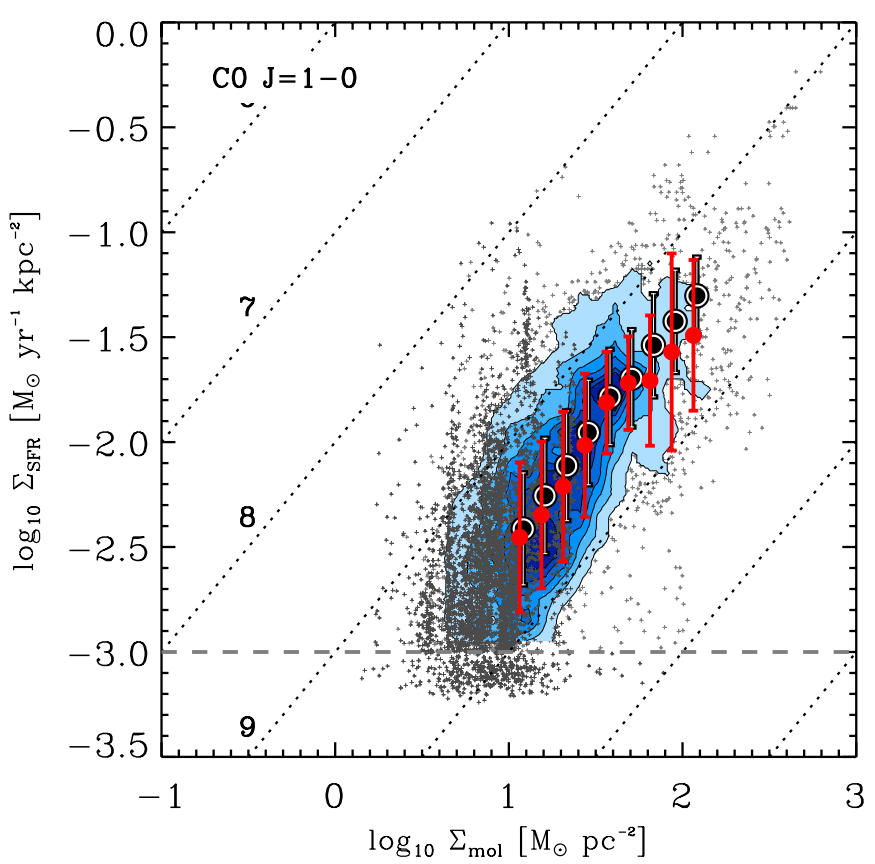

Figure 3. Star formation rate surface density, $\Sigma_{\mathrm{SFR}}$, as a function of molecular gas surface density, $\Sigma_{\text {mol }}$, here estimated from literature CO (1-0) data (Section 2). Gray points show individual measurements, contours indicate data density, and red points show the running median and standard deviation using each tracer. For comparison, large black-and-white circles show the same bins from Figure 1.

of our " $\Sigma=100 "$ conversion factor (Section 2.2), which attempts to account for the presence of $\mathrm{CO}$-poor envelopes of molecular clouds and central CO depressions. This conversion factor assumes that all $\mathrm{CO}$ emission originates from clouds with surface densities of $100 M_{\odot} \mathrm{pc}^{-2}$ with photodissociation region structure like that described in Wolfire et al. (2010). The resulting dependence of $\alpha_{\mathrm{CO}}$ on metallicity approximates the current consensus (Wolfire et al. 2010; Glover \& Mac Low 2011; Leroy et al. 2011; Feldmann et al. 2012; Narayanan et al. 2012), but note that this remains highly uncertain because of limited observational constraints. We also report results for the " $\Sigma=50$ " conversion factor in Table 3 . This conversion factor makes the more aggressive assumption that a large amount of molecular emission emerges from weakly shielded parts of clouds, rendering $\alpha_{\mathrm{CO}}$ very sensitive to the dust-to-gas ratio.

Applying the " $\Sigma=100 " \alpha_{\mathrm{CO}}$ to the data in Figure 1 yields Figure 4. The top rows, which show the bulk distribution of the data, appear qualitatively similar in the two plots, though Table 3 and close inspection of the plots do indicate that the normalization of the $\Sigma_{\mathrm{SFR}}-\Sigma_{\mathrm{mol}}$ relation changes between the two plots. The median $\tau_{\text {dep }}^{\text {mol }}$ weighting each line of sight equally rises from $2.2 \mathrm{Gyr}$ to $2.6 \mathrm{Gyr}$ with the application of the variable conversion factor.

The most dramatic contrast between Figures 1 and 4 appears in the bottom rows. Many of the low $\tau_{\text {dep }}^{\text {mol }}$ (high $\Sigma_{\mathrm{SFR}}$ ) data in Figure 1 arise from small galaxies with low dust-to-gas ratios. With the application of a variable conversion factor, our estimate of $\Sigma_{\text {mol }}$ in these galaxies moves to higher values while $\Sigma_{\text {SFR }}$ remains constant. The result, visible in the bottom rows of Figure 4, is that data from these low-mass, lowmetallicity galaxies now overlap the other points, forming a (more) continuous single $\Sigma_{\mathrm{SFR}}-\Sigma_{\text {mol }}$ trend. Table 3 reports that the scatter among galaxies drops from $\approx 0.3 \mathrm{dex}$ to $\approx 0.2 \mathrm{dex}$ 


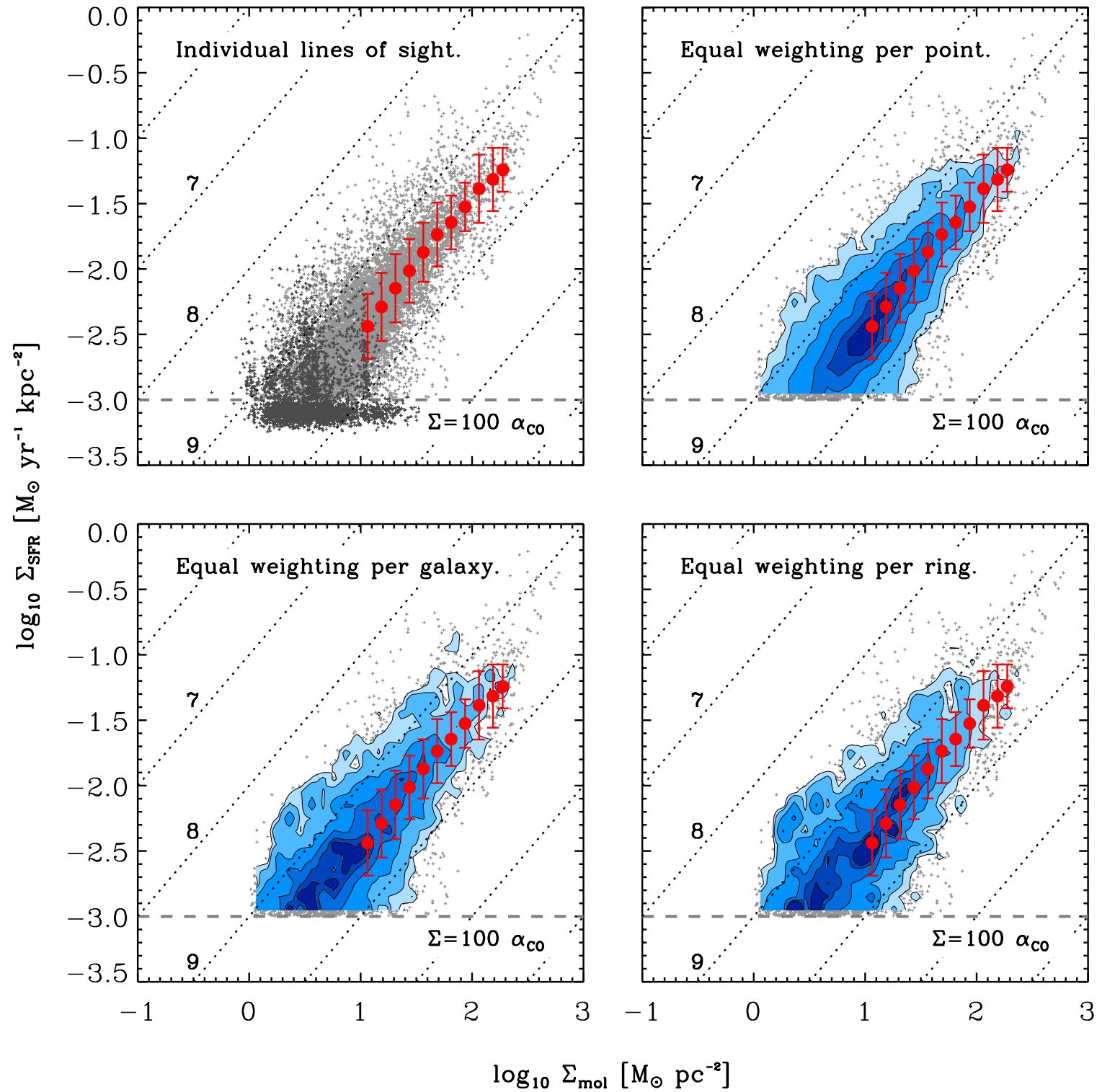

Figure 4. Star formation rate surface density, $\Sigma_{\mathrm{SFR}}$, as a function of molecular gas surface density, $\Sigma_{\text {mol }}$, here estimated from CO (2-1) data combined with our " $\Sigma=100$ " conversion factor $\alpha_{\mathrm{CO}}$, which reflects variations in the dust-to-gas ratio and the central depressions in $\alpha_{\mathrm{CO}}$ found by Sandstrom et al. (2012). Panels and annotations are as in Figure 1.

with the application of the " $\Sigma=100$ " conversion factor, with the systematic difference in $\tau_{\text {dep }}^{\text {mol }}$ between high- and low-mass galaxies reduced from a factor of $\sim 4$ to a factor of $\sim 2$.

Thus, as we will see in Section 4, application of a dustto-gas ratio-dependent conversion factor $\mathrm{CO}-$ to- $\mathrm{H}_{2}$ conversion factor does affect the derived $\Sigma_{\mathrm{SFR}}-\Sigma_{\mathrm{mol}}$ relation, with the sense of moving many points with low apparent $\tau_{\text {dep }}^{\text {mol }}$ into closer agreement with the distribution defined by large galaxies. This scenario of a rapidly varying conversion factor and a

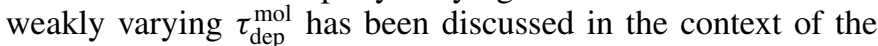
Small Magellanic Cloud by Bolatto et al. (2011) and in a theoretical context by Krumholz et al. (2011). They interpret weak variations of $\tau_{\mathrm{dep}}^{\mathrm{mol}}$ but strong variations of $\alpha_{\mathrm{CO}}$ as evidence that the requisite preconditions for star formation more closely resemble those for $\mathrm{H}_{2}$ formation than those required for a high $\mathrm{CO}$ abundance.

The other portion of the " $\Sigma=100$ " conversion factor, the $\alpha_{\mathrm{CO}}$ depressions in the central parts of galaxies, affects too few data to be prominent in Figure 4. However, one can see many individual low $\tau_{\text {dep }}^{\text {mol }}$ points at moderate $\Sigma_{\text {mol }}$ on close inspection. We return to this point in Section 4.4.

\subsection{Power-law Index}

Studies of the star formation-gas connection in galaxies have treated the relationship as a power-law and focused on the index of this power-law. While this single parameter undoubtedly makes for easy shorthand, the fixation on this parameter obscures environmental factors other than $\Sigma_{\text {gas }}$. Recent observations offer good evidence that $\Sigma_{\mathrm{SFR}}$ relates to $\Sigma_{\mathrm{HI}}$ and $\Sigma_{\mathrm{mol}}$ in fundamentally different ways (L08; B08; Wong \& Blitz 2002; Schruba et al. 2011) and that $\Sigma_{\mathrm{SFR}}$ is even a multivalued function of $\Sigma_{\text {mol }}$ (Daddi et al. 2010; Genzel et al. 2010; Schruba et al. 2011; Saintonge et al. 2011, 2012; and this paper).

Despite the shortcomings of this approach, we consider the best-fit index in our data as a useful, or at least expected, point of 
comparison to previous studies. We derive best-fit relations for our ensemble of measurements and individual galaxies. We fit a relation with three parameters: a normalization, $a$, power-law index, $n$, and intrinsic, log-normally distributed scatter with rms magnitude $s$. Then

$$
\Sigma_{\mathrm{SFR}}=a\left(\frac{\Sigma_{\mathrm{gas}}}{10 M_{\odot} \mathrm{pc}^{2}}\right)^{n}
$$

with data intrinsically scattered by $s$. We derive the best-fit $a$, $n$, and $s$ using a Monte Carlo approach based on the work of Blanc et al. (2009). This resembles Hess diagram fitting used for optical color-magnitude diagrams. It includes observational uncertainties, upper limits, and intrinsic scatter in the relation. This approach also avoids important biases that can easily arise fitting scaling relations to noisy, bivariate data. We illustrate these biases, which affect many commonly adopted approaches, in Appendix C (see also Blanc et al. 2009) and note that they can easily shift the derived index by a few tenths for realistic data distributions.

Following Blanc et al. (2009) we grid our data, deriving a twodimensional image of data density in regularly spaced cells in $\log _{10} \Sigma_{\mathrm{SFR}}-\log _{10} \Sigma_{\text {mol }}$ space. Unlike Blanc et al. (2009) we work in logarithmic space. This gives us a better ability to resolve the distribution of our data, but forces a coarser approach to upper limits. We treat upper limits by essentially creating an "upper limit row" along the $\Sigma_{\text {SFR }}$ axis. In detail, we adopt the following approach.

1. We exclude all data with $\Sigma_{\text {mol }}<5 M_{\odot} \mathrm{pc}^{-2}$. This gives us a data set with a well-defined $x$-axis.

2. We generate Monte Carlo data sets for a wide range of $a$, $n$, and $s$ in the following way. We take our observed $\Sigma_{\text {mol }}$ to represent the true physical distribution. We draw 100,000 data points from this distribution (allowing repeats) for each combination of $a, n$, and $s$. We derive $\Sigma_{\mathrm{SFR}}$ for each of these points. We then apply the expected uncertainty to $\Sigma_{\text {mol }}$ (the statistical uncertainty from HERACLES) and $\Sigma_{\text {SFR }}(0.15$ dex $)$. We grid these data in $\log _{10} \Sigma_{\text {SFR }}-\log _{10} \Sigma_{\text {mol }}$ space, using cells 0.125 dex wide in both dimensions. We treat this grid as the expected probability distribution function for those underlying parameters $a, n$, and $s$.

3. We grid our observed data in $\log _{10} \Sigma_{\mathrm{SFR}}-\log _{10} \Sigma_{\text {mol }}$ using the same grid on which we derived probability distribution functions. We create different grids for each set of $\Sigma_{\text {SFR }}$ estimates.

4. We compare our gridded data to the Monte Carlo realization for each $a, n, s$ combination and calculate a goodness-of-fit estimate, which we here loosely refer to as $\chi^{2}$. After renormalizing the Monte Carlo grid to have the same amount of data as the observed grid, we calculate

$$
\chi^{2}=\sum_{i} \frac{\left(N_{\mathrm{obs}}^{i}-N_{\text {model }}^{i}\right)^{2}}{N_{\text {model }}^{i}},
$$

where the sum runs across all cells, $i, N_{\text {obs }}$ refers to the observed number of data in the grid cell, and $N_{\text {model }}$ refers to the expected number of data in that cell given $a, n$, and $s$ and our observational uncertainties. The goodness-of-fit statistic is thus analogous to $\chi^{2}$ calculated for the case of Poisson noise in each cell. Points with only upper limits on $\Sigma_{\mathrm{SFR}}\left(\right.$ where $\Sigma_{\mathrm{SFR}}<10^{-3} M_{\odot} \mathrm{yr}^{-1} \mathrm{kpc}^{-2}$ ) are included in
Table 4

Results of Monte Carlo Fitting to Equation (9)

\begin{tabular}{lcrr}
\hline \hline Tracer & $\begin{array}{c}\log _{10} a \\
\left(M_{\odot} \mathrm{yr}^{-1} \mathrm{kpc}^{-2}\right)\end{array}$ & $n$ & \multicolumn{1}{c}{\begin{tabular}{c}
\multicolumn{1}{c}{$\begin{array}{c}\text { (dex }) \\
\end{array}$} \\
\hline $\mathrm{H} \alpha+24 \mu \mathrm{m}$
\end{tabular}} \\
$\ldots$ no cirrus removed & $-2.40 \pm 0.09$ & $0.95 \pm 0.15$ & $0.30 \pm 0.05$ \\
$\ldots$ double cirrus removed & $-2.55 \pm 0.08$ & $0.95 \pm 0.13$ & $0.225 \pm 0.05$ \\
FUV $+24 \mu \mathrm{m}$ & $-2.40 \pm 0.09$ & $0.95 \pm 0.16$ & $0.30 \pm 0.05$ \\
$\mathrm{H} \alpha$ with $A_{\mathrm{H} \alpha}=1 \mathrm{mag}$ & $-2.35 \pm 0.09$ & $0.80 \pm 0.14$ & $0.275 \pm 0.06$ \\
\hline$\Sigma=100 \alpha_{\mathrm{CO}}$ and $\mathrm{H} \alpha+24 \mu \mathrm{m}$ & $-2.45 \pm 0.09$ & $1.0 \pm 0.15$ & $0.30 \pm 0.05$ \\
$\Sigma=50 \alpha_{\mathrm{CO}}$ and $\mathrm{H} \alpha+24 \mu \mathrm{m}$ & $-2.35 \pm 0.09$ & $0.90 \pm 0.15$ & $0.30 \pm 0.05$ \\
\hline
\end{tabular}

Notes. Results of Monte Carlo fitting to Equation (9) for different combinations of $\Sigma_{\mathrm{SFR}}$ and $\Sigma_{\mathrm{mol}}$ tracers. Column 1 reports the tracer used; in the top five rows we vary the $\Sigma_{\mathrm{SFR}}$ tracer while adopting fixed $\alpha_{\mathrm{CO}}$. The last two rows adopt our best $\Sigma_{\mathrm{SFR}}$ estimate, $\mathrm{H} \alpha+24 \mu \mathrm{m}$, and vary the adopted conversion factor. Columns 2-4 report the best-fit coefficient at $\Sigma_{\text {mol }}=10 M_{\odot} \mathrm{pc}^{-2}$, the powerlaw index, and the intrinsic scatter. We quote uncertainties from the Monte Carlo simulations described in Appendix C.

the calculation. These have an associated $\Sigma_{\text {mol }}$ value, but all upper limits are treated as having the same $\Sigma_{\mathrm{SFR}}$.

We apply this method to our ensemble of data, repeating the exercise for each SFR tracer discussed in Section 3.2 and for our fixed, " $\Sigma=100$," and " $\Sigma=50$ " conversion factors. We also fit each galaxy on its own. ${ }^{13}$ Figure 5 and Table 4 report our fits to the combined data set. Figure 5 plots the approximate reduced $\chi^{2}$ as a function of power-law index, marginalizing over $a$ and $s$. We observe clear minima in the range $n=0.5-1.5$ for all SFR tracers. Appendix C presents a Monte Carlo treatment that considers a number of effects: robustness to removal of individual data or galaxies, statistical noise, calibration (gain) uncertainties for each data set, and exact choice of fitting methodology. We quote uncertainties derived from this Monte Carlo treatment in Table 4.

The fits in Table 4 suggest a power-law with $n \approx 1 \pm 0.15$, intrinsic scatter of a factor of $\approx 2(0.3 \mathrm{dex})$, and $\tau_{\mathrm{dep}}^{\mathrm{mol}} \approx 2.5$ Gyr at $\Sigma_{\mathrm{mol}}=10 M_{\odot} \mathrm{pc}^{-2}$. The slope remains consistent with a linear relation between $\mathrm{H}_{2}$ and star formation (B08; Bigiel et al. 2011) or with the weakly super-linear slope of Genzel et al. (2010) or Daddi et al. (2010), though note that our $1 \mathrm{kpc}$ scale does not precisely match their observations. The mild difference between the best-fit coefficient, $a$, and the median $\tau_{\text {dep }}^{\text {mol }}$ reported in Table 3 reflects the inadequacy of the power-law to capture the full distribution of the data.

The choice of SFR tracer affects the fit, but offers more of a refinement than a qualitative shift in these conclusions. The sense of the shifts resembles those seen in Section 3.2. Replacing FUV for $\mathrm{H} \alpha$ as the unobscured tracer has minimal effect. Using only $\mathrm{H} \alpha$ to estimate SFR yields a slightly shallower slope. Based on the observed $\mathrm{H} \alpha$-to-IR ratio, extinction increases with increasing $\Sigma_{\mathrm{SFR}}$ (see plots in Prescott et al. 2007 and L12). By assuming a fixed $A_{\mathrm{H} \alpha}$ we would expect to underestimate $\Sigma_{\mathrm{SFR}}$ at the high end and overestimate it at the low end, somewhat "tilting" the relationship to shallower slope. If we do not remove any cirrus from the data, working only with the measured $24 \mu \mathrm{m}$ emission, the scatter in the relation diminishes to less than 0.2 dex. This underscores the point that it is the tight observed correlation between $\mathrm{CO}$ and $24 \mu \mathrm{m}$ emission that drives much

\footnotetext{
13 Due to the lower density of points, we use 0.2 dex cell sizes and require
} only 10,000 points to populate the theoretical distribution. 

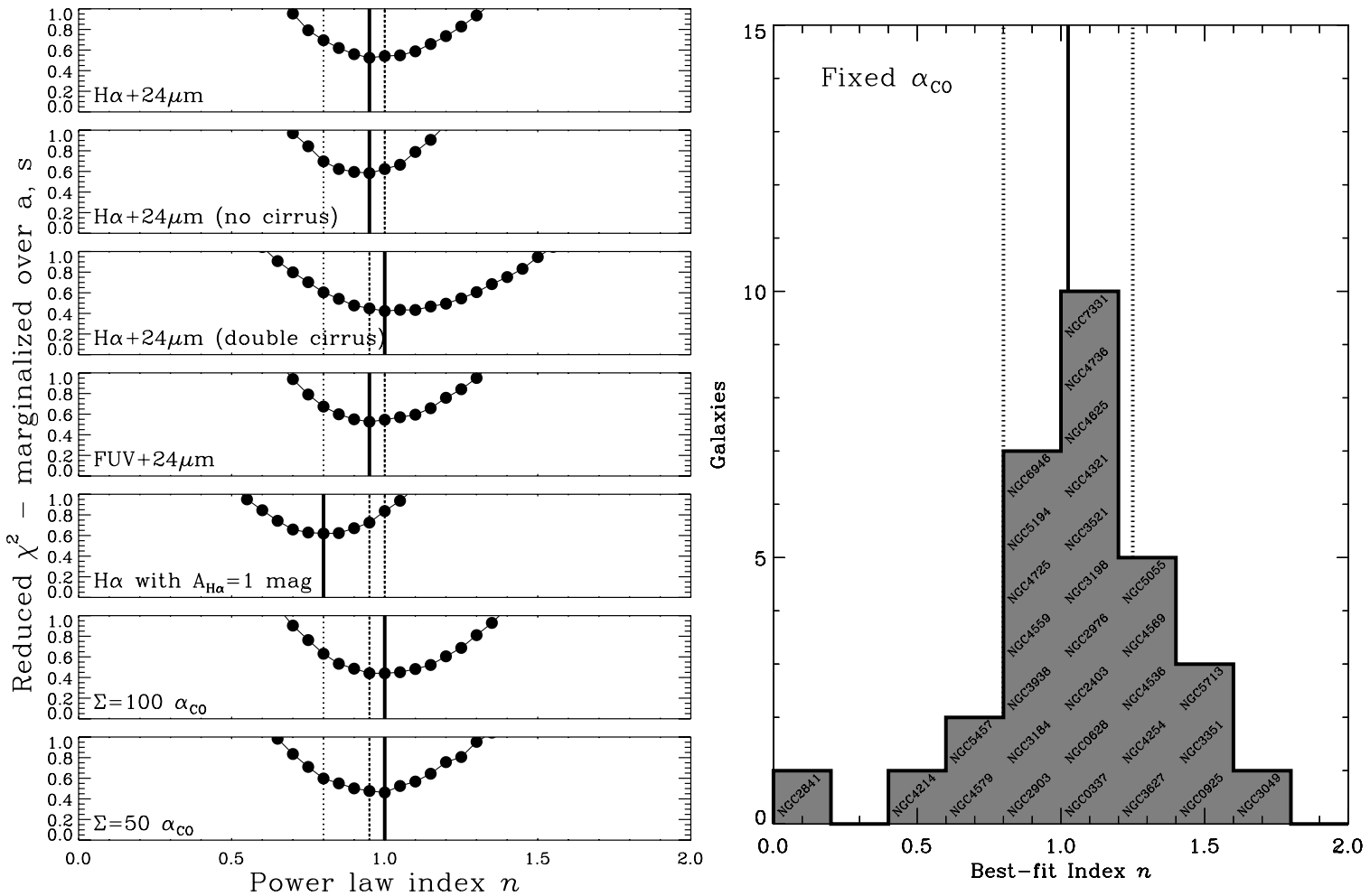

Figure 5. Results of power-law fitting for (left) our combined data set and (right) individual galaxies. Left: goodness of fit as a function of power-law index, $n$, for fits to our entire ensemble of data where $\Sigma_{\mathrm{mol}}>5 \mathrm{M}_{\odot} \mathrm{pc}^{-2}$. The fitting uses a modified version of the Monte Carlo technique outlined by Blanc et al. (2009). From top to bottom, we show results for fixed $\alpha_{\mathrm{CO}}$ using our best $\mathrm{H} \alpha+24 \mu \mathrm{m}$ and FUV $+24 \mu \mathrm{m}$ estimates; $\mathrm{H} \alpha+24 \mu \mathrm{m}$ with no cirrus removed or double the best-estimate cirrus removed; and $\mathrm{H} \alpha$ with an assumed fixed 1 magnitude of extinction. We also show results for our " $\Sigma=100 "$ and " $\Sigma=50$ " conversion factors. Vertical lines indicate where $n$ reaches its minimum value, bold for the value in that panel, dotted for other panels. Each case shows a broad minimum in $\chi^{2}$ with a best-fit value near $n \approx 1 \pm 0.2$. Right: best-fit power-law index, $n$, for individual galaxies using $\mathrm{H} \alpha+24 \mu \mathrm{m}$ and a fixed CO-to- $\mathrm{H}_{2}$ conversion factor. The distribution of best-fit $n$ for individual galaxies peaks near $n \approx 1$ with most galaxies in the range $n \approx 0.8-1.2$ but outliers up to $n \gtrsim 1.5$.

of the recent work on this topic (see more discussion in Rahman et al. 2011; Schruba et al. 2011; Liu et al. 2011) so that SFR tracers that emphasize $24 \mu \mathrm{m}$ data tend to yield the tightest relations. Conversely, increasing the cirrus removed leads to a somewhat longer overall $\tau_{\mathrm{dep}}^{\mathrm{mol}}$ with larger intrinsic scatter. Adjusting the conversion factor exerts only a mild impact on the fit because largest corrections apply to small galaxies and often to low apparent $\Sigma_{\text {mol }}$ regions. These significant variations to a small subset of the data do not drive substantial variations in the fit.

The simple nearly linear scaling given by our fits could result from the superposition of a varied set of distinct relations for individual galaxies (see Schruba et al. 2011). In the right-hand panel of Figure 5 we show that indeed the best-fit index for individual galaxies exhibits significant scatter. We find a median 1.05 , but best-fit values span $\sim 0.5-1.5$. We report best-fit indices for individual galaxies in the Appendix and stress two general conclusions here. First, we see variation in index from galaxy to galaxy, but the $67 \%$ range is still $0.8-1.25$, consistent with the idea that to first order the molecular gas supply regulates the star formation distribution and in sharp contrast to the steep indices relating $\Sigma_{\text {SFR }}$ to atomic gas (B08; Schruba et al. 2011). Second, the fact that these galaxy-to-galaxy variations wash out into Figure 1 implies that while $\tau_{\text {dep }}^{\text {mol }}$ may correlate with $\Sigma_{\text {mol }}$ within an individual galaxy, simply knowing $\Sigma_{\text {mol }}$ at $1 \mathrm{kpc}$ resolution with no other knowledge of local conditions or host galaxy does not allow one to predict $\tau_{\text {dep }}^{\text {mol better than }}$ simply adopting a median $\tau_{\text {dep }}^{\text {mol }}$. That is, in the absence of knowledge of other conditions, $\Sigma_{\text {mol }}$ at our resolution and in our sample is not a good predictor of the molecular gas depletion time.

\subsection{Comparison to Literature Data}

Many studies have assessed the relationship between gas and star formation in nearby galaxies (Section 1). Figure 6 and Table 5 compare our measurements to a compilation of these studies (see Section 2.5 and Bigiel et al. 2011). We adjust each set of measurements to share our adopted $\mathrm{CO}-$ to- $\mathrm{H}_{2}$ conversion factor and stellar IMF.

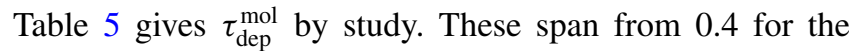
Kennicutt (1998b) starbursts to $4.4 \mathrm{Gyr}$ for the study of NGC 6946 by Crosthwaite \& Turner (2007). Considering all measurements equally, the median literature $\tau_{\mathrm{dep}}^{\mathrm{mol}}$ is $2.7 \mathrm{Gyr}$, which drops to $2.1 \mathrm{Gyr}$ if we exclude the large data set of Rahman et al. (2012), which otherwise dominates the statistics. Treating each study as a single independent measurement, the median is $\tau_{\text {dep }}^{\mathrm{mol}}=2.0 \mathrm{Gyr}$. These are in good agreement with the estimates of this study (Table 3) $\tau_{\text {dep }}^{\text {mol }} \approx 2.2$ Gyr weighting all measurements equally. The scatter among individual literature data is $\approx 0.36 \mathrm{dex}$, and from study to study the scatter is $\approx 0.23$ dex.

Figure 6 shows a more detailed comparison between our measurements and individual literature data. We separate the literature studies according to the scale sampled. The top left panel shows measurements where one point corresponds to one galaxy. The top right panel shows data from studies that measure azimuthally averaged $\Sigma_{\mathrm{mol}}$ and $\Sigma_{\mathrm{SFR}}$ in a series of concentric tilted rings. The bottom left panel shows data for 

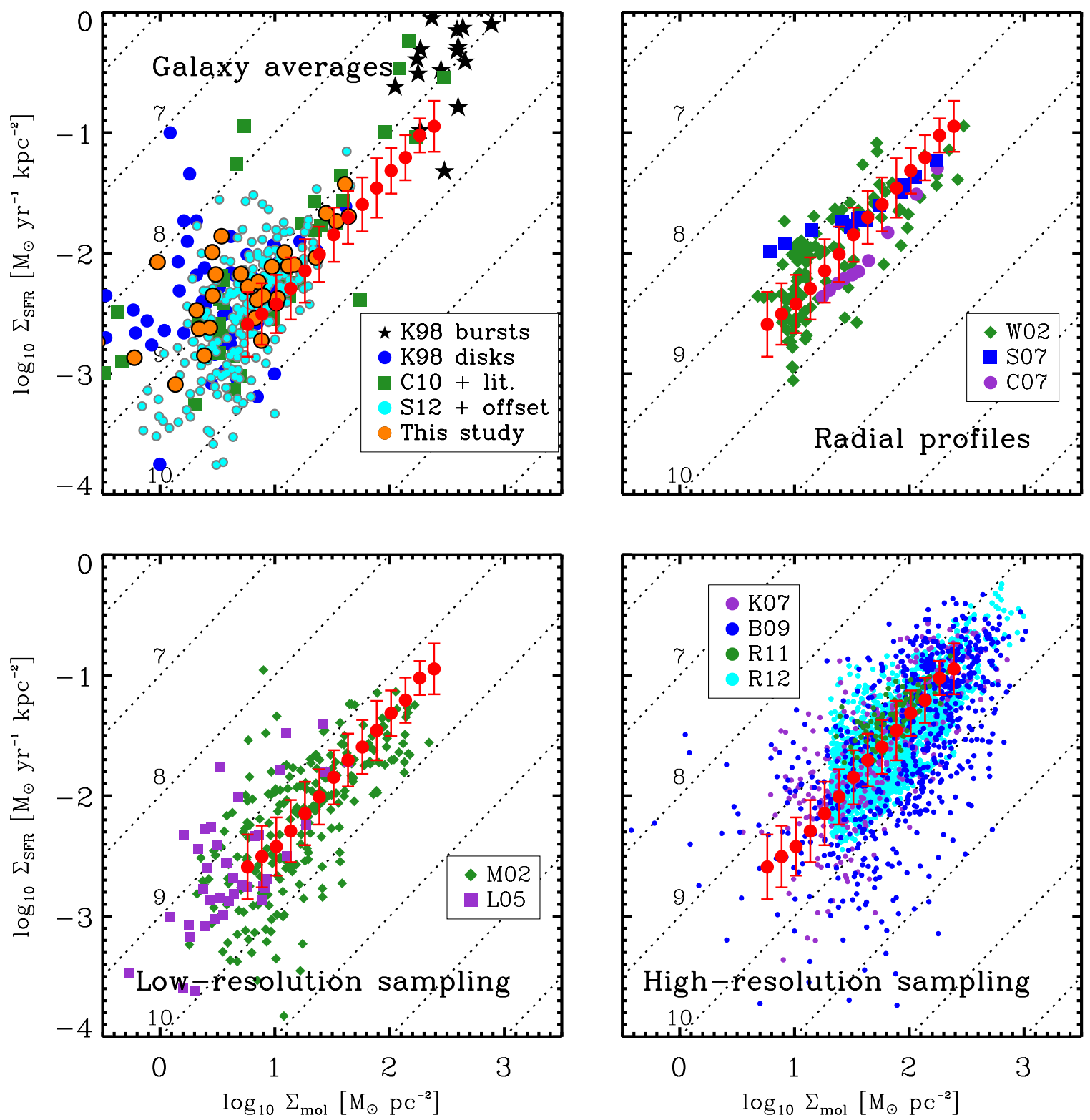

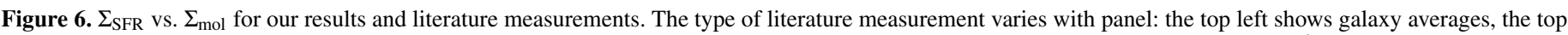

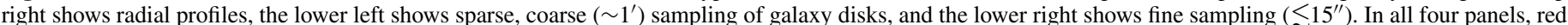

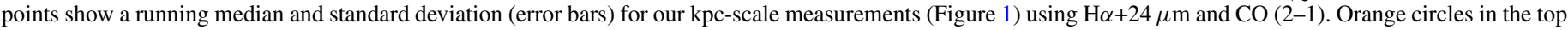

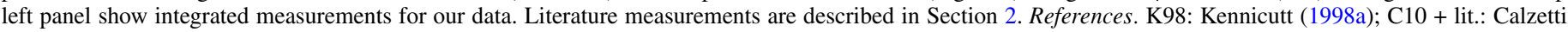

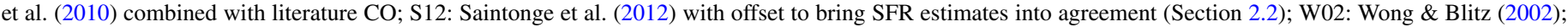

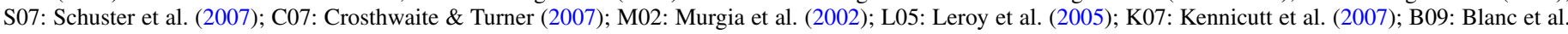
(2009); R11: Rahman et al. (2011); R12: Rahman et al. (2012).

individual pointings with comparatively poor angular resolution, $\approx 40^{\prime \prime}-60^{\prime \prime}$. The bottom right panel shows studies that obtain high-angular-resolution sampling of each target. In each panel we plot the running median and standard deviation for our data, binned by $\Sigma_{\text {mol }}$, as red points.

The final three panels of Figure 6 demonstrate excellent agreement between our data and previous studies that resolve the disks of galaxies (see also Bigiel et al. 2011). This agreement may not be surprising given that our study shares targets with many of these literature studies, which also heavily overlap one another. Nonetheless, we show here that repeated measurements of the distribution of $\Sigma_{\mathrm{SFR}}$ and $\Sigma_{\mathrm{mol}}$ in the nearest star-forming spiral galaxies mostly cover the same part of parameter space regardless of exact methodology. Uncertainty in interpretation and fitting techniques have clouded this basic agreement in where the data lie. Given our basic approach to physical parameter estimation, there appears to be overall agreement for a typical $\tau_{\mathrm{dep}}^{\mathrm{mol}}=1-3 \mathrm{Gyr}$ in local disk galaxies.

The first panel of Figure 6 looks qualitatively different from the other three. This panel shows galaxy-integrated measurements, so that one point is one galaxy. These tend to scatter from overlapping our data up to lower $\tau_{\text {dep }}^{\text {mol }}$ at low $\Sigma_{\text {mol }}$ and comparatively high $\Sigma_{\text {SFR }}$. This same effect appears in Table 5 as low values of $\tau_{\mathrm{dep}}^{\mathrm{mol}}$ for studies that focus on measurements of whole galaxies. Our synthesis of literature CO and SFR measurements yields $\tau_{\mathrm{dep}}^{\mathrm{mol}}=1.3 \mathrm{Gyr}$, while for the Kennicutt (1998a) disk galaxies the median $\tau_{\text {dep }}^{\text {mol }}=1.1$ Gyr. Treating our own sample as a set of integrated measurements we find a similar value, $\tau_{\mathrm{dep}}^{\mathrm{mol}}=1.3 \mathrm{Gyr}$. 
Table 5

Comparison to Literature

\begin{tabular}{|c|c|c|}
\hline Galaxy & $\begin{array}{c}\text { Median } \tau_{\mathrm{dep}}^{\mathrm{mol}} \\
(\mathrm{Gyr})\end{array}$ & $\begin{array}{l}\text { Scatter } \\
\text { (dex) }\end{array}$ \\
\hline \multicolumn{3}{|l|}{ This study ... } \\
\hline ... weighting by measurement & 2.2 & 0.28 \\
\hline ... weighting by galaxy & 1.3 & 0.32 \\
\hline \multicolumn{3}{|l|}{ Median of literature ... } \\
\hline ... weighting by measurement & $2.7^{\mathrm{a}}$ & 0.36 \\
\hline ... weighting by study & 2.0 & 0.23 \\
\hline \multicolumn{3}{|c|}{ Disk Averages } \\
\hline Saintonge et al. (2012) & 0.7 & 0.37 \\
\hline$\ldots$ offset SFR $^{b}$ & 1.1 & 0.37 \\
\hline Calzetti et al. $(2010)+$ literature $\mathrm{CO}^{\mathrm{c}}$ & 1.5 & 0.38 \\
\hline \multicolumn{3}{|l|}{ Kennicutt $(1998 b) \ldots$} \\
\hline$\ldots$ disks & 1.1 & 0.46 \\
\hline$\ldots$ starbursts & 0.4 & 0.39 \\
\hline \multicolumn{3}{|c|}{ Radial Profiles } \\
\hline Schuster et al. (2007) & 2.0 & $0.12^{\mathrm{d}}$ \\
\hline Crosthwaite \& Turner (2007) & 4.4 & $0.06^{\mathrm{d}}$ \\
\hline Wong \& Blitz (2002) & 2.0 & 0.36 \\
\hline \multicolumn{3}{|c|}{ Low Resolution } \\
\hline Leroy et al. (2005) & 2.1 & 0.33 \\
\hline Murgia et al. (2002) & 2.8 & 0.41 \\
\hline \multicolumn{3}{|c|}{ High Resolution } \\
\hline Rahman et al. (2012) & 2.9 & 0.37 \\
\hline Rahman et al. (2011) & 1.6 & $0.15^{\mathrm{d}}$ \\
\hline Blanc et al. (2009) & 3.2 & $0.64^{\mathrm{d}}$ \\
\hline Kennicutt et al. (2007) & 2.2 & $0.37^{\mathrm{d}}$ \\
\hline
\end{tabular}

Notes. Average molecular gas depletion time, in Gyr, for matched assumptions $-X_{\mathrm{CO}}=2 \times 10^{20} \mathrm{~cm}^{-2}\left(\mathrm{~K} \mathrm{~km} \mathrm{~s}^{-1}\right)^{-1}$, a Kroupa IMF, and including helium in the gas estimate. The left column gives the study, with the list broken down by sampling approach, and the right column reports the median molecular gas depletion time in that study. Error bars report the $1 \sigma$ scatter, in dex, for each study.

a Dominated by Rahman et al. (2012). Without Rahman et al. (2012) median is 2.1 Gyr.

b SFR estimate offset to match our estimates (Section 2.2).

${ }^{c}$ CO from Young et al. (1995) and Helfer et al. (2003).

d Study considered a single galaxy. Others combine multiple galaxies.

This disk-integrated $\tau_{\mathrm{dep}}^{\mathrm{mol}}$ is significantly shorter than the $\tau_{\mathrm{dep}}^{\mathrm{mol}}$ that we measure treating each point equally. We noted this effect in Section 3.1. It arises because weighting each galaxy equally emphasizes small, low-mass, low-SFR galaxies relative to large, massive galaxies. These low-mass galaxies have less physical area than large disks, so that they do not affect the ensemble of measurements much. However, these small galaxies do exhibit short apparent $\tau_{\text {dep }}^{\text {mol }}$ and when given equal weight they drive the

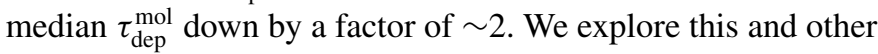
systematic variations in $\tau_{\mathrm{dep}}^{\mathrm{mol}}$ in the second part of this paper.

\section{SYSTEMATIC SECOND-ORDER VARIATIONS IN $\tau_{\text {dep }}^{\text {mol }}$ : GLOBAL CORRELATIONS, EFFICIENT GALAXY CENTERS, AND CORRELATED SCATTER}

In Section 3 we demonstrate that our ensemble of data can be described to first order by a roughly linear relation between $\Sigma_{\mathrm{SFR}}$ and $\Sigma_{\text {mol }}$ with a slope corresponding to a typical depletion time $\tau_{\mathrm{dep}}^{\mathrm{mol}} \approx 2.2 \mathrm{Gyr}$ with a factor of two scatter from line of sight to line of sight. However, we also show that the apparent uniformity of $\tau_{\mathrm{dep}}^{\mathrm{mol}}$ results at least partially from the emphasis that our approach places on the disks of large, star-forming galaxies. These contribute most of the area in our sample. When we apply weightings that emphasize small galaxies or the inner parts of galaxies, we observe departures from this simple picture.

In this section, we explore these variations. We examine the dependence of disk-average $\tau_{\mathrm{dep}}^{\mathrm{mol}}$ on integrated galaxy properties (Section 4.1) and the dependence of $\tau_{\mathrm{dep}}^{\mathrm{mol}}$ on local physical conditions (Section 4.2). We highlight apparent variations in $\tau_{\text {dep }}^{\text {mol }}$ as a function of galaxy mass, metallicity, and dust-togas ratio and we discuss the $\mathrm{CO}$-to- $\mathrm{H}_{2}$ conversion factor as a potential cause. In Section 4.4 we contrast the central regions of our targets with the disks and show evidence for a shift to more efficient star formation in galaxy centers, perhaps indicative of a transition between "disk" and "starburst" modes of star formation. Finally we examine the scale-dependence of scatter in $\tau_{\text {dep }}^{\text {mol }}$ (Section 4.3) to show that undiagnosed systematic variations in $\tau_{\text {dep }}^{\text {mol }}$ persist in our data, reflecting either large-scale synchronization of star formation or real correlated efficiency variations spanning the disks of our targets.

\subsection{Galaxy-to-galaxy Variations in $\tau_{d e p}^{m o l}$}

Our comparison with the literature reveals the same effect that we saw in Section 3.1: when we weight by host galaxy instead of by individual kpc-scale measurement, clear variations in $\tau_{\text {dep }}^{\text {mol }}$ become visible. In particular, a large population of short $\tau_{\text {dep }}^{\text {mol }}$ data emerges in the bottom left panel of Figure 1 and the top left panel of Figure 6. In Figure 7 we investigate the physical origin of these differences. Adopting a fixed $\alpha_{\mathrm{CO}}$, we plot $\tau_{\mathrm{dep}}^{\mathrm{mol}}$ for each galaxy in our sample as a function of a series of galaxy properties: stellar mass, disk-average total ( $\mathrm{H} \mathrm{I}+$ $\mathrm{H}_{2}$ ) gas surface density, metallicity, dust-to-gas ratio, rotation velocity, average orbital time (weighted by $\Sigma_{\text {mol }}$ ), morphological type, and specific star formation rate $\left(\mathrm{sSFR}=\mathrm{SFR} / M_{*}\right) \cdot \tau_{\mathrm{dep}}^{\mathrm{mol}}$ correlates with many of these quantities with the overall sense that low-mass, low-metallicity, late-type, high-sSFR galaxies exhibit shorter apparent molecular gas depletion times than massive, metal-rich, earlier type disk galaxies.

Such trends have been observed in various ways before. In Figure 7, gray points show the approximate $\tau_{\text {dep }}^{\text {mol }}$ as a function of morphology as inferred by Young et al. (1996) from comparing the FCRAO Extragalactic CO Survey with $\mathrm{H} \alpha$ (squares) and IR (diamonds) data. They found later-type galaxies to exhibit mildly enhanced star formation efficiencies. We also plot $\tau_{\mathrm{dep}}^{\mathrm{mol}}$ derived by Krumholz et al. (2011) combining literature SFR and CO data (including HERACLES measurements), who showed a strong trend of SFR/CO decreasing with increasing metallicity (see also Schruba et al. 2012). Both literature trends agree well with our own data, and here we present the first direct comparison to the dust-to-gas ratio, the quantity thought to link metallicity to both $\mathrm{CO}$ emission and the star formation process (e.g., Wolfire et al. 2010; Krumholz et al. 2011).

Saintonge et al. $(2011,2012)$ found a clear relation between $\tau_{\text {dep }}^{\text {mol }}$ and $M_{*}$ and identified sSFR as the global galaxy property most directly linked to $\tau_{\text {dep }}^{\text {mol }}$. We plot their data, corrected to bring our SFR estimates into agreement in the top row and bottom right panel. In the bottom right panel, we plot their fit of $\tau_{\mathrm{dep}}^{\mathrm{mol}}$, corrected for different treatments of helium. We also indicate the rotation velocity threshold below which Dalcanton et al. (2004) noted the disappearance of prominent dust lanes in 

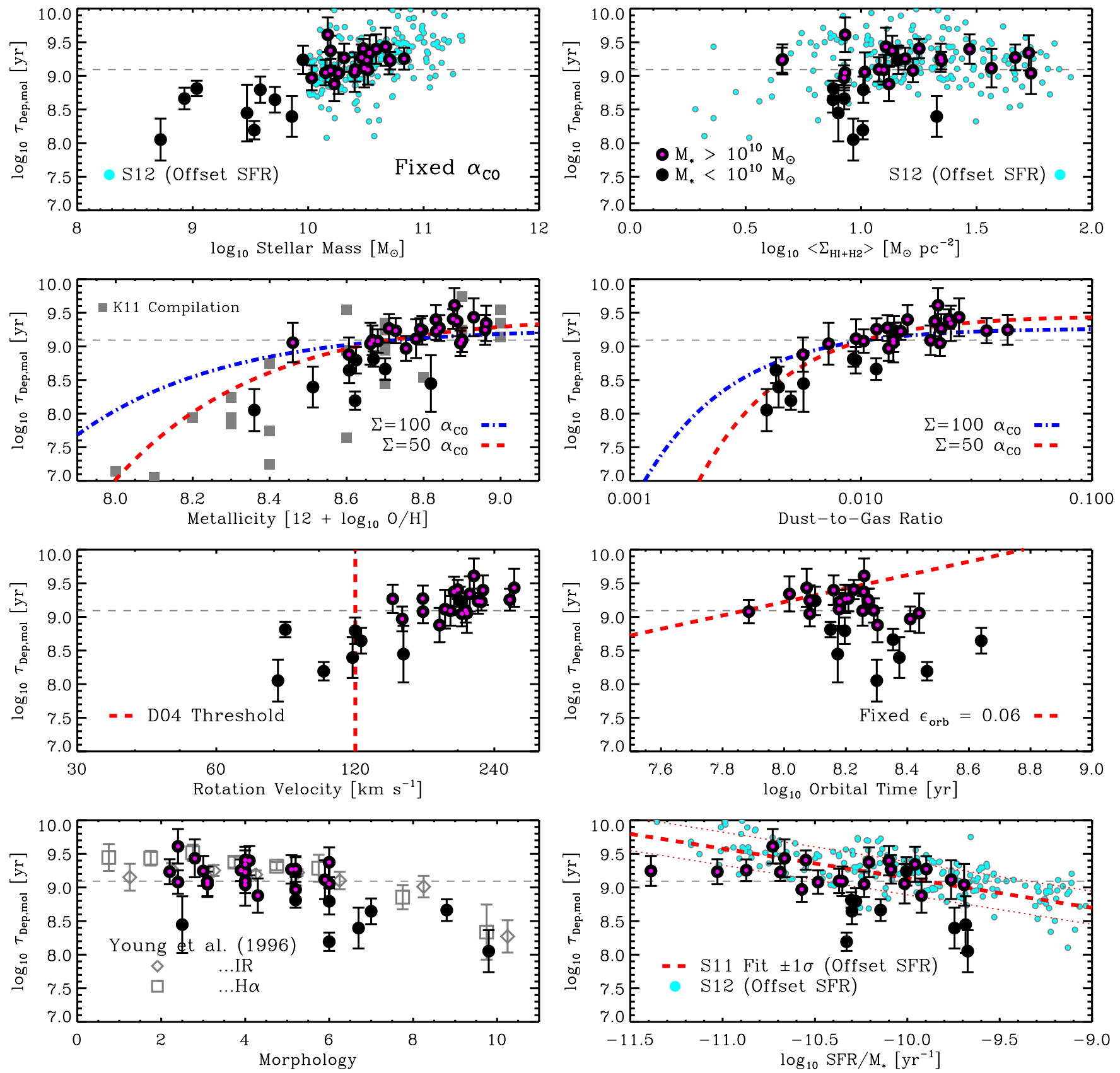

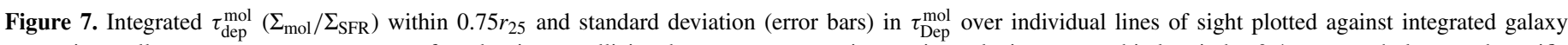
properties: stellar mass, average gas mass surface density, metallicity, dust-to-gas mass ratio, rotation velocity at $r_{25}$, orbital period at $0.4 r_{25}$, morphology, and specific SFR $\left(\left\langle\Sigma_{\mathrm{SFR}}\right\rangle /\left\langle\Sigma_{*}\right\rangle\right)$. Literature data appear in gray (Y96, K11, and S12 refer to Young et al. 1996; Krumholz et al. 2011; Saintonge et al. 2012), and fits or predictions appear as curves: Wolfire et al. (2010) conversion factors for $\Sigma=50$ and $100 M_{\odot} \mathrm{pc}^{-2}$, the rotation-velocity threshold associated with the suppression of dust lanes by Dalcanton et al. (2004, D04), a fixed efficiency per orbital period, and the $\tau_{\text {dep }}^{\text {mol }}$ vs. SSFR fit by Saintonge et al. (2011, S11). Low-mass, metal-poor, late-type galaxies show significantly lower $\tau_{\text {dep }}^{\text {mol }}$ (high SFR-to-CO ratios) than their massive, early-type counterparts, but these trends persist with smaller dynamic range even among massive galaxies (we mark galaxies with $M_{*}>10^{10} M_{\odot}$ using magenta dots in all panels).

edge-on disk galaxies. The existence of such dust lanes should indicate the presence of a dusty, dense ISM of the type that might host molecular material and star formation. Though Dalcanton et al. (2004) make no clear prediction for $\tau_{\mathrm{dep}}^{\mathrm{mol}}$ we note that there is a generally lower $\tau_{\text {dep }}^{\text {mol }}$ below the threshold than above; at the very least, the lack of abundant dust shielding may drive both observations. This contrasts with the finding of Watson et al. (2012), who do not find such a trend with circular velocity.

Table 6 quantifies the relationship between $\tau_{\mathrm{dep}}^{\mathrm{mol}}$ and these other quantities using the Spearman rank correlation coefficient. $\tau_{\text {dep }}^{\text {mol }}$ correlates with stellar mass, metallicity, dust-to-gas ratio, and rotation velocity at $>3 \sigma$ significance. Weaker $(\sim 2 \sigma)$ correlations link $\tau_{\text {dep }}^{\text {mol }}$ to gas surface density, orbital time, morphology, and sSFR. The trends with stellar mass and rotation velocity appear continuous, without clear thresholds. Indeed they still emerge, though at lower significance, even if we consider only high-mass galaxies.

The sense of the correlation with gas surface density is that we find the shortest depletion times for galaxies below $\left\langle\Sigma_{\mathrm{H} \text { I } \mathrm{H} 2}\right\rangle \sim 10 M_{\odot} \mathrm{pc}^{-2}$. These systems still yield the lowest 
Table 6

Rank Correlation of $\tau_{\mathrm{dep}}^{\mathrm{mol}}$ with Galaxy-average Properties

\begin{tabular}{lccr}
\hline \hline Quantity & Fixed $\alpha_{\mathrm{CO}}$ & $\Sigma=100 \alpha_{\mathrm{CO}}$ & $\Sigma=50 \alpha_{\mathrm{CO}}$ \\
\hline Stellar mass & $+0.72 \pm 0.18$ & $+0.47 \pm 0.19$ & $-0.07 \pm 0.19$ \\
$\ldots M_{*}>10^{10} M_{\odot}$ & $-0.37 \pm 0.20$ & $-0.15 \pm 0.24$ & $+0.02 \pm 0.22$ \\
$\left\langle\Sigma_{\mathrm{H} \text { I } 2}\right\rangle$ & $+0.40 \pm 0.19$ & $+0.64 \pm 0.18$ & $+0.49 \pm 0.20$ \\
$\ldots M_{*}>10^{10} M_{\odot}$ & $+0.19 \pm 0.20$ & $+0.60 \pm 0.22$ & $+0.60 \pm 0.25$ \\
Metallicity & $+0.73 \pm 0.21$ & $+0.44 \pm 0.19$ & $-0.14 \pm 0.19$ \\
$\ldots M_{*}>10^{10} M_{\odot}$ & $+0.59 \pm 0.20$ & $0.16 \pm 0.23$ & $-0.21 \pm 0.22$ \\
Dust-to-gas ratio & $+0.81 \pm 0.19$ & $+0.28 \pm 0.19$ & $-0.49 \pm 0.19$ \\
$\ldots M_{*}>10^{10} M_{\odot}$ & $+0.57 \pm 0.20$ & $-0.24 \pm 0.22$ & $-0.72 \pm 0.23$ \\
Rotation velocity & $+0.74 \pm 0.20$ & $+0.47 \pm 0.20$ & $-0.11 \pm 0.23$ \\
$\ldots M_{*}>10^{10} M_{\odot}$ & $-0.40 \pm 0.24$ & $+0.02 \pm 0.026$ & $-0.24 \pm 0.23$ \\
Orbital time & $-0.45 \pm 0.21$ & $-0.36 \pm 0.19$ & $-0.12 \pm 0.20$ \\
$\ldots M_{*}>10^{10} M_{\odot}$ & $-0.32 \pm 0.24$ & $-0.21 \pm 0.23$ & $-0.03 \pm 0.22$ \\
Morphology & $-0.49 \pm 0.19$ & $-0.30 \pm 0.19$ & $-0.03 \pm 0.20$ \\
$\ldots M_{*}>10^{10} M_{\odot}$ & $-0.16 \pm 0.23$ & $+0.03 \pm 0.23$ & $+0.16 \pm 0.22$ \\
SFR $/ M_{*}$ & $-0.38 \pm 0.19$ & $-0.06 \pm 0.19$ & $0.32 \pm 0.19$ \\
$\ldots M_{*}>10^{10} M_{\odot}$ & $-0.27 \pm 0.22$ & $+0.16 \pm 0.23$ & $0.34 \pm 0.25$ \\
\hline
\end{tabular}

Notes. Rank correlation coefficient relating the molecular gas depletion time, $\tau_{\mathrm{dep}}^{\mathrm{mol}}$, to galaxy-average properties. Quoted uncertainties report the $1 \sigma$ scatter of the correlation coefficient about 0 obtained by randomly repairing the data. The columns give results for different assumptions about the CO-to- $\mathrm{H}_{2}$ conversion factor. We report results for all galaxies and only high-mass galaxies, $M_{*}>10^{10} M_{\odot}$. The average molecular gas depletion time is strongly covariant with galaxy average properties. This covariance can be reduced but not removed by the application of a D/G-dependent $\mathrm{CO}$-to- $\mathrm{H}_{2}$ conversion factor.

$\tau_{\text {dep }}^{\text {mol }}$ regardless of our treatment of $\alpha_{\mathrm{CO}}$ (see below). In our sample, these low $\left\langle\Sigma_{\mathrm{H} \text { I } \mathrm{H} 2}\right\rangle$ hold more of their gas in the atomic phase than systems with high $\left\langle\Sigma_{\mathrm{H}+\mathrm{H} 2}\right\rangle$. Therefore, the trend we see amounts to longer depletion times in gas-rich, moleculedominated systems. However, note that our high $\left\langle\Sigma_{\mathrm{H}+\mathrm{H} 2}\right\rangle$ systems are gas-rich spirals. Our sample does not include truly extreme systems like the local U/LIRGs. Many of these systems have high gas surface densities but very low $\tau_{\text {dep }}^{\text {mol }}$ (e.g., Sanders \& Mirabel 1996), so that if they were added to Figure 7, they would not extend the trend that we see. We do see similar efficient starbursts within our targets (Section 4.4), but the effect remains mostly confined to the central parts of galaxies and does not propagate to these galaxy-scale measurements. This behavior may be somewhat evident in the Saintonge et al. (2012) sample, but not our data.

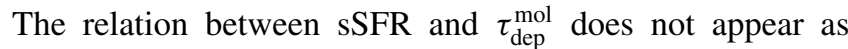
strong as other trends in our sample, contrary to the finding of Saintonge et al. (2011). This may simply reflect our sample's lack of massive early-type galaxies with low sSFR or U/LIRGs with high sSFR. Where we do overlap Saintonge et al. (2012), the agreement between the samples appears very good after our adjustment for different approaches to SFR estimation (Section 2). The most substantive observational disagreement between our results and those of Saintonge et al. (2011) is that we find $\tau_{\text {dep }}^{\text {mol }}$ to correlate with metallicity and dust-to-gas ratio, even for relatively high mass galaxies, while their tests revealed no such correlations.

Dust-to-gas Ratio and Conversion Factor. We emphasize that the correlations in Figure 7 relate apparent $\tau_{\mathrm{dep}}^{\mathrm{mol}}$ to integrated galaxy properties. The second row of Figure 7 shows strong trends in $\tau_{\text {dep }}^{\text {mol }}$ as a function of metallicity and dust-to-gas ratio, and these trends raise a fundamental issue regarding the interpretation of Figure 7. There is good evidence that the $\mathrm{CO}-$ to- $\mathrm{H}_{2}$ conversion factor increases with decreasing metallicity and dust-to-gas ratio (see references in Israel 1997; Leroy et al. 2011; Bolatto et al. 2011). Metallicity and dust-to-gas ratio vary with the stellar and dynamical mass of a galaxy, so that the correlations in Figure 7 and Table 6 may reflect either true variation in the efficiency with which molecular gas forms stars, variations in the $\mathrm{CO}-$ to- $\mathrm{H}_{2}$ conversion factor, or a mixture of the two.

The blue and red curves in the second row show the dependence of $\alpha_{\mathrm{CO}}$ on metallicity or dust-to-gas ratio for the " $\Sigma=100 "$ (blue) and " $\Sigma=50 "$ (red) models described in Section 2 assuming a fixed molecular gas depletion time. The third and fourth columns in Table 6 report the correlations between $\tau_{\mathrm{dep}}^{\mathrm{mol}}$ with global properties after application of these conversion factors. ${ }^{14}$ The red curve, representing the more extreme assumption that $\mathrm{CO}$ emission arises from low surface density $\left(\Sigma=50 M_{\odot} \mathrm{pc}^{-2}\right.$ ) clouds fits the dust-to-gas ratio trend well. Neither trend is steep enough to fit the metallicity data (left panel, second row), but given the large uncertainties in the metallicities (often several 0.1s of a dex, e.g., Moustakas et al. 2010), the " $\Sigma=50$ " curve is not a bad match.

Comparison of these two curves and the second row of Figure 7 highlights several technical points also found in the recent literature. First, the conversion factor needed to

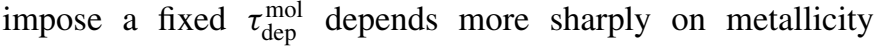
than the predictions from many theoretical models and some observational determinations (see plots in Krumholz et al. 2011; Schruba et al. 2012; Genzel et al. 2012). Second, the left and right panels of the second row do not perfectly agree. The dustto-gas ratio derived from Spitzer observations of low-metallicity regions appears somewhat lower than one would predict from a linear scaling of their metallicity (Muñoz-Mateos et al. 2009). Because both the observed and predicted trends flatten at high $\mathrm{D} / \mathrm{G}$ and high metallicities, the zero points (solar values) used to compute the theoretical curves might be adjusted to yield better agreement between metallicity and D/G or to better match one theoretical curve or the other to the data. That is, given the uncertainties in the absolute measurements of both metallicity and D/G, one can "slide" the relative positions of the theoretical curves and the $\mathrm{D} / \mathrm{G}$ and metallicity determinations left and right. As a result, the differences between the " $\Sigma=50$ " and " $\Sigma=100$ " curves should not be overemphasized.

These details aside, the key questions are: to what degree can conversion factor variations explain the global trends seen in Figure 7 and are the adopted conversion factors reasonable? In Figure 8 we repeat Figure 7 but adopt our " $\Sigma=100$ " conversion factor rather than fixed $\alpha_{\mathrm{CO}}$. Many correlations of $\tau_{\mathrm{dep}}^{\mathrm{mol}}$ with local properties appear weaker with the " $\Sigma=100 " \alpha_{\mathrm{CO}}$ than with a fixed conversion factor. The more extreme " $\Sigma=50$ " case removes almost all variation with stellar mass and metallicity, but reverses the trend with dust-to-gas ratio. Thus, the " $\Sigma=50$ " may represent the simplest single explanation for the bulk of the variation in Figure 7. " $\Sigma=100$ " corresponds to a conservative $\alpha_{\mathrm{CO}}$ estimate that reflects present conventional wisdom. It lessens the strength of many correlations, but (marginally) significant variations in $\tau_{\mathrm{dep}}^{\mathrm{mol}}$ with other properties still exist when using this conversion factor.

\subsubsection{Conversion Factors and ISM Structure}

Both of our conversion factors assume a simple, universal structure for the molecular ISM beneath our kpc resolution.

\footnotetext{
14 Note that the conversion factors are calculated from the dust-to-gas ratio profiles and then applied to the $\mathrm{CO}$ maps to derive the integrated $\mathrm{H}_{2}$ masses and that these conversion factors include central $\alpha_{\mathrm{CO}}$ depressions, so that the mapping of conversion factor to the average dust-to-gas ratio is not perfect.
} 

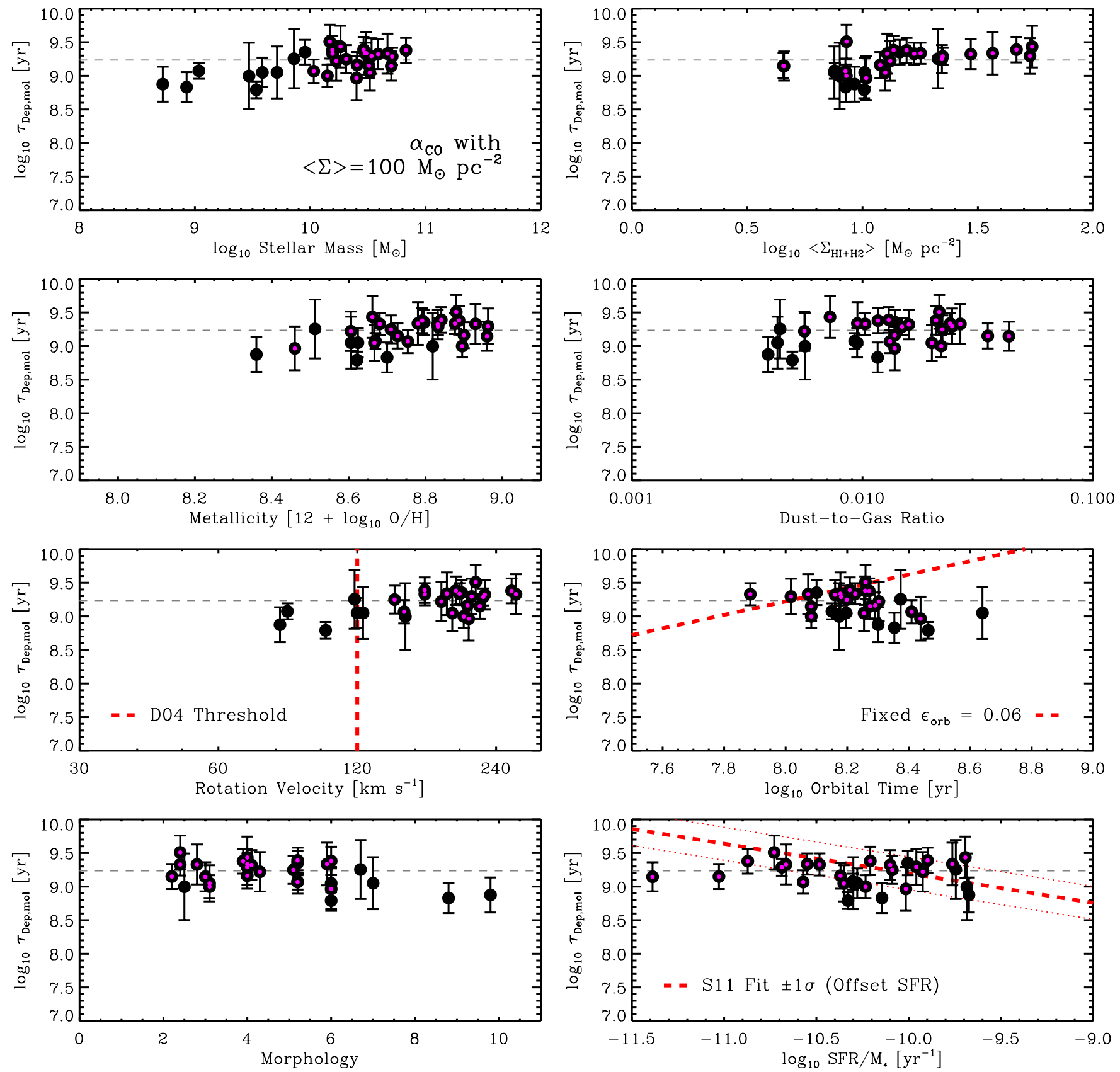

Figure 8. Same as Figure 7 but after application of our variable " $\Sigma=100$ " CO-to- $\mathrm{H}_{2}$ conversion factor. Most of the galaxy-scale variations in $\tau_{\mathrm{dep}}^{\mathrm{mol}}$ visible in Figure 7 can largely be explained by this dust-to-gas ratio-dependent conversion factor, though significant galaxy-to-galaxy scatter remains. A dashed horizontal line in each panel shows the median $\tau_{\text {dep }}^{\text {mol }}$ across our sample, and we reproduce several comparison lines from Figure 7.

In actuality, the typical surface density of GMCs, the universality of this value, and the balance of GMCs and more diffuse molecular material remain poorly constrained by observations. Much previous work places typical surface densities of Milky Way GMCs at $\sim 150 M_{\odot} \mathrm{pc}^{-2}$ (Solomon et al. 1987; RomanDuval et al. 2010). High-resolution observations of the inner parts of nearby spirals (NGC 5194, NGC 6946) yield similar surface densities (Donovan Meyer et al. 2012; Hughes et al. 2013; Colombo et al., in preparation). Other studies find lower surface densities, even in the same systems: e.g., studies of the Milky Way ring by Heyer et al. (2009), the disk of NGC 6946 (Rebolledo et al. 2012), the LMC (Fukui et al. 2008; Hughes et al. 2010; Wong et al. 2011), or a sample of Local Group galaxies (Bolatto et al. 2008) all find typical
GMC surface densities $\Sigma \sim 50-100 M_{\odot} \mathrm{pc}^{-2}$. Some of these differences may be attributed to methodology, but Hughes et al. (2013) decisively demonstrate that the structure of the molecular ISM does vary substantially with environment via a carefully matched comparison M51, M33, and the LMC. They show substantial differences in the probability distribution function of $\mathrm{CO}$ emission at $\sim 50 \mathrm{pc}$ resolution both within and among these galaxies.

Given these uncertainties, the best way to read the " $\Sigma=50$ " conversion factor is a case where $\alpha_{\mathrm{CO}}$ depends sharply on the dust-to-gas ratio because most $\mathrm{CO}$ emission comes from lowextinction lines of sight, $A_{V} \sim 1-2$ at Milky Way dust-to-gas ratios. In addition to the mixed evidence on surface densities of whole populations, maps of very local clouds (as in Heiderman 
et al. 2010; Lada et al. 2010) do tend to find significant mass in low- $A_{V}$ components. Furthermore, the contribution of diffuse sight lines (e.g., those studied by Liszt et al. 2010) to the integrated $\mathrm{CO}$ emission from a large part of a galaxy remains poorly known, but may easily be several tens of percent in the Milky Way (H. Liszt 2012, private communication).

A detailed investigation of GMC structure is beyond the scope of this paper. The key points are the following: (1) the basic structure of the molecular ISM remains uncertain at a level that significantly affects our interpretation of integrated CO emission, (2) the structure of the molecular ISM does vary substantially with environment, and (3) observations do appear to admit the possibility of substantial $\mathrm{CO}$ emission from low$A_{V}$ lines of sight or unbound clouds. Given these caveats, we must view our adopted conversion factors as more schematic than exact, so that subtle differences between the " $\Sigma=50 "$ and " $\Sigma=100 "$ case should not be over-interpreted. We can say from Table 6 and Figures 7 and 8 that the conversion factor can explain

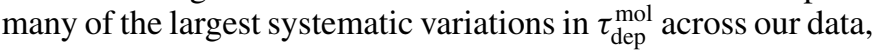
but probably not all of them. High-mass, high surface density galaxies probably are less efficient at forming stars from their molecular reservoir (i.e., high $\tau_{\text {dep }}^{\mathrm{mol}}$ ) than low-mass galaxies.

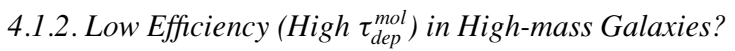

Our best guess is that weak correlation exists relating $\tau_{\mathrm{dep}}^{\mathrm{mol}}$ to galaxy mass and average surface density, reflecting a factor of $\sim 2$ increase in $\tau_{\text {dep }}^{\text {mol }}$ moving from low $\left(M_{*}<10^{10} M_{\odot}\right)$ to high $\left(M_{*}>10^{10} M_{\odot}\right)$ mass galaxies. Several natural explanations exist for such trends, and a combination of these are almost certainly at play. Suppression of star formation in molecular gas by dynamical effects appears evident from high-resolution observations of M51 (Meidt et al. 2013) and in some barred spiral galaxies (see discussion in Jogee et al. 2005). These largescale dynamical effects may suppress the ability of molecular gas to collapse into gravitationally bound, star-forming clouds.

This represents a subset of a more general effect: in regions of high pressure and high gas surface density, the ISM becomes increasingly molecular (Blitz \& Rosolowsky 2006; L08). A diffuse molecular ISM, meaning unbound material and the lowdensity outskirts of bound clouds, may represent a reservoir of non-star-forming molecular gas (though note that even the bulk of bound, molecular material does not directly participate in star formation; see references in Lada et al. 2012). In low-mass, low molecular fraction galaxies such gas, if present, will often be in the atomic phase. Higher mass galaxies will have both a higher molecular fraction and stronger dynamical effects such as shear and streaming motions working at suppressing the creation of bound gas. See Saintonge et al. (2012) for a discussion of such effects as drivers for the correlations between $\tau_{\mathrm{dep}}^{\mathrm{mol}}$ mass and morphology observed by the COLDGASS survey.

This schematic picture leads to several predictions and is clouded by several complications. Both a prediction and a complication is that in this sketch, the physical state of molecular gas varies systematically as a function of galaxy mass. This should manifest via several direction observables such as the $\mathrm{CO}$-to-HCN or CO-to-HCO+ ratio, which will trace the fraction of dense molecular gas, the ratio of ${ }^{13} \mathrm{CO}-$ to- $^{12} \mathrm{CO}$, tracing the optical depth of the gas, and the ratio of $\mathrm{CO}$ rotational transitions, which trace a complex combination of density, temperature, and deviation from local thermodynamic equilibrium. An immediate complication from such variations is that changes in internal conditions propagate to $\alpha_{\mathrm{CO}}$ variations that are distinct from the dust-shielding effects accounted for in our adopted conversion factors (e.g., see discussion in Maloney \& Black 1988). A second, weak prediction would be a general increase in $\tau_{\mathrm{dep}}^{\mathrm{mol}}$ with increasing molecular fraction, at least up to a certain extent - starbursts exhibit both low $\tau_{\text {dep }}^{\text {mol }}$ and high molecular fractions. Systematic trends in $\tau_{\text {dep }}^{\text {mol }}$ with molecular fraction were not immediately clear in L08; we search for them in our present data set in the next section. A similar mixed picture arises from dynamical effects: one might expect to see high molecular fractions but low efficiencies in regions where shear or streaming motions suppress bound cloud formation. However, Foyle et al. (2010) found little or no evidence for $\tau_{\mathrm{dep}}^{\mathrm{mol}}$ variations between spiral arms and the surrounding material. Again, a similar case of competing effects comes into play, given that spiral arms are also invoked as a mechanism to collect inefficient, low $\tau_{\mathrm{dep}}^{\mathrm{mol}}$ gas into bound, high-efficiency objects (e.g., Tan 2000; Koda et al. 2009). Similarly, bars may both suppress inflowing gas and concentrate material into nuclear starbursts (e.g., Sakamoto et al. 1999; Jogee et al. 2005; Sheth et al. 2005).

Though competing effects cloud a simple interpretation of the data, the path forward here remains relatively clear: systematic measurements of the internal conditions in the molecular gas represent a critical next step, and our knowledge of the $\mathrm{CO}-\mathrm{to}-\mathrm{H}_{2}$ conversion factor must improve to further

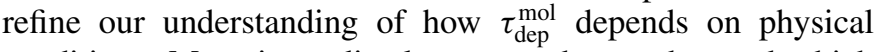
conditions. More immediately, we need to understand which local conditions drive galaxy-averaged trends seen in this section. Finally, we emphasize that while these details are essential to a complete understanding of star formation in galaxies, they appear less critical to explain Figure 7 than the CO-to- $\mathrm{H}_{2}$ conversion factor and represent, in some sense, a factor of $\sim 2$ level correction to a constant $\tau_{\mathrm{dep}}^{\mathrm{mol}}$.

\subsection{Local Variations in $\tau_{d e p}^{m o l}$}

HERACLES resolves our targets, so that we can investigate the dependence of the local $\tau_{\text {dep }}^{\text {mol }}$ on local conditions. As Figure 7 shows, galaxy-averaged properties have a high degree of covariance. Examining local conditions may help break this degeneracy. Figure 9 plots the data density (blue contours) for fixed $\alpha_{\mathrm{CO}}$ as a function of local (kpc-scale) conditions: stellar mass surface density, $\Sigma_{*}$ (top left), $\mathrm{H}_{2}$ surface density, $\Sigma_{\text {mol }}$ (top right), dust-to-gas ratio (bottom left), and orbital time (bottom right). In red we show the median and standard deviation for data binned by the abscissa. These medians incorporate the fraction of upper and lower limits in the bin, which appear as percentages running along the top and bottom of each plot. The gray region in each plot indicates where the percentage of limits exceeds $\sim 30 \%$, indicating significant issues with completeness.

Table 7 reports rank correlations between $\tau_{\text {dep }}^{\text {mol }}$ and local physical conditions. We restrict these calculations to the region of approximate completeness. This minimizes biases, but prevents us from probing very low surface densities. Binning and profile work will allow us to extend these analyses in future work (Schruba et al. 2011). Figure 9 shows little systematic variation $\tau_{\text {dep }}^{\text {mol }}$ as a function of $\Sigma_{*}$ and $\Sigma_{\text {mol }}$, only a weak tendency to have shorter $\tau_{\mathrm{dep}}^{\mathrm{mol}}$ at the highest surface densities (for constant $\alpha_{\mathrm{CO}}$ ). These trends have the opposite sense of those expected based on our examination of galaxy average properties, where highmass, high $\Sigma_{\mathrm{H} \mathrm{I}+\mathrm{H} 2}$ systems showed longer depletion times than low-mass systems. 

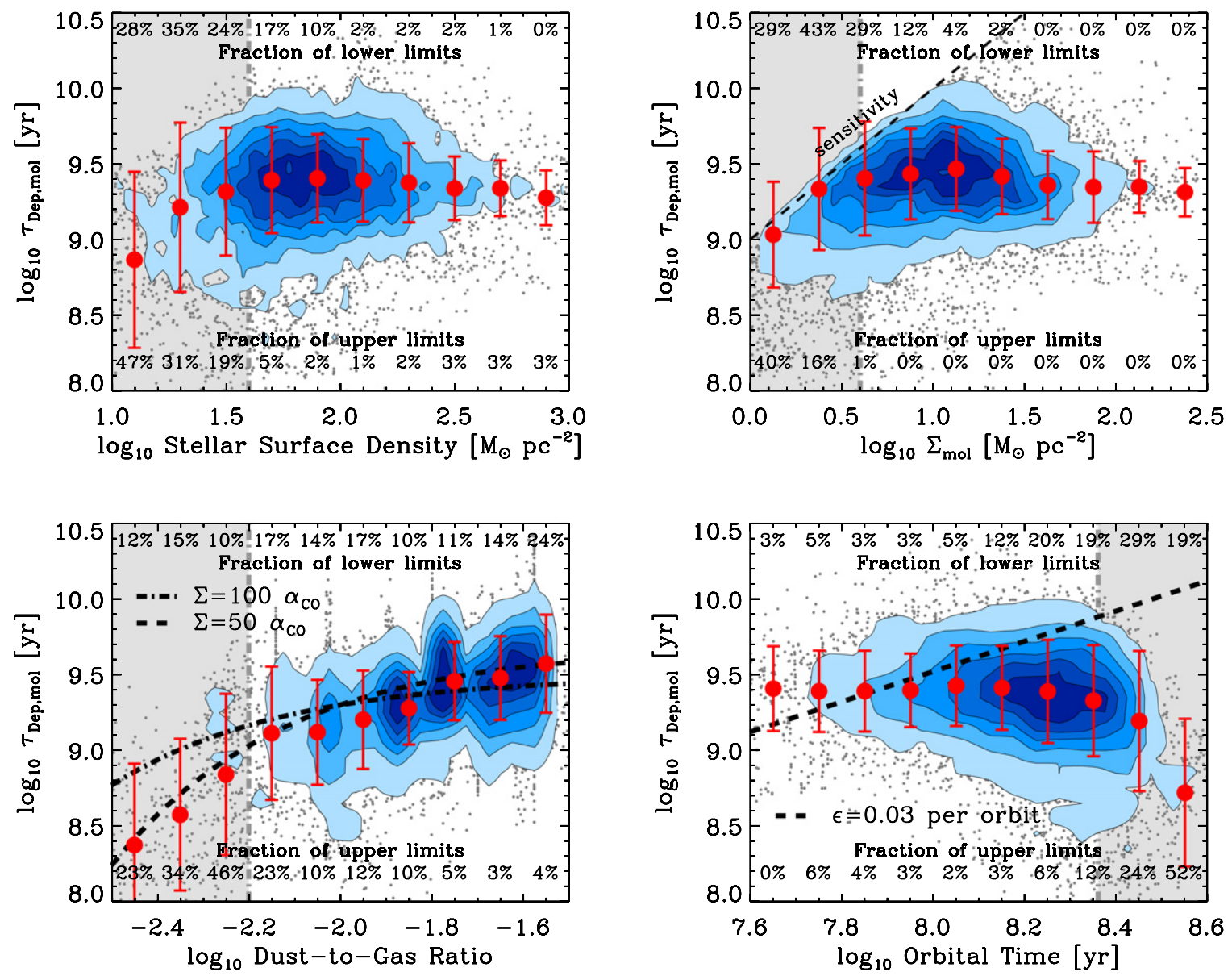

Figure 9. Local $\tau_{\mathrm{dep}}^{\mathrm{mol}}=\Sigma_{\mathrm{mol}} / \Sigma_{\mathrm{SFR}}$ for fixed $\alpha_{\mathrm{CO}}$ as a function stellar mass surface density (top left), $\mathrm{H}_{2}$ surface density (top right), dust-to-gas ratio (bottom left), and orbital period (bottom right) for a fixed $\alpha_{\mathrm{CO}}$. Gray points show individual lines of sight, and shaded blue contours show density of data. Red points show the median (including relevant upper and lower limits) and standard deviation in $\log _{10} \tau_{\mathrm{dep}}^{\mathrm{mol}}$ binned by the $x$-axis. Percentages along the top and bottom indicate the fraction of lower and upper limits in each bin. Gray regions indicate where $\gtrsim 30 \%$ of our $\tau_{\mathrm{dep}}^{\mathrm{mol}}$ estimates are limits so that completeness represents a serious concern. The dashed curve in the lower left panel shows the expected relation for an $\alpha_{\mathrm{CO}}$-dependent $\mathrm{D} / \mathrm{G}$, and the dashed line in the lower right panel shows the expectation for a fixed fraction of molecular converted to stars per orbital period.

Table 7

Rank Correlation of $\tau_{\mathrm{dep}}^{\mathrm{mol}}$ with Local Conditions

\begin{tabular}{lccc}
\hline \hline Quantity & Fixed $\alpha_{\mathrm{CO}}$ & $\Sigma=100 \alpha_{\mathrm{CO}}$ & $\Sigma=50 \alpha_{\mathrm{CO}}$ \\
\hline$\Sigma_{*}$ & $-0.03 \pm 0.02$ & $-0.01 \pm 0.02$ & $-0.08 \pm 0.02$ \\
$\Sigma_{\text {mol }}$ & $-0.06 \pm 0.02$ & $-0.04 \pm 0.02$ & $+0.15 \pm 0.02$ \\
$f_{\text {mol }}=\Sigma_{\text {mol }} /\left(\Sigma_{\text {mol }}+\Sigma_{\mathrm{HI}}\right)$ & $+0.04 \pm 0.03$ & $+0.01 \pm 0.02$ & $+0.04 \pm 0.02$ \\
Dust-to-gas ratio & $+0.39 \pm 0.02$ & $+0.09 \pm 0.02$ & $-0.23 \pm 0.02$ \\
Orbital time & $-0.05 \pm 0.02$ & $+0.07 \pm 0.02$ & $+0.12 \pm 0.02$ \\
Galactocentric radius $\left(r / r_{25}\right)$ & $-0.05 \pm 0.02$ & $+0.09 \pm 0.02$ & $+0.13 \pm 0.02$
\end{tabular}

Notes. Rank correlation between $\tau_{\text {dep }}^{\text {mol }}$ for individual kpc-resolution lines of sight and local conditions for regions where we are reasonably complete. Uncertainties give the $1 \sigma$ scatter of the rank correlation about zero obtained when randomly repairing the data and accounting for an oversampling factor of four. The three columns report results for different assumed $\mathrm{CO}-\mathrm{to}_{\mathrm{H}} \mathrm{H}_{2}$ conversion factors (Section 2). We report results only for the range over which $\lesssim 30 \%$ of our data are limits. This is approximately $\Sigma_{*}>40 M_{\odot} \mathrm{pc}^{-2}$, $\Sigma_{\text {mol }}>4 M_{\odot} \mathrm{pc}^{-2}, f_{\text {mol }}>0.5, \mathrm{D} / \mathrm{G}>0.006, \tau_{\text {orb }}<0.23 \mathrm{Gyr}$, and $r_{\text {gal }}<0.55 r_{25}$. A strong correlation with the dust-to-gas ratio is evident for fixed $\alpha_{\mathrm{CO}}$. For a variable $\alpha_{\mathrm{CO}}$ weak correlations.

We observe a more significant relation between $\tau_{\text {dep }}^{\text {mol }}$ and $\mathrm{D} / \mathrm{G}$, one that becomes even stronger when limits are factored in. The functional form of the local trend in $\tau_{\text {Dep }}^{\text {mol }}$ as a function of $D / G$ closely matches the trend seen for whole galaxies. To some degree this reflects the fact that the $\mathrm{D} / \mathrm{G}$, unlike $\Sigma_{*}$ or $\Sigma_{\mathrm{mol}}$, remains relatively constant across the disks of many of our targets (see Appendix). By contrast even a galaxy with very high total $M_{*}$ or $M_{\mathrm{mol}}$ will have a wide range of $\Sigma_{*}$ and $\Sigma_{\mathrm{mol}}$.

Figure 10 shows $\tau_{\mathrm{dep}}^{\mathrm{mol}}$ as a function of the same local conditions after applying our " $\Sigma=100$ " conversion factor. The correspondence between $\tau_{\text {dep }}^{\text {mol }}$ and $\mathrm{D} / \mathrm{G}$ mostly vanishes if we adopt this conversion factor. Meanwhile the weak correlations of $\tau_{\text {dep }}^{\text {mol }}$ with $\Sigma_{*}$ and $\Sigma_{\text {mol }}$ remain weak.

For fixed $\alpha_{\mathrm{CO}}$, Figure 9 does not support the idea that a fixed fraction of gas is converted to stars each orbital time. In the bottom right panel the red bins do not match the dashed line, which shows a fixed fraction of gas converted to stars per orbital time. Adopting the " $\Sigma=100$ " conversion factor changes this picture somewhat, as the central $\alpha_{\mathrm{CO}}$ depressions lead to shorter $\tau_{\text {dep }}^{\text {mol }}$ in regions with short orbital times. With the " $\Sigma=100$ " $\alpha_{\mathrm{CO}}$, a fixed efficiency per orbital time becomes a reasonable description of the data below $\tau_{\text {orb }} \sim 100$ Myr. Most of our data have longer $\tau_{\text {orb }}$ than this, so that this statement relates mostly to the inner parts of galaxies. Regardless of $\alpha_{\mathrm{CO}}$, a fixed efficiency per orbital time does not appear to describe most of our data. Instead, this may be a reasonable description of integrated galaxies across a wide range of luminosities (Kennicutt 1998a; 

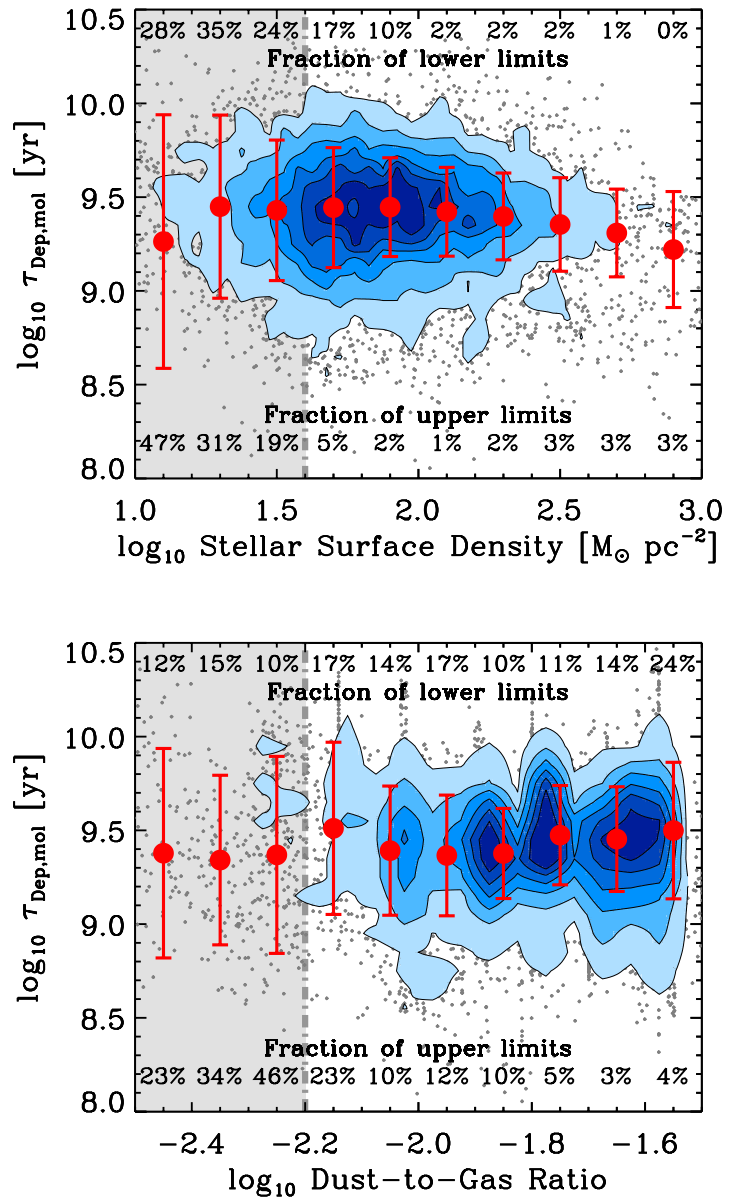
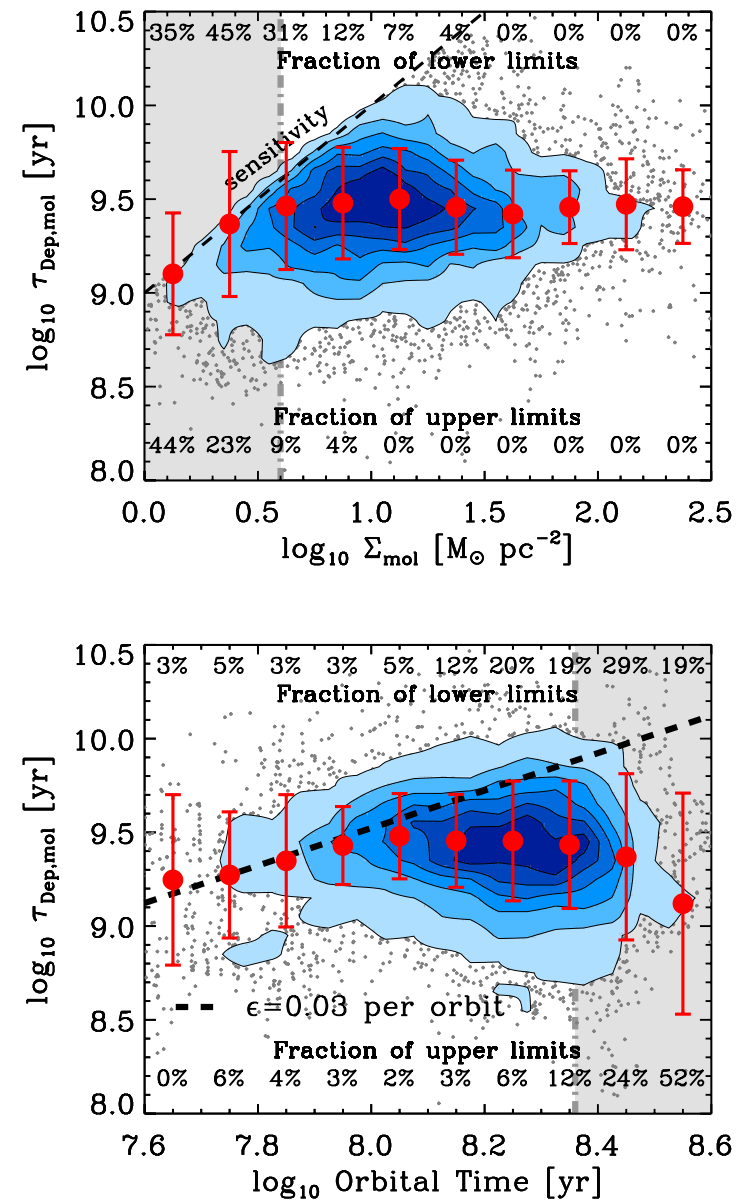

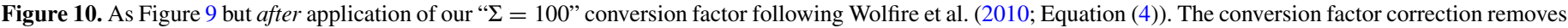
the strongest visible trend in the data.

Daddi et al. 2010; Genzel et al. 2010) or relevant to inner regions where the orbital timescale becomes comparable to the internal dynamical time of bound clouds. Treatment of $\alpha_{\mathrm{CO}}$ makes a large difference to the results in this plot, highlighting the need for improved constraints in the inner disks of galaxies where variations in physical condition presumably dominate $\alpha_{\mathrm{CO}}$ variations.

In Section 4.1 we discuss the idea that the conversion of the diffuse, unbound ISM from atomic to molecular may lead to the high $\tau_{\mathrm{dep}}^{\mathrm{mol}}$ found in massive galaxies. The local drivers of such trends are not immediately obvious from Figure 9. In Figure 11 we directly plot $\tau_{\mathrm{dep}}^{\mathrm{mol}}$ as a function of the local molecular fraction, $f_{\mathrm{mol}}=\Sigma_{\mathrm{mol}} /\left(\Sigma_{\mathrm{mol}}+\Sigma_{\mathrm{H}}\right)$. For $f_{\mathrm{mol}}>0.5$ molecular gas dominates the ISM mass budget. We do not know whether this molecular gas is organized into bound, starforming clouds or diffuse, inert material, but if high $f_{\text {mol }}$ does correspond to a higher fraction of diffuse molecular material, we might expect to observe a general increase in $\tau_{\text {dep }}^{\text {mol }}$ as $f_{\text {mol }}$ increases. As Figure 11 shows, our completeness severely limits this calculation, restricting us to $f_{\text {mol }} \gtrsim 0.5$. Above this value regardless of how we treat $\alpha_{\mathrm{CO}}$ we find little or no correlation of $\tau_{\mathrm{dep}}^{\mathrm{mol}}$ with $f_{\mathrm{mol}}$.

Comparison of $\tau_{\mathrm{dep}}^{\mathrm{mol}}$ to local conditions thus reveals the same strong trend with $\mathrm{D} / \mathrm{G}$ observed for galaxy-average properties but only weak trends with other parameters, including the molecular fraction. We observe a suggestion of decreased $\tau_{\text {dep }}^{\text {mol }}$ at high $\Sigma_{*}$ or high $\Sigma_{\mathrm{mol}}$, and after applying depressed $\alpha_{\mathrm{CO}}$ in galaxy centers, we find a weak correlation of orbital time and

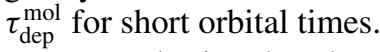

We emphasize that these represent our broad-brush results. Our database will allow deeper exploration via detailed analysis of individual galaxies, deep profiles, varying weighting and normalization, and stacking (Schruba et al. 2011). Kinematic analysis (e.g., Tan 2000) and the inclusion of outer disks (e.g., Bigiel et al. 2010) should yield the lever arms to better understand the impact of local conditions on star formation in molecular gas. Indeed, as we discuss in the next section, significant peripheral evidence points to the existence of significant environmental dependencies of $\tau_{\mathrm{dep}}^{\mathrm{mol}}$. Such effects are not immediately evident from the simple tests that we carry out here, however. Beyond the clear correlation of $\tau_{\text {dep }}^{\mathrm{mol}}$ with $\mathrm{D} / \mathrm{G}$, which we interpret as likely due to conversion factor effects, the absence of "smoking gun" correlations represents the main first-order result of this section.

\subsection{Spatial Correlation of $\tau_{d e p}^{m o l}$}

We have examined the explicit dependence of $\tau_{\mathrm{dep}}^{\mathrm{mol}}$ on various host galaxy properties and local physical conditions. We find many systematic dependencies on host galaxy, but the picture relating $\tau_{\text {dep }}^{\text {mol }}$ to local conditions remains more ambiguous. Considering the scatter in $\tau_{\text {dep }}^{\text {mol }}$ as a function of scale offers another way to approach this problem. For star formation uncorrelated on the scale of individual regions in a twodimensional disk, there is a clear analytic expectation for the 

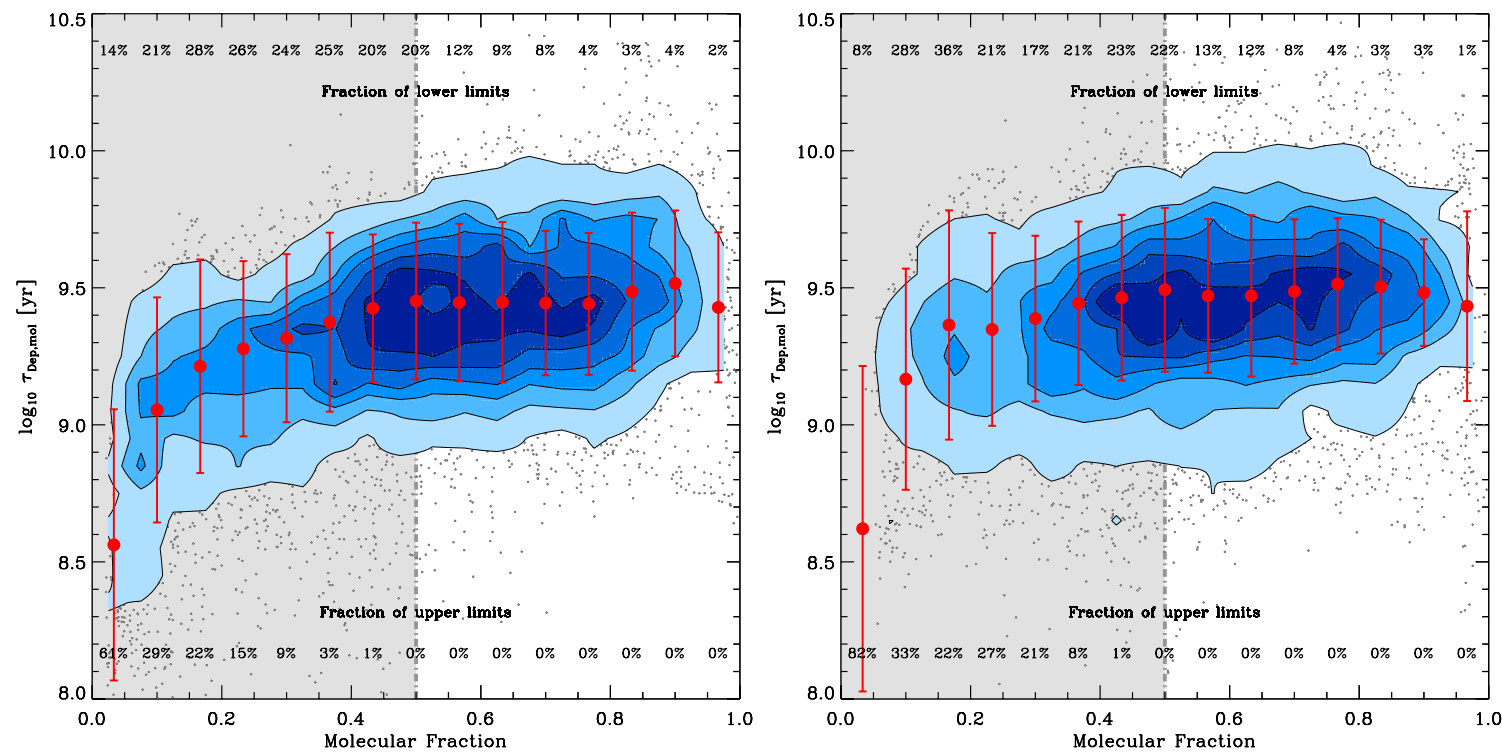

Figure 11. As Figure 9 but showing the molecular gas depletion time, $\tau_{\text {dep }}^{\text {mol }}$, as a function of the molecular fraction for (left) a fixed $\alpha_{\mathrm{CO}}$ and (right) our " $\Sigma=$ $100 " \alpha_{\mathrm{CO}}$.

functional form of this averaging. Deviations from this scaling can reveal the degree to which adjacent regions share the same $\tau_{\text {dep }}^{\text {mol }}$ or at least appear synchronized.

At small ( $\sim 10-50$ pc) scales observations of the Milky Way and the nearest galaxies resolve $\Sigma_{\mathrm{SFR}}$ and $\Sigma_{\text {mol }}$ into discrete star-forming regions, clouds, and clusters. These individual elements have distinct ages and evolutionary sequences (e.g., Kawamura et al. 2009; Fukui \& Kawamura 2010) so that the ratio of molecular gas to stars in a region and the emission of SFR tracers both evolve as a function of time (see discussion and plots in L12). As a result, scaling relations between SFR and $\mathrm{H}_{2}$, which capture the time-averaged relation between gas and star formation, emerge only after averaging together many distinct regions (see Schruba et al. 2010; Feldmann et al. 2011).

In a disk with fixed $\tau_{\text {dep }}^{\text {mol }}$ if these individual regions form stars independently, then we will expect the scatter in $\tau_{\text {dep }}^{\text {mol }}$ over part of the galaxy to go as $\sqrt{N^{-1}}$, where $N$ is the number of star-forming regions in that part of the galaxy. For a region of extent $l$ in a smooth disk, $N \propto l^{2}$, so that the expectation for the "uncorrelated case" is $\sigma \propto l^{-1}$.

Deviations from this scaling will emerge if $\tau_{\text {dep }}^{\text {mol }}$ varies systematically on large scales across the disk. In that case a high (or low) $\tau_{\mathrm{dep}}^{\mathrm{mol}}$ in one region is likely to be reflected with a similar $\tau_{\mathrm{dep}}^{\mathrm{mol}}$ in the adjacent regions. Thus, if $\tau_{\mathrm{dep}}^{\mathrm{mol}}$ is correlated between two adjacent regions, we expect a weaker dependence of the scatter in $\tau_{\text {dep }}^{\text {mol }}$ on scale. We would expect this to occur

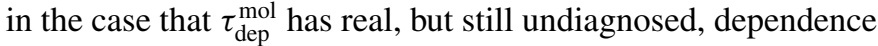
on local physical conditions. Moreover, large-scale dynamical effects like spiral density waves, bars, and supernova explosions may synchronize the star formation process on scales larger than a single cloud.

With $\sim \mathrm{kpc}$ resolution, HERACLES offers limited ability to measure the scale dependence of scatter over a large dynamic range, but we have identified a subset of large, nearby galaxies where we can measure the scatter in $\tau_{\text {dep }}^{\text {mol }}$ at linear resolutions from 0.6 to $2.4 \mathrm{kpc}$. These are labeled as our "multiscale" sample in Table 1. We use this sample to measure the scatter in $\log _{10} \tau_{\mathrm{dep}}^{\mathrm{mol}}$ as a function of linear resolution. To do this, we convolve each galaxy in our "multiscale" sample (Table 1) to have linear resolution $0.6-2.4 \mathrm{kpc}$ and measure the rms scatter

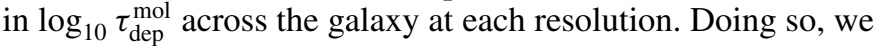
make no correction for inclination, so that this exercise consists of placing targets at larger and larger distances.

Figure 12 plots the results of this exercise for each resolved

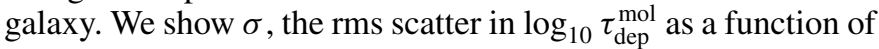
spatial resolution. The figure shows results calculated using the " $\Sigma=100 " \alpha_{\mathrm{CO}}$, which removes a significant internal gradient

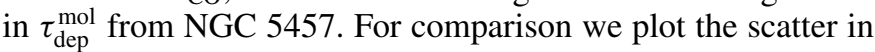
$\tau_{\text {dep }}^{\text {mol }}$ measured at high resolution in M33 ${ }^{15}$ by Schruba et al. (2010, stars) and M51 (Blanc et al. 2009, diamonds).

Figure 12 shows a steady increase in scatter with improving linear resolution. We characterize this scale dependence of the scatter via a power-law,

$$
\sigma(l)=\sigma_{600}\left(\frac{l}{600 \mathrm{pc}}\right)^{-\beta}
$$

where $l$ represents the spatial resolution, $\sigma_{600}$ is the scatter in $\tau_{\text {dep }}^{\text {mol }}$ at 600 pc resolution, and the power-law index $\beta$ measures the rate at which changing the resolution changes the measured scatter in $\tau_{\mathrm{dep}}^{\mathrm{mol}}$. We report the best-fit $\beta$ for each multiscale target in Table 8. As described above, we expect $\beta=1$ for uncorrelated star formation in a disk.

In most cases the best-fit averaging index, $\beta$, is $\sim 0.5$, significantly less than the $\beta=1$ expected for uncorrelated, fixed-efficiency star formation. Based on the previous sections,

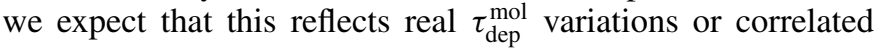
systematic uncertainties in our physical parameter estimation (e.g., undiagnosed $\alpha_{\mathrm{CO}}$ variations). That is, this is another way to see the subtle but real systematic variations in $\tau_{\text {dep }}^{\text {mol }}$ considered in the last two sections. Alternatively, Figure 12 and Table 8 could reflect a high degree of synchronization, with adjacent regions likely to be at the same stage of the star formation process and thus showing similar ratios of star formation tracers to $\mathrm{CO}$ emission. Either synchronization or real $\tau_{\mathrm{dep}}^{\mathrm{mol}}$ variations 15 We infer the scatter from their measurements of CO-to- $\mathrm{H} \alpha+24 \mu$ m near $\mathrm{H} \alpha$
peaks and $\mathrm{CO}$ peaks. 


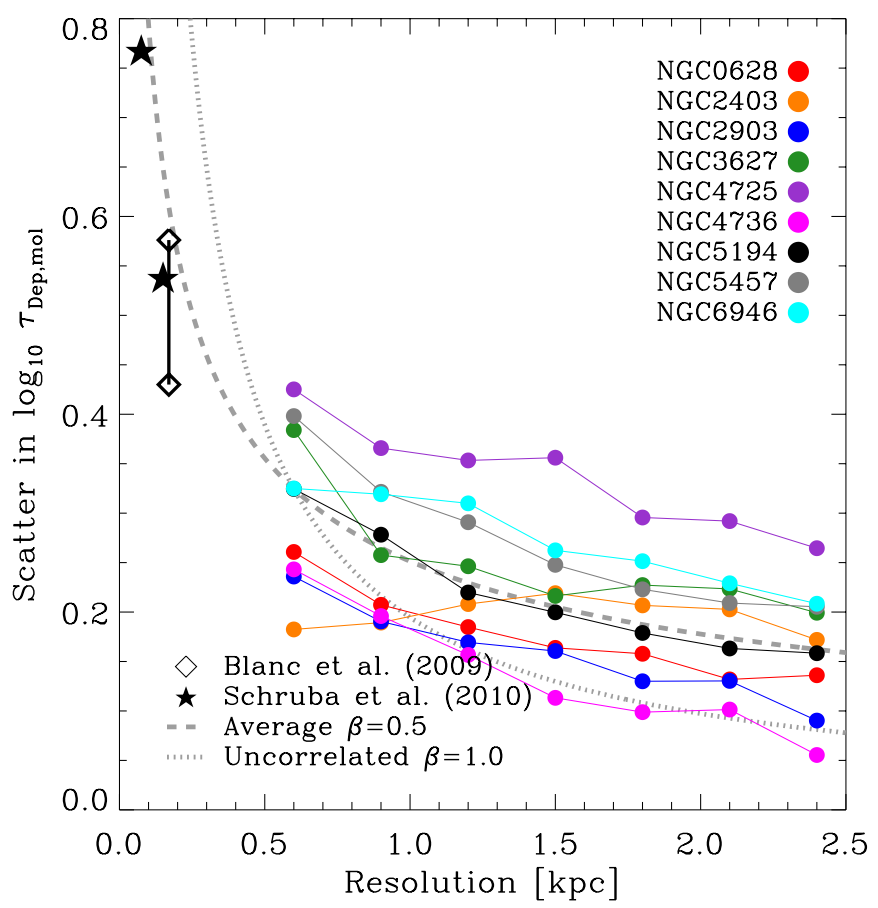

Figure 12. Evidence for spatial correlation among $\tau_{\text {dep }}^{\text {mol }}$ within galaxies. We plot rms scatter in $\log _{10} \tau_{\mathrm{dep}}^{\text {mol }}$ (y-axis) as a function of spatial resolution ( $x$-axis) for a subset of nearby, large galaxies. The scatter in $\tau_{\mathrm{dep}}^{\mathrm{mol}}$ changes more slowly as a function of resolution than one would expect averaging uncorrelated data. Extrapolating the trend that we observe to much higher resolution yields reasonable consistency with (scarce) high-resolution literature data (Blanc et al. 2009; Schruba et al. 2010). We illustrate the median trend, $\beta=0.5$, while $\beta=1$ would be expected for a disk filled with uncorrelated star-forming regions. This

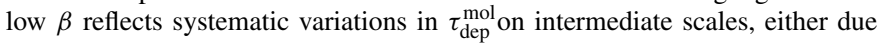
to synchronization of star formation on intermediate scales (e.g., see Feldmann et al. 2011) or systematic but still undiagnosed environment dependencies of the efficiency of star formation.

might be achieved by dynamical phenomena at small scales, and systematic efficiency variations may arise from dependence on local conditions that we have yet to identify.

This weak $(\beta \sim 0.5)$ scale dependence agrees with predictions based on numerical simulations by Feldmann et al. (2011), who discuss $\beta$ in terms of dimensionality. As an example, $\beta=0.5$ would be consistent with the degree of correlation expected by spiral arms or any other phenomenon that synchronizes star formation along one dimension, though in the simulations of Feldmann et al. (2011) it arises more generally. The low $\beta$ also agrees qualitatively with numerous observations of highly structured star formation on scales $\lesssim 1 \mathrm{kpc}$ in nearby galaxies; for example, see the recent synthesis by Elmegreen (2011).

From the perspective of galactic-scale star formation, the key point from this calculation is that the scatter in $\tau_{\text {dep }}^{\text {mol }}$ at kpc scales depends on processes operating at scales larger than that of individual star-forming regions. That is, key information on the distribution of star formation in galaxies remains to be extracted from comparison of maps of $\Sigma_{\mathrm{SFR}}$ and $\Sigma_{\text {mol }}$. This represents another manifestation of the overall theme of this section, that

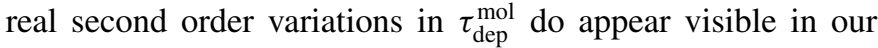
sample. Future investigation of HERACLES and similar surveys will allow tests of the degree to which this correlation can be attributed to systematic variations in $\tau_{\mathrm{dep}}^{\mathrm{mol}}$ as a function of yetunexplored local conditions or to physical parameter estimation. Simultaneously, observations with high spatial dynamic range
Table 8

\begin{tabular}{lc}
\multicolumn{2}{c}{ Scale Dependence of Scatter in $\tau_{\mathrm{dep}}^{\mathrm{mol}}$} \\
\hline Galaxy & Averaging Index, $\beta$ \\
\hline NGC 0628 & $0.5(0.2-0.6)$ \\
NGC 2403 & $0.0(0.0-0.3)$ \\
NGC 2903 & $0.6(0.4-0.6)$ \\
NGC 3627 & $0.5(0.4-0.6)$ \\
NGC 4725 & $0.3(0.3-0.4)$ \\
NGC 4736 & $0.8(0.1-0.8)$ \\
NGC 5194 & $0.6(0.5-0.6)$ \\
NGC 5457 & $0.5(0.1-0.5)$ \\
NGC 6946 & $0.3(0.2-0.4)$ \\
\hline
\end{tabular}

Notes. Averaging index, $\beta$, for well-resolved galaxies. We quote the best-fit $\beta$, defined in Equation (11), estimated from a $\chi^{2}$ minimization using $\mathrm{H} \alpha+24 \mu \mathrm{m}$ and our variable " $\Sigma=100 " \alpha_{\mathrm{CO}}$. In parentheses, we give the range of values measured as we vary SFR tracer and change adopted $\alpha_{\mathrm{CO}}$. For comparison, we expect $\beta \approx 1$ for the case of uncorrelated averaging in a thin disk.

will allow a more detailed diagnosis of the scale-dependence of $\tau_{\mathrm{dep}}^{\mathrm{mol}}$.

\subsection{Enhanced Efficiency in Galaxy Centers}

Our sample does not contain any true galaxy-wide starbursts, but many of our targets do host nuclear concentrations of star formation and gas (Helfer et al. 2003), so that the inner kpc of our targets represents a subsample intermediate between normal disk galaxies and starbursts. These regions represent only a small fraction of the area in our targets, so they exert a negligible impact on the ensemble of data seen in Figure 1 but probe an important part of parameter space-high $\Sigma_{\text {mol }}$, high $\Sigma_{\mathrm{SFR}}$ - and so we explicitly consider them in Figure 13 . We plot the enhancement in $\tau_{\mathrm{dep}}^{\mathrm{mol}}$, calculated by dividing $\tau_{\mathrm{dep}}^{\mathrm{mol}}$ for each point by the average for that galaxy, and mark points from the inner kpc of our targets with large symbols: blue dots for galaxies with some spectroscopic indication of an active galactic nucleus (AGN; Moustakas et al. 2010, or NED), and green stars show star-formation-dominated regions.

The top left panel of Figure 13 shows enhancement in $\tau_{\mathrm{dep}}^{\mathrm{mol}}$ for a fixed $\alpha_{\mathrm{CO}}$. We find systematically lower $\tau_{\mathrm{dep}}^{\text {mol }}$ in the centers of our targets, both AGN and starbursts. We find this shorter $\tau_{\text {dep }}^{\text {mol }}$ using a fixed $\alpha_{\mathrm{CO}}$. Sandstrom et al. (2012) find evidence for systematically lower conversion factors in the central parts of our sample. The top right panel of Figure 13 shows the results of applying these central corrections, as part of our " $\Sigma=100 "$ conversion factor. $\tau_{\mathrm{dep}}^{\mathrm{mol}}$ become even shorter in the central regions compared to the disks. Figure 13 thus exhibits one of the clearest systematic effects in our sample: the central regions of our targets tend to have significantly shorter $\tau_{\mathrm{dep}}^{\mathrm{mol}}$ than the ensemble of disk regions. Central $\tau_{\mathrm{dep}}^{\mathrm{mol}}$ are 0.8 times the disk value with \pm 0.2 dex ( $1 \sigma$ scatter $)$ for fixed $\alpha_{\mathrm{CO}}$. The median central-to-disk ratio drops to 0.6 with \pm 0.35 dex scatter in our $" \Sigma=100 "$ case.

This apparently real shortening of $\tau_{\mathrm{dep}}^{\mathrm{mol}}$ coincides with an increase in the $\mathrm{CO}(2-1)$ to $\mathrm{CO}(1-0)$ ratio, indicative of more excited gas. The bottom right panel shows that this lower $\tau_{\text {dep }}^{\text {mol }}$ coincides with higher CO (2-1) to CO (1-0) ratios (calculated with comparison to Kuno et al. 2007). The central 

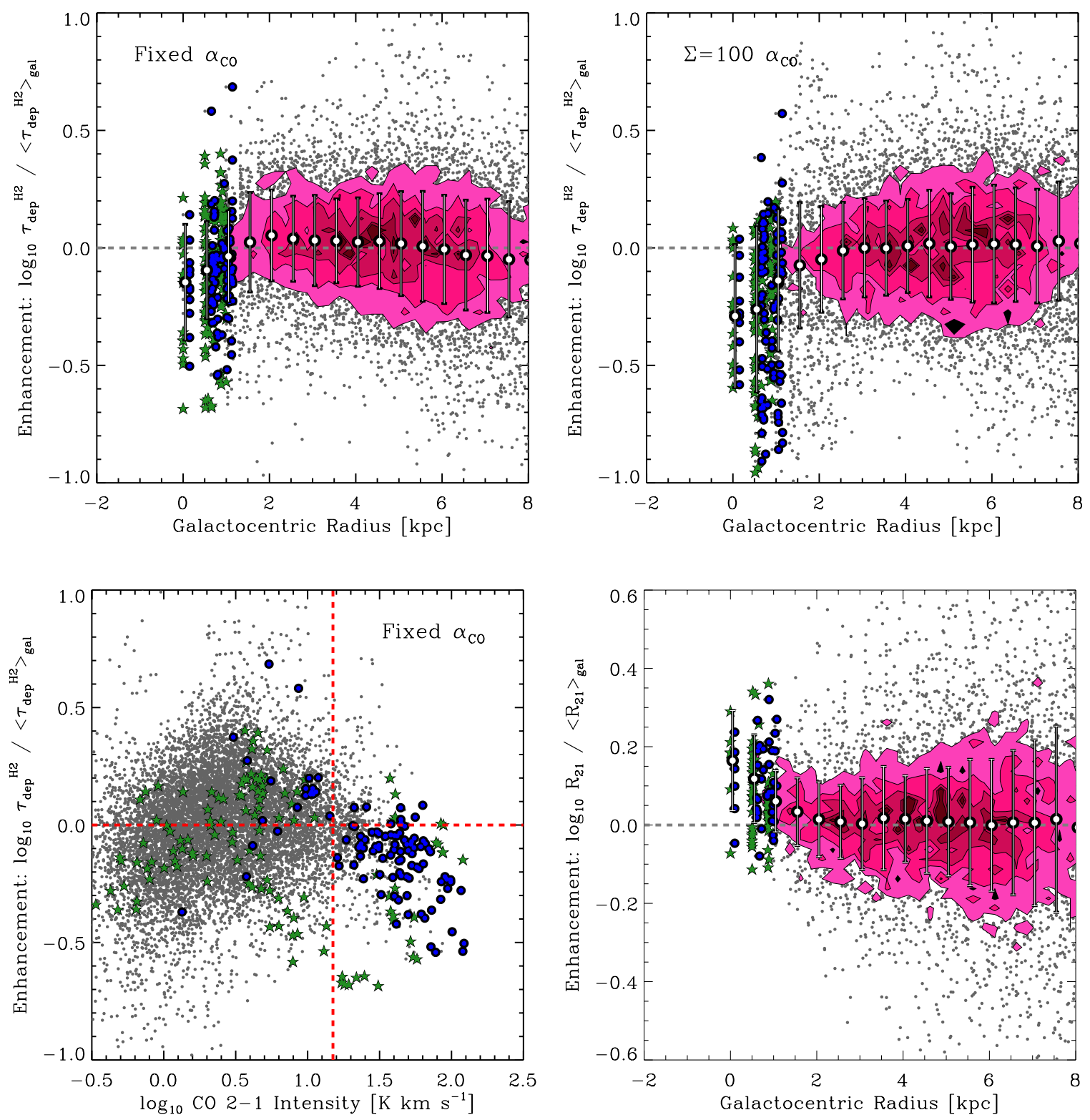

Figure 13. Enhanced efficiency in galaxy centers. We plot $\tau_{\mathrm{dep}}^{\text {mol }}$ normalized to the galaxy average as a function of galactocentric radius for a fixed $\alpha_{\mathrm{CO}}$ (top left) and a " $\Sigma=100$ " conversion factor (top right). We also plot enhancement in $\tau_{\mathrm{dep}}^{\mathrm{mol}}$ as a function of CO (2-1) intensity (bottom left) and show the enhancement in the CO (2-1) to CO (1-0) line ratio relative to the disk average as a function of radius. Individual gray points show kpc-resolution lines of sight; contours show data density; black-and-white points and error bars show median and rms scatter in binned data. Blue points and green stars show data from the inner kpc of our targets-blue points show systems with a spectroscopic classification indicating the likely presence of an AGN (Moustakas et al. 2010); green points show star-formation-dominated nuclei. Galaxy centers show shorter depletion times and enhanced line ratios relative to galaxy disks, indicative of more excited, more efficiently star-forming gas. The effect appears even stronger once we account for variations in $\alpha_{\mathrm{CO}}$ (Sandstorm et al.) and essentially all CO-bright nuclear regions show some level of enhancement.

regions of our targets show systematically enhanced $\mathrm{CO}(2-1)$ / CO (1-0) compared to the disks. This excitement presumably reflects the same changing physical conditions that drive the lower conversion factors found by Sandstrom et al. (2012), underscoring that nuclear gas concentrations represent a distinct physical regime from galaxy disks. Molecular gas in these regions gives off more $\mathrm{CO}$ emission, appears more excited, and forms stars more rapidly than molecular gas further out in the disks of galaxies.

Not all nuclear regions exhibit shorter $\tau_{\text {dep }}^{\mathrm{mol}}$. A sufficient but not necessary condition to find such enhancements in our data is (1) to lie within the central kpc of a target and (2) have $\mathrm{CO}(2-1)$ intensity $\gtrsim 15 \mathrm{~K} \mathrm{kms}^{-1}$. We indicate these criteria using a red line in the bottom left panel of Figure 13. This does not precisely equate to a $\Sigma_{\text {mol }}$ threshold because of ambiguities introduced by $\alpha_{\mathrm{CO}}$; the figure shows that central parts of galaxies with bright $\mathrm{CO}$ emission tend to show lower $\tau_{\mathrm{dep}}^{\mathrm{mol}}$.

Daddi et al. (2010) and Genzel et al. (2010) argue for multiple "modes" or "sequences" of star formation, in which the shorter dynamical time and higher density in starburst galaxies lead to shorter depletion times at fixed $\Sigma_{\text {mol }}$. Figure 13 supports this idea inasmuch as it shows that physical conditions other than gas surface density play an important role determining $\tau_{\text {dep }}^{\text {mol }}$. The population of low $\tau_{\text {dep }}^{\text {mol }}$ points in Figure 13 appear to be driven by factors other than kpc-scale $\Sigma_{\mathrm{mol}}$ alone: first, identifying these lines of sight requires knowing that the points lie in the centers of our targets; second, $\tau_{\mathrm{dep}}^{\mathrm{mol}}$ exhibits a wide range, presumably 
set by other physical parameters. Figure 13 does not support the idea of two cleanly distinguished sequences, suggesting instead that a continuum of $\tau_{\mathrm{dep}}^{\mathrm{mol}}$ exists in the central regions of our targets; we plot the histogram of central $\tau_{\text {dep }}^{\text {mol }}$ in Section 5 .

What drives these enhancements? Beam dilution certainly plays some role. Our kpc resolution will average out nuclear gas concentrations with surface densities well in excess of the disks of our targets but small spatial extent (e.g., Jogee et al. 2005). We expect that high pressure, driven by the deep potential well in the central parts of galaxies, also plays a role driving gas to higher densities. This effect is seen in our own Galaxy (Oka et al. 2001) and others (Rosolowsky \& Blitz 2005). Daddi et al. (2010) and Genzel et al. (2010) suggest that the shorter dynamical timescales in starburst galaxies may also play a key role, and our data may offer some tentative support for this (Figure 10).

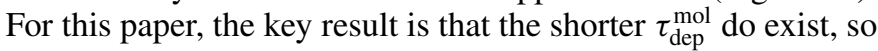
that the inner parts of disk galaxies represent a kind of "transition regime" between the disks of spirals and galaxy-wide starbursts. Follow-up interferometry and spectroscopy contrasting physical conditions in the nuclear regions with those in disks will yield more insight.

\section{DISCUSSION AND CONCLUSIONS}

We study the relationship between molecular gas surface density, $\Sigma_{\text {mol }}$, as traced by CO (2-1) emission and SFR surface density, $\Sigma_{\mathrm{SFR}}$ - traced by combinations of $\mathrm{H} \alpha, \mathrm{FUV}$, and IR emission-at $1 \mathrm{kpc}$ resolution across the optical disks of 30 nearby spiral galaxies. Broadly, we demonstrate two conclusions, which we illustrate using histograms of $\tau_{\text {dep }}^{\text {mol }}$ in Figure 14: a first-order simple correspondence between $\Sigma_{\text {mol }}$ and $\Sigma_{\mathrm{SFR}}$ and second-order systematic variations in the apparent molecular gas depletion time, $\tau_{\mathrm{dep}}^{\mathrm{mol}}$, including lower values in nuclear starbursts and low-mass, low-metallicity galaxies. Some, but not all, of these variations may be explained by invoking a dependence of the CO-to- $\mathrm{H}_{2}$ conversion factor, $\alpha_{\mathrm{CO}}$, on dust-to-gas ratio.

First, molecular gas and star formation are tightly correlated in both individual galaxies and over individual kpc-resolution lines of sight. Their ratio, the molecular gas depletion time, $\tau_{\mathrm{dep}}^{\mathrm{mol}}$, appears to first order constant across the disks of massive, large star-forming galaxies, with a median value $\tau_{\text {dep }}^{\text {mol }}=2.2 \mathrm{Gyr}$, a scatter of $\approx 0.3 \mathrm{dex}$, and systematic uncertainty $\sim 60 \%$ (see the nearly log-normal histogram in the left panels of Figure 14). We arrive at this conclusion testing a wide range of methodologies to trace the recent SFR and distribution of molecular gas. This includes the interchange of $\mathrm{H} \alpha$ and FUV emission, varying treatments of the infrared "cirrus," substitution of literature CO (1-0) for our CO (2-1) maps, and adoption of variable CO-to$\mathrm{H}_{2}$ conversion factors. We demonstrate that our measurements agree with a large collection of literature data from the last decade (Section 3.4), with all data occupying a common region of $\Sigma_{\text {mol }}-\Sigma_{\text {SFR }}$ parameter space. Our data reinforce and extend a consensus for a "large disk galaxy" value of $\tau_{\text {dep }}^{\text {mol }} \approx 2$ Gyr for matched assumptions about the $\mathrm{CO}$-to- $\mathrm{H}_{2}$ conversion factor and stellar IMF with $\approx 0.3$ dex scatter.

Adopting a forward-modeling approach similar to that of Blanc et al. (2009), we derive a best-fit power-law index of $N \approx 1 \pm 0.2$ for $\Sigma_{\mathrm{SFR}} \propto \Sigma_{\text {mol }}^{N}$ (Section 3.3) for a fixed $\alpha_{\mathrm{CO}}$ and show that individual galaxies exhibit a distribution of $N$ with $N$ mostly in the range $0.8-1.2$. However, we stress the inadequacy of a power-law to capture important changes in physical conditions other than $\Sigma_{\text {mol }}$ and the substantial uncertainty in such fits. Reinforcing the conclusions of Blanc et al. (2009), we caution that the commonly used combination of "sigma-clipping" and bivariate fitting has the potential to substantially bias results (see Appendix C), yielding seemingly discordant values of $N$ even when the data substantially agree.

Our second major conclusion is that with a broad sample spanning a wide range of physical conditions, systematic variations in the apparent $\tau_{\mathrm{dep}}^{\mathrm{mol}}$ emerge both among and within galaxies (see the width of the distribution in the top middle panel of Figure 14). We show systematic variations of galaxy-average apparent $\tau_{\text {dep }}^{\text {mol }}$ as a function of many host galaxy properties: stellar mass, rotation velocity, metallicity, dust-to-gas ratio, average gas surface density, and morphology. These variations have the sense that low-mass, low-metallicity, late-type galaxies exhibit shorter apparent $\tau_{\text {dep }}^{\text {mol }}$ than high-mass galaxies. The trends persist, though weaker, even into the high-mass region, $M_{*}>10^{10} M_{\odot}$, and agree well with those seen in the COLDGASS sample (Saintonge et al. 2012). We emphasize "apparent" because these variations appear to be a mixture of real changes in the rate at which gas forms stars and biases in physical parameter estimation. Adopting a CO-to- $\mathrm{H}_{2}$ conversion factor that depends on the dust-to-gas ratio can explain many of the strongest variations with host galaxy properties; note the narrowing from the top middle panel of Figure 14 to the bottom middle panel. Our ability to examine residual trends remains restricted by the limited precision with which we know $\alpha_{\mathrm{CO}}$, but our best estimate is that correlations do remain between the real ( $\alpha_{\mathrm{CO}}$-adjusted) $\tau_{\mathrm{dep}}^{\mathrm{mol}}$ and galaxy mass, average gas surface density, and perhaps several other quantities.

We also examine how $\tau_{\text {dep }}^{\text {mol }}$ varies as a function of local conditions. We find two strong relationships: the apparent $\tau_{\mathrm{dep}}^{\mathrm{mol}}$ calculated for a fixed $\alpha_{\mathrm{CO}}$ varies systematically as a function of dust-to-gas ratio, and we observe systematically lower and widely varying $\tau_{\mathrm{dep}}^{\mathrm{mol}}$ in the inner kpc of our targets (see the top right panel of Figure 14). We interpret the first as indicating important variations in $\alpha_{\mathrm{CO}}$ and, as with the galaxy-integrated case, show that application of a dust-to-gas ratio-dependent $\alpha_{\mathrm{CO}}$ can explain much of the observed trend. The lower $\tau_{\text {dep }}^{\text {mol }}$ in galaxy centers appears real and robust to $\alpha_{\mathrm{CO}}$ considerations. Indeed, Sandstrom et al. (2012) find low $\alpha_{\mathrm{CO}}$ in the central parts of many of our targets, which implies even lower $\tau_{\mathrm{dep}}^{\mathrm{mol}}$. The resulting $\tau_{\mathrm{dep}}^{\mathrm{mol}}$ in the inner parts of galaxies varies widely (see the right panels in Figure 14), providing strong evidence that environmental factors do drive $\tau_{\mathrm{dep}}^{\mathrm{mol}}$, and thus the SFR, in these regions.

Strong local trends beyond those linking $\tau_{\mathrm{dep}}^{\mathrm{mol}}$ to the dustto-gas ratio and nuclear starbursts elude our present analysis,

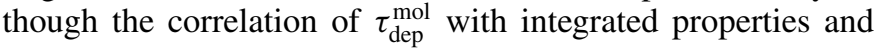
weak dependence of scatter on scale strongly suggest their presence. We suspect that the limited resolution and remaining imprecision in physical parameter estimation so far obscure these trends in HERACLES. Comparison to existing Herschel data and spectroscopy of other molecular lines should improve our ability to understand the origins of the physical scatter in $\tau_{\text {dep }}^{\text {mol }}$. Perhaps just as important, at kpc resolution significant averaging has already occurred, especially in regions of high $\Sigma_{\text {SFR }}$. The star-forming ISM hosts many competing effects: for example, shear may both suppress collapse and lead to more frequent cloud collisions; high pressures may lead to both denser clouds and a substantial diffuse molecular ISM; spiral arms may both collect material and suppress collapse via streaming motions. At our kpc resolution, such trends will be subtle as 


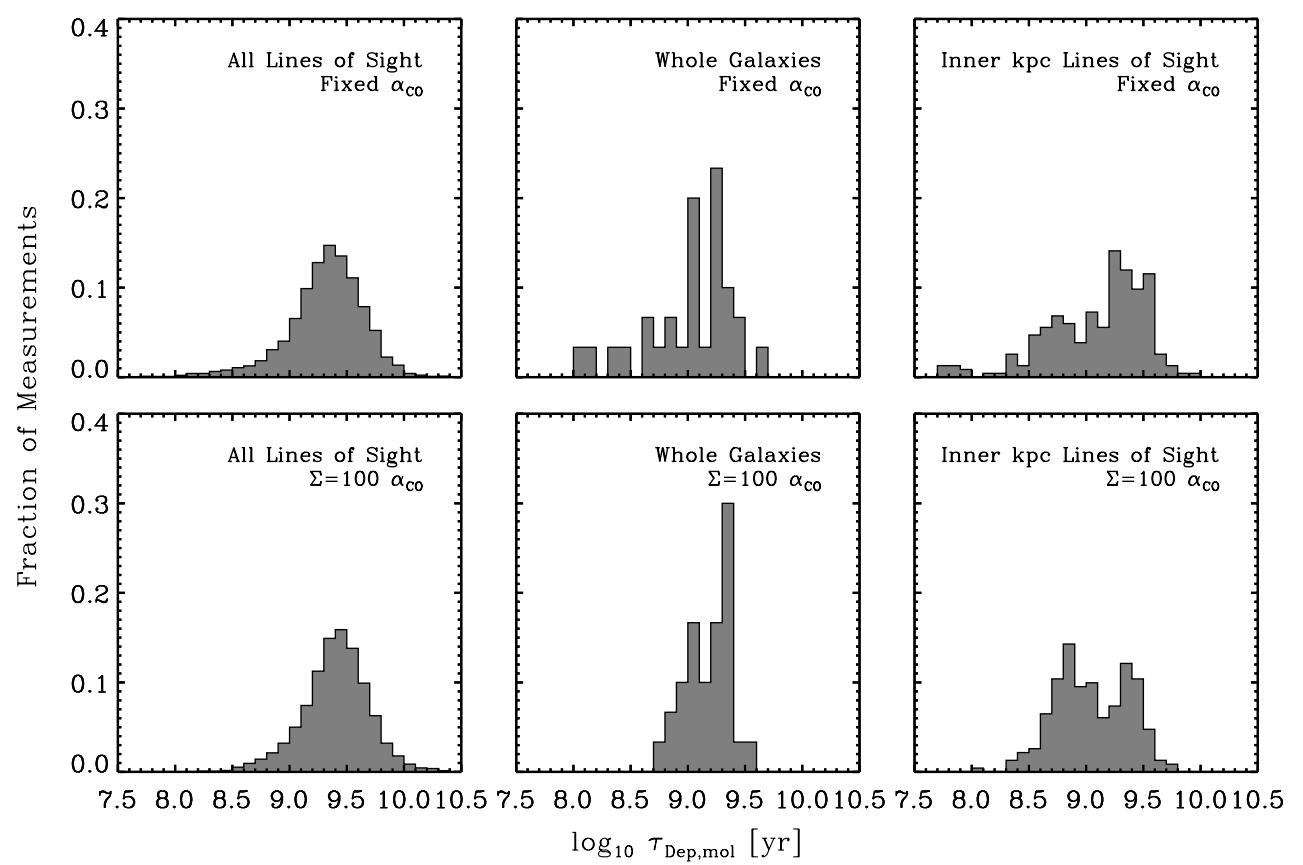

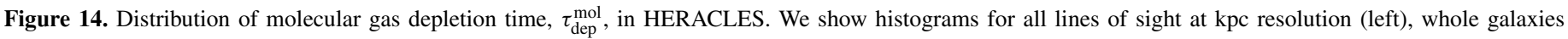

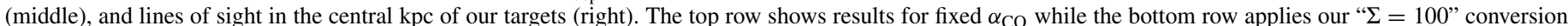

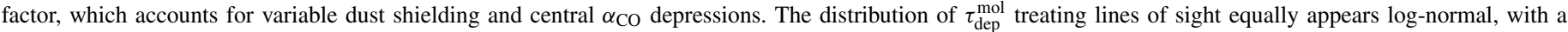

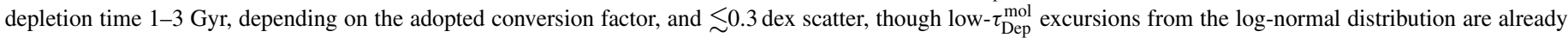

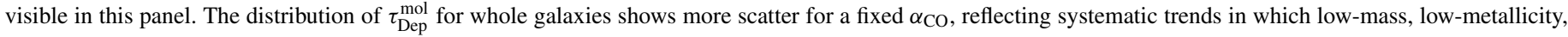

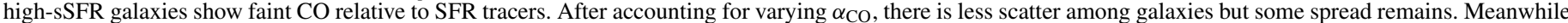

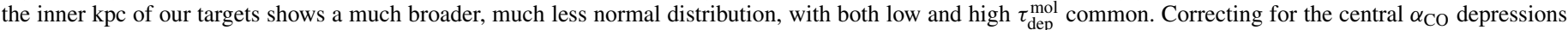

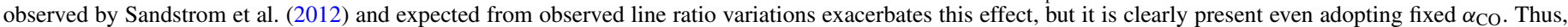
$\tau_{\mathrm{dep}}^{\mathrm{mol}}$ appears constant to first order, but clear systematic second-order variations do emerge as a function of local and galaxy scale conditions in a broad sample.

competing effects occur inside a single resolution element. In the longer term, higher resolution observations of a diverse sample of galaxies will be needed to diagnose the impact of dynamical effects on star formation.

These results fit into a broad picture of star formation in galaxies as follows. Within the disks of nearby galaxies, we find recent star formation correlated with molecular, rather than atomic or total, gas (Schruba et al. 2011). The ratio of CO emission to recent star formation appears roughly constant within massive, star-forming disk galaxies (Section 3; Bigiel et al. 2011), but when examined more closely, significant variations do emerge between this ratio and galaxy mass (Saintonge et al. 2012; Section 4) and dust-to-gas ratio or metallicity (Section 4). The interpretation of these trends depends critically on the behavior of the $\mathrm{CO}-$ to- $\mathrm{H}_{2}$ conversion factor. We show that current best estimates may explain many of the observed trends in the CO-to-SFR ratio (see also Bolatto et al. 2011; Schruba et al. 2012). However, we caution that significant work is still needed to bring conversion factor estimates to the precision needed to confidently interpret these trends. Our best estimate is that correlations in which the true molecular gas depletion time, $\tau_{\mathrm{dep}}^{\mathrm{mol}}$, increases with increasing galaxy mass or gas surface density do persist after accounting for $\alpha_{\mathrm{CO}}$ effects, but that these are comparatively weak. A sensible explanation for such trends is the emergence of a diffuse, unbound molecular medium at high gas surface densities and high pressures, but direct evidence relating a local high molecular fraction to lower $\tau_{\text {dep }}^{\text {mol }}$ is weak in our present data set. We do find good evidence for systematically low $\tau_{\mathrm{dep}}^{\mathrm{mol}}$ in galaxy centers, with a wide variation in the factor

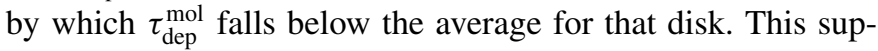

ports the idea that in environments with high surface densities and short dynamical or orbital times, environmental factors may drive $\tau_{\mathrm{dep}}^{\mathrm{mol}}$ to a wide range of values at fixed average gas surface density. This is a more general formulation than the idea of distinct "disk" and "starburst" sequences, but qualitatively agrees with the picture from Daddi et al. (2010) and Genzel et al. (2010), with the nuclei of disk galaxies occupying a regime intermediate between quiescent disks and merger-induced starbursts.

We thank the referee for a constructive report that led to significant improvements in this paper. We also thank Robert Feldmann, Scott Schnee, Guillermo Blanc, Harvey Liszt, and David Whelan for useful discussions and feedback. We thank the GALEX NGS, SINGS, LVL, and COLDGASS teams for making their outstanding data sets available. We thank staff of the IRAM $30 \mathrm{~m}$ for their assistance carrying out the HERACLES survey. We thank Deidre Hunter for sharing her H $\alpha$ image of NGC 4214. F.B., A.K.L., and F.W. gratefully acknowledge the Aspen Center for Physics, where part of this work was carried out. Support for A.K.L. for part of this project was provided by NASA through Hubble Fellowship grant HST-HF-51258.01-A awarded by the Space Telescope Science Institute, which is operated by the Association of Universities for Research in Astronomy, Inc., for NASA, under contract NAS 5-26555. K.S. is supported by a Marie Curie International Incoming Fellowship. J.C.M.M. acknowledges financial support from NASA JPL/Spitzer grant RSA 1374189 provided for the S4G project. A.B. wishes to acknowledge partial support from grants NSF AST-0838178, NSF AST-0955836, as well as a Cottrell Scholar award from the 

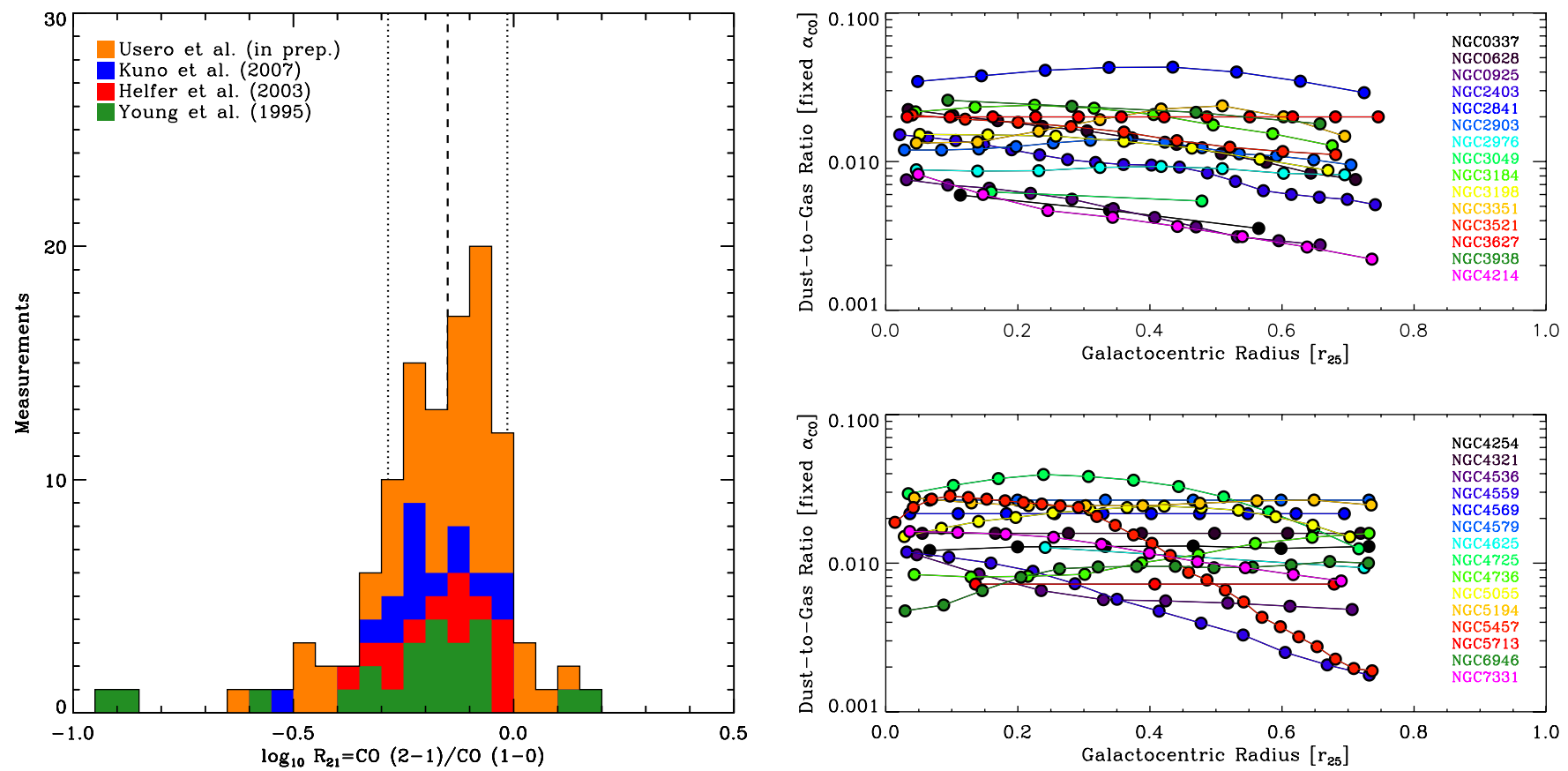

Figure 15. Line ratios and dust-to-gas ratios in HERACLES. Left: $\mathrm{CO}(2-1) / \mathrm{CO}(1-0)$ line ratio comparing HERACLES integrated measurements to literature CO (1-0) surveys: Kuno et al. (2007), Helfer et al. (2003), Young et al. (1995), and measurements for individual pointings obtained for follow-up spectroscopy by A. Usero et al. (in preparation). The ensemble of measurements has median 0.67 with scatter 0.16 dex. Right: dust-to-gas ratio profiles derived comparing IR SED modeling, HERACLES, and $\mathrm{H}$ I data. We plot the radial profile of the dust-to-gas ratio derived using a fixed conversion factor.

Research Corporation for Science Advancement. We have made use of the NASA/IPAC Extragalactic Database (NED), which is operated by the Jet Propulsion Laboratory, California Institute of Technology, under contract with the National Aeronautics and Space Administration. We also acknowledge use of the Lyon Extragalactic Database (LEDA) and NASA's Astrophysics Data System (ADS). The National Radio Astronomy Observatory is a facility of the National Science Foundation operated under cooperative agreement by Associated Universities, Inc.

\section{APPENDIX A}

\section{MEAN LINE RATIO FOR HERACLES}

HERACLES surveyed the CO (2-1) transition. Our adopted "fixed" $\alpha_{\mathrm{CO}}=4.35$ has been derived considering mainly CO (1-0) observations, and most of the literature comparing recent star formation and molecular gas considers CO (1-0). E. Rosolowsky et al. (in preparation) present a thorough analysis of the line ratio as a function of local conditions in HERACLES and explore the implications for $\alpha_{\mathrm{CO}}$ and $\tau_{\mathrm{dep}}^{\mathrm{mol}}$. In this study we adopt a fiducial $\mathrm{CO}(2-1) / \mathrm{CO}(1-0)$ ratio of 0.7 . The left panel in Figure 15 shows a histogram of $\mathrm{CO}(2-1) / \mathrm{CO}(1-0)$ values measured for the HERACLES survey. We plot ratios derived from comparing integrated HERACLES fluxes to those from the CO (1-0) surveys by Young et al. (1995), Helfer et al. (2003), and Kuno et al. (2007). We also show the results of pointed single-pixel spectroscopy carried out with the IRAM $30 \mathrm{~m}$, part of a large spectroscopic database presented by A. Usero et al. (in preparation). These have been reduced using CLASS in a standard way and aperture-corrected to match beam areas using the $\mathrm{CO}(2-1)$ distribution in HERACLES. The ensemble of measurements has median 0.67 , with the ratio taken in brightness temperature units, and a scatter of 0.16 dex, $\approx 40 \%$. Some of this scatter will be due to calibration uncertainties in the CO (1-0) data. However, even comparing each data set to
HERACLES separately, we find internal scatter in CO (2-1)/CO (1-0) of $20 \%-40 \%$. We discuss the most obvious environmental dependence of this ratio, the enhancement in galaxy centers in Section 4.4 (see also Leroy et al. 2009). Based on Figure 15, we adopt 0.7 as a typical line ratio. In this paper, the primary application of this value is to apply a "standard" Milky Way CO (1-0) conversion factor to the HERACLES CO (2-1) data.

\section{APPENDIX B}

\section{DUST-TO-GAS RATIO AND CONVERSION FACTOR CALCULATIONS}

The right panel in Figure 15 plots the dust-to-gas ratios, $\mathrm{D} / \mathrm{G}$, that we derive from a fixed $\alpha_{\mathrm{CO}}$ conversion factor and our Spitzer SED modeling.

We use the recent theoretical work by Wolfire et al. (2010) to estimate the fraction of "CO-dark" molecular gas. This gas lies in regions where carbon is mostly associated with $\mathrm{C}$ II rather than $\mathrm{CO}$, and so will not be readily traced by maps of $\mathrm{CO}$ emission (see Krumholz et al. 2011, for a similar approach). Wolfire et al. (2010) present an expression for the fraction of mass in this "CO-dark" phase,

$$
f_{\mathrm{CO}-\text { dark }}=1-\exp \left(\frac{-4.0 \Delta A_{V, D G}}{\overline{A_{V}}\left(\mathrm{D} / \mathrm{G}^{\prime}\right)}\right)
$$

where $\Delta A_{V, D G}$ is the depth that the CO-dark phase extends into the cloud, measured in units of visual extinction, and $\overline{A_{V}}$ is mean extinction through the whole cloud. $\overline{A_{V}}$ simply depends on the product of cloud surface density-or equivalently mean extinction through the cloud at solar metallicity, $\overline{A_{V}^{0}}$ - and the dust-to-gas ratio, $\overline{A_{V}}\left(\mathrm{D} / \mathrm{G}^{\prime}\right)=\overline{A_{V}^{0}} \mathrm{D} / \mathrm{G}^{\prime}$. Wolfire et al. (2010) give an expression for $\Delta A_{V, D G}$ that depends weakly on the 

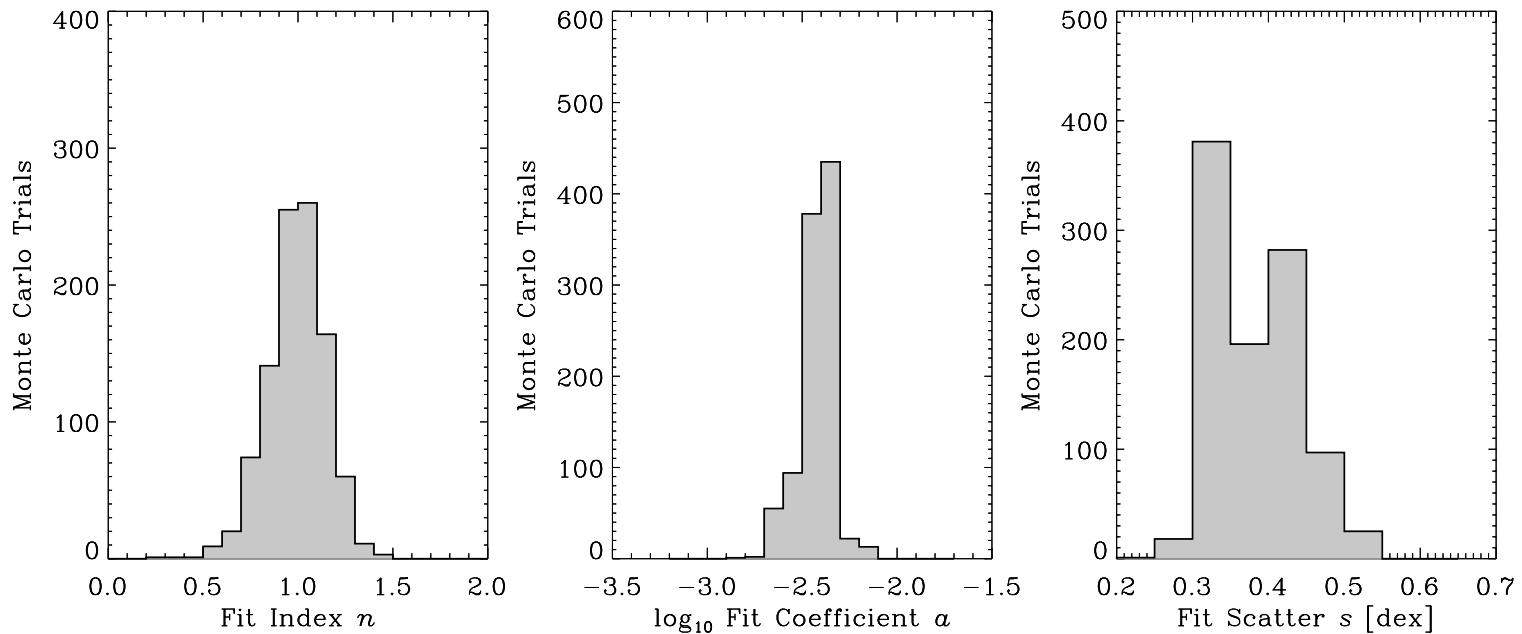

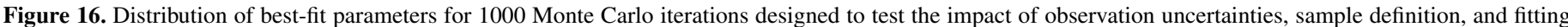

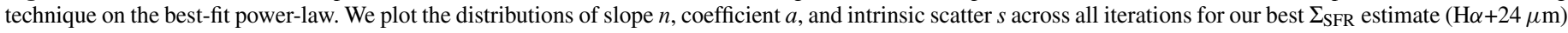
and a fixed $\alpha_{\mathrm{CO}}$.

density and metallicity of the cloud

$$
\Delta A_{V, D G}=0.53-0.045 \ln \left(\frac{G_{0}^{\prime}}{n_{c}}\right)-0.097 \ln Z^{\prime},
$$

where $G_{0}^{\prime}$ is the radiation field relative to the solar neighborhood value, $n_{c}$ is the density of the cloud, and $Z^{\prime}$ is the metallicity (or dust-to-gas ratio) relative to the Galactic value. We neglect the second term, setting $G_{0}^{\prime}=1$ and $n_{c}=1 \mathrm{~cm}^{-3}$, and use the $\mathrm{D} / \mathrm{G}^{\prime}$ in place of $Z^{\prime} . \Delta A_{V, D G}$ does not vary much with any of these quantities, so that $f_{\mathrm{CO}-\text { dark }}$ mainly depends on the extinction through the cloud, $\bar{A}_{V}\left(\mathrm{D} / \mathrm{G}^{\prime}\right)$.

We scale the conversion factor by this expression, so that

$$
\begin{gathered}
\alpha_{\mathrm{CO}} \propto \frac{1}{1-f_{\mathrm{CO}-\text { dark }}} \\
\alpha_{\mathrm{CO}} \propto\left(\exp \left(\frac{-4.0 \Delta A_{V, D G}}{\overline{A_{V}}(\mathrm{D} / \mathrm{G})}\right)\right)^{-1} \\
\alpha_{\mathrm{CO}} \propto \exp \left(\frac{4.0 \Delta A_{V, D G}}{\overline{A_{V}}(\mathrm{D} / \mathrm{G})}\right) .
\end{gathered}
$$

Adopting the conversion between dust and column density of Bohlin et al. (1978) and a fiducial average molecular cloud surface density of $100 M_{\odot} \mathrm{pc}^{-2}$, so that $A_{V} \approx 4.9$, we calculate the correction for "CO-dark" gas via

$$
\begin{aligned}
c_{\mathrm{CO}-\text { dark }}\left(\mathrm{D} / \mathrm{G}^{\prime}\right) & =\alpha_{\mathrm{CO}}\left(\mathrm{D} / \mathrm{G}^{\prime}\right) / \alpha_{\mathrm{CO}}\left(\mathrm{D} / \mathrm{G}^{\prime}=1\right) \\
& =\alpha_{\mathrm{CO}}\left(\mathrm{D} / \mathrm{G}^{\prime}\right) / \alpha_{\mathrm{CO}}\left(\mathrm{D} / \mathrm{G}^{\prime}=1\right) \\
& =\exp \left(\frac{4.0 \times 0.53}{4.9 \Sigma_{100} \mathrm{D} / \mathrm{G}^{\prime}}\right) / \exp \left(\frac{4.0 \times 0.53}{4.9}\right) \\
& =0.65 \exp \left(\frac{0.4}{\Sigma_{100} \mathrm{D} / \mathrm{G}^{\prime}}\right)
\end{aligned}
$$

where $\Sigma_{100}$ is the assumed universal surface density of molecular clouds normalized to $100 M_{\odot} \mathrm{pc}^{-2}$ and $\mathrm{D} / \mathrm{G}^{\prime}$ is the dust-to-gas ratio normalized to the Milky Way value. This is Equation (5).

\section{APPENDIX C}

\section{ISSUES IN POWER-LAW FITTING}

\section{C.1. Monte Carlo Uncertainty Estimates}

If we treat our goodness-of-fit statistic (Equation (10)) as $\chi^{2}$, then the implied statistical uncertainties on our fits are very small (the $\chi^{2}+1$ surface implies uncertainties of order $1 \%$, as in Blanc et al. 2009). At some level, this is accurate: given our fitting approach and data set, the best-fit power-law is heavily constrained. However, this does not reflect our real best estimate of the uncertainty in the underlying relationship between $\Sigma_{\text {SFR }}$ and $\Sigma_{\text {mol }}$. To estimate a more realistic uncertainty, we carry out a series of Monte Carlo simulations. We begin with our measurements, estimates of $\Sigma_{\text {mol }}$ and $\Sigma_{\text {SFR }}$ at each point. We then examine the effects of the following.

1. Statistical noise. For each point, we add normally distributed noise of the appropriate magnitude to each $\Sigma_{\text {mol }}$ and log-normally distributed noise of magnitude $0.15 \mathrm{dex}$ to $\Sigma_{\mathrm{SFR}}$ (see Section 2).

2. Calibration uncertainties. Calibration issues include uncertainties in the overall flux scale of the data or uncertainties in the conversion to physical parameters, e.g., due to variations in CO line ratios, dust properties, or stellar populations. In each case, these will tend to operate galaxy by galaxy. We take these to be log-normally distributed with a magnitude of 0.15 dex (Leroy et al. 2009, L12). We scale all of the data for each axis in each galaxy by a single, randomly generated factor.

3. Robustness to removal of individual data. We test the robustness of the results to the removal of individual data using a standard bootstrapping approach. We resample the data, allowing repeats, to produce a data set matched in size to the original.

4. Robustness to removal of galaxies. We also test the robustness of our fit to the removal of whole galaxies using a bootstrapping approach. Instead of resampling the ensemble of measurements, we resample the list of galaxies, allowing repeats, to produce a sample of 30 (non-unique) galaxies.

5. Choice of fitting parameters. A subtle point in our fitting is how to handle data in cells with low (or zero) model 

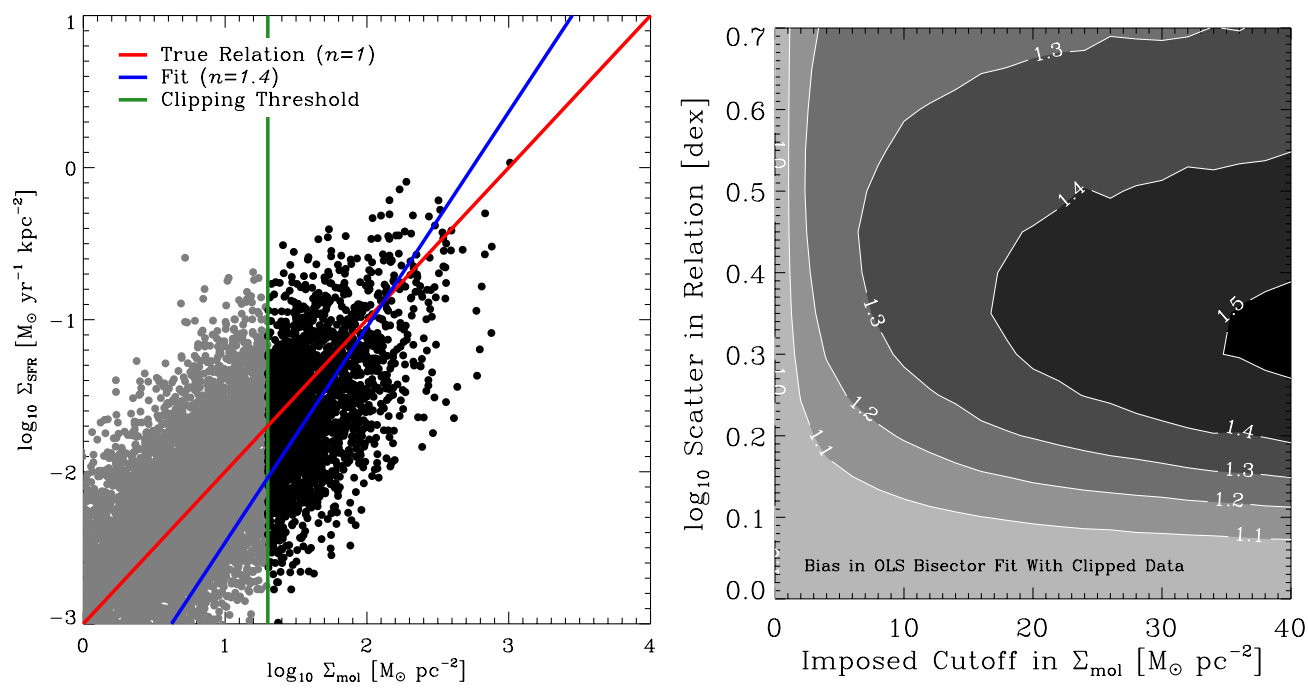

Figure 17. Left: example of the biases introduced by clipping data on one axis (e.g., due to limited $\mathrm{S} / \mathrm{N}$ ) before carrying out a bivariate fit. Points show a simulated data set in which $\Sigma_{\mathrm{SFR}} \propto \Sigma_{\text {mol }}$ by construction (red line) with 0.3 dex scatter introduced to each axis. We clip data below $\Sigma_{\text {mol }}=20 M_{\odot}$ pc ${ }^{-2}$ so that gray points are discarded and black points are fit. We then fit the black points using the OLS bisector method and plot the result in blue. The result is a bias toward higher slope. Right: derived power-law index from using the OLS bisector to fit noisy, low dynamic range data with clipping applied to one axis. The underlying model has an intrinsic $n=1.0$ slope with $\Sigma_{\text {mol }}$ drawn from our data and log-normal scatter of the indicated magnitude applied to each axis. The gray scale and contours report the best-fit power-law index after the data are clipped at the $x$-axis value in one axis and fit using the OLS bisector technique. Biases of several tenths up to as much as four tenths are possible within the range of values adopted by recent studies.

Table 9

Monte Carlo Estimates of Uncertainty Power-law Fit Parameters

\begin{tabular}{lccc}
\hline \hline Source of Uncertainty & Scatter in $n$ & Scatter in $\log _{10} a$ & Scatter in $s$ \\
\hline Statistical noise & 0.05 & 0.01 & 0.02 \\
Calibration uncertainties & 0.12 & 0.06 & 0.04 \\
Robustness to removal of individual data & 0.07 & 0.03 & 0.02 \\
Robustness to removal of galaxies & 0.10 & 0.06 & 0.05 \\
Choice of fitting parameters & 0.06 & 0.02 & 0.02 \\
\hline Overall uncertainty & 0.15 & 0.09 & 0.05 \\
\hline
\end{tabular}

Notes. Each entry gives the $1 \sigma$ standard deviation in the parameter across 100 Monte Carlo iterations (1000 for the overall uncertainty). We quote results for our best-estimate $\Sigma_{\mathrm{SFR}}, \mathrm{H} \alpha+24 \mu \mathrm{m}$ corrected for IR cirrus, and a fixed $\alpha_{\mathrm{CO}}$.

probability. A single outlying datum can dramatically skew the results if this issue is not properly treated. By default, we follow Blanc et al. (2009) and G. Blanc (2012, private communication) and never allow the weight for a single cell to be lower than expected for $N_{\text {model }}^{i}=1$ (i.e., we cap the denominator in Equation (10) at this level). We test the impact of this choice and our adopted grid cell size $(0.125$ dex $)$. We allow the bin size and the minimum weight per cell to vary by up to a factor of two either higher or lower, with equal probability across the range.

We test each of these sources alone and report the results for $\Sigma_{\mathrm{SFR}}$ traced by $\mathrm{H} \alpha$ and $24 \mu \mathrm{m}$ and $\Sigma_{\text {mol }}$ calculated from a fixed $\alpha_{\mathrm{CO}}$ in Table 9. Figure 16 shows the distribution of fitted parameters across all runs for our best $\Sigma_{\text {SFR }}$ estimate. The strongest contributors to the overall uncertainty are galaxy-togalaxy calibration uncertainties or sample definition. Our large data set and good $\mathrm{S} / \mathrm{N}$ make statistical noise over individual lines of sight or removal of individual data minor concerns. Choice of fitting parameters does impact the overall results, but does not dominate the uncertainty.

In the end, we derive our overall uncertainty on the fits from the scatter in the best-fit parameters across 1000 Monte Carlo iterations that include all of these effects. Because we can only add noise or decrease our sample size, each Monte Carlo iteration operates on a data set of inferior quality to that used for our best estimates (Table 4). We consider these uncertainties realistic but conservative.

\section{C.2. Power-law Index Biases in Bivariate Fits to Clipped Data}

Fitting a relationship between $\Sigma_{\text {SFR }}$ and $\Sigma_{\text {mol }}$ entails conducting a bivariate fit to data with limited sensitivity. The approach of Blanc et al. (2009) incorporates upper limits. However, it has been common practice to clip data at some signal-to-noise threshold in $\mathrm{CO}\left(\Sigma_{\mathrm{mol}}\right)$ and then to carry out a bivariate linear fit relating $\log _{10} \Sigma_{\text {SFR }}$ to $\log _{10} \Sigma_{\text {mol }}$ above this threshold, e.g., using the ordinary least squares (OLS) bisector (Isobe et al. 1990) or FITEXY (Press et al. 1992) methods. This clip-and-fit approach can introduce a significant bias into the fit power-law index. We note this effect and its magnitude here.

The bias arises from the interaction of the clipping with fitting techniques that attempt to minimize the two-dimensional distance between a point and the fit line (in $\log -\log$ space). Consider data that are intrinsically defined by a power-law, a line in $\log -\log$ space, but exhibit significant scatter orthogonal to the line. Now consider data near the clipping threshold in $\Sigma_{\text {mol }}$, the quantity that typically represents the limiting observable. Data 


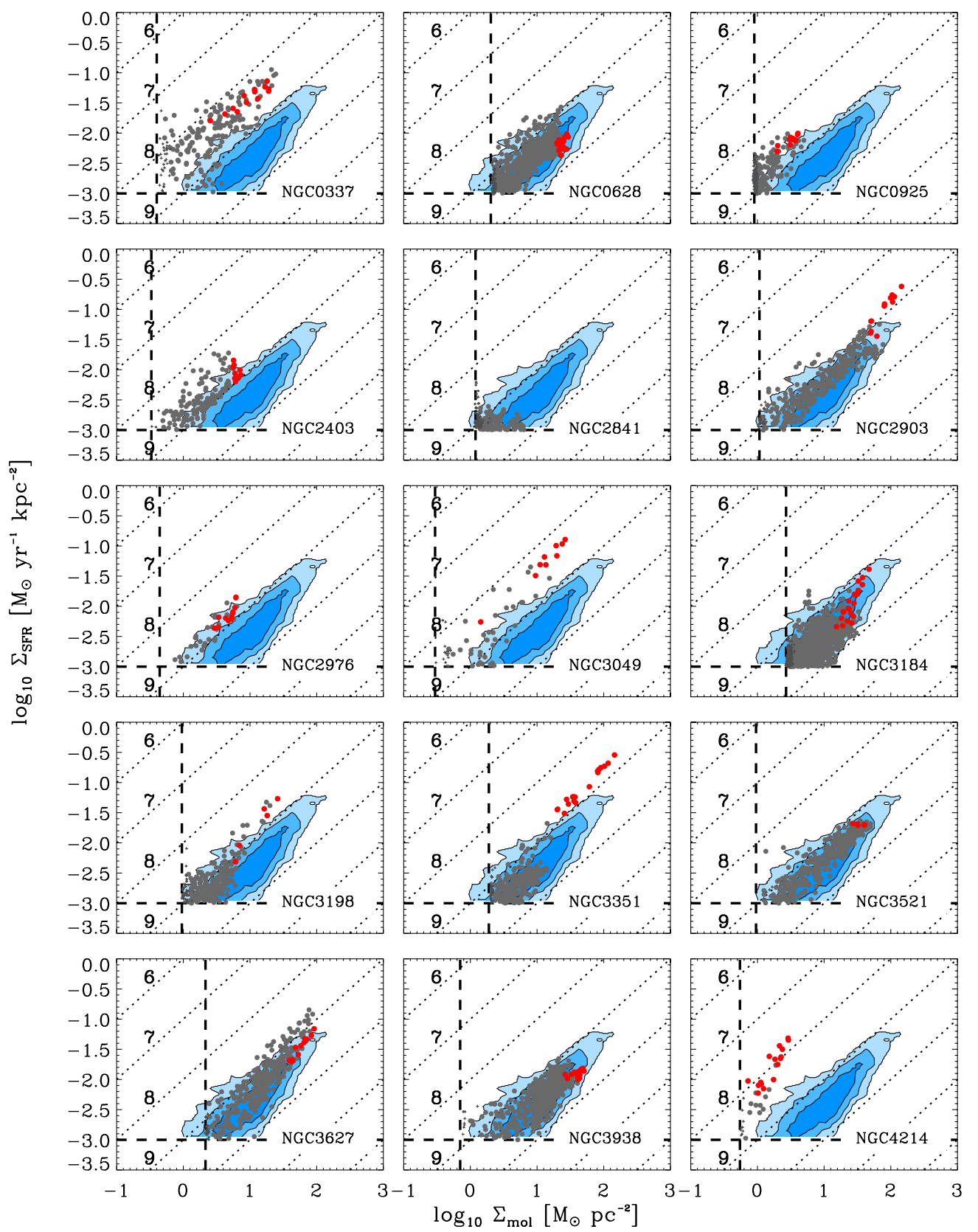

Figure 18. $\Sigma_{\mathrm{SFR}}$, estimated from $\mathrm{H} \alpha+24 \mu \mathrm{m}$, as a function of $\Sigma_{\mathrm{mol}}$, estimated from HERACLES CO (2-1) data and a fixed $\alpha_{\mathrm{CO}}$, for individual galaxies. Data from the designated galaxy appear in gray, with data from the inner $0.1 r_{25}$ marked in red. In the background, we plot data density contours from the ensemble of all measurements (Figure 1). Annotation and symbols are otherwise as Figure 1.

that scatter to high $\Sigma_{\mathrm{SFR}}$ and low $\Sigma_{\text {mol }}$ will be discarded from the analysis due to the clipping. Data that scatter to low $\Sigma_{\mathrm{SFR}}$ and high $\Sigma_{\text {mol }}$ will be included in the analysis. Preferentially adding data at low $\Sigma_{\mathrm{SFR}}$ and high $\Sigma_{\text {mol }}$ will tend to skew the fit toward steeper slopes. The left panel in Figure 17 illustrates the effect for simulated data.

We estimate the magnitude of the effect using a Monte Carlo simulation. We consider samples of 10,000 data points with $\Sigma_{\text {mol }}$ randomly drawn from our data set. We assume an underlying linear relation, $\Sigma_{\mathrm{SFR}} \propto \Sigma_{\mathrm{mol}}$, so that the true $n=1$. We set $\tau_{\mathrm{dep}}^{\mathrm{mol}}=1 \mathrm{Gyr}$, but the choice is arbitrary. We realize 100 samples of 10,000 data points each. For each sample we introduce lognormal scatter of equal magnitude to each axis and then clip the data at a series of $\Sigma_{\text {mol }}$ values from 0 to $40 M_{\odot} \mathrm{pc}^{-2}$. We then carry out an OLS bisector fit to the data and record the best-fit power-law index. The right panel in Figure 17 plots the average fit index across all Monte Carlo iterations as a function of the scatter introduced into the data ( $y$-axis) and the threshold imposed ( $x$-axis). We find that for commonly used thresholds $\left(\Sigma_{\text {mol }} \sim 10-20 M_{\odot} \mathrm{pc}^{-2}\right)$ and typical observed scatters $(\sim 0.3$ dex $)$ a significant bias can be introduced to the best-fit index, often shifting it from its true value of 1.0 (by construction) to $\sim 1.3$ or 1.4 .

Note that Figure 17 illustrates the problem but that the exact magnitude of the bias will depend on the dynamic range, noise, and underlying relationship in the data set studied. Also note that conversely, clipping based on $\Sigma_{\mathrm{SFR}}$ will tend to bias the index in the opposite direction, to lower $n$. Clipping based on both $\Sigma_{\mathrm{SFR}}$ and $\Sigma_{\text {mol }}$ may lead to offsetting biases but does not represent a proper substitute for a rigorous treatment. Finally, we emphasize 


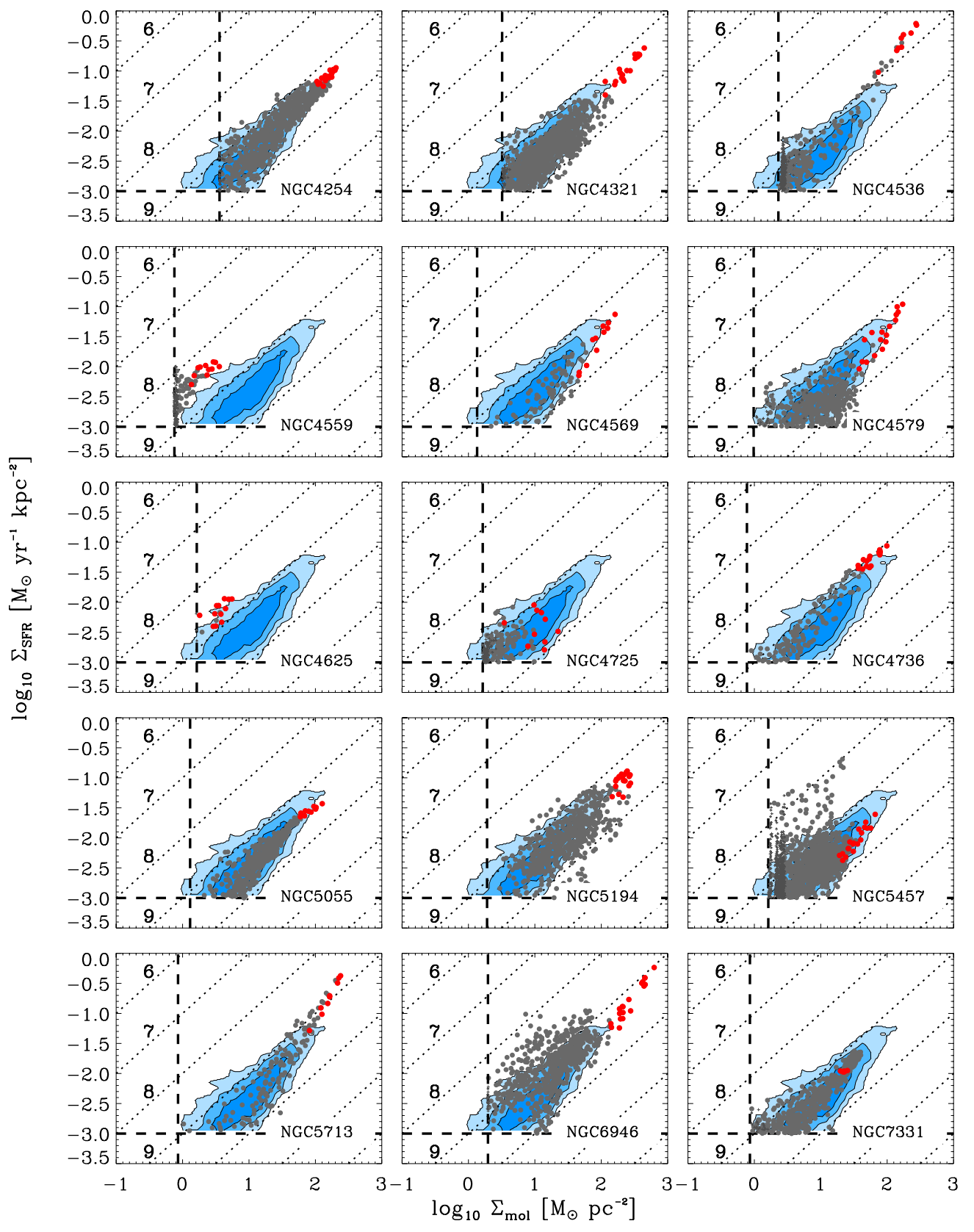

Figure 19. As Figure 18 for the remaining galaxies in our sample.

that although we do clip data below $\Sigma_{\text {mol }}=5 M_{\odot} \mathrm{pc}^{-2}$ in our fitting (Section 3.3), the bias described here will not affect our results because we model the data distribution rather than carry out a bivariate fit.

\section{APPENDIX D}

\section{$\Sigma_{\mathrm{SFR}}-\Sigma_{\text {mol }}$ RELATIONS FOR INDIVIDUAL GALAXIES}

Combining our measurements into a single data set obscures real differences among galaxies. Table 2, Figure 5, and Section 4.1 show systematic variations in average $\tau_{\text {dep }}^{\text {mol }}$ and power-law index among galaxies. In Figures 18 and 19 we plot $\Sigma_{\text {SFR }}$ as a function of $\Sigma_{\text {mol }}$ for individual galaxies. In the background, we plot contours for the combined distribution shown in Figure 1. We show results for our best SFR tracer, $\mathrm{H} \alpha+24 \mu \mathrm{m}$, and a fixed CO-to- $\mathrm{H}_{2}$ conversion factor. Points from the central part of the galaxy, $r_{\text {gal }} \leqslant 0.1 r_{25}$, appear in red. Points from the rest of the galaxy appear in gray.

\section{REFERENCES}

Aniano, G., Draine, B. T., Calzetti, D., et al. 2012, ApJ, 756, 138

Bigiel, F., Leroy, A., Walter, F., et al. 2008, AJ, 136, 2846

Bigiel, F., Leroy, A., Walter, F., et al. 2010, AJ, 140, 1194

Bigiel, F., Leroy, A. K., Walter, F., et al. 2011, ApJL, 730, L13

Blanc, G. A., Heiderman, A., Gebhardt, K., Evans, N. J., \& Adams, J. 2009, ApJ, 704, 842

Blitz, L., \& Rosolowsky, E. 2006, ApJ, 650, 933

Bohlin, R. C., Savage, B. D., \& Drake, J. F. 1978, ApJ, 224, 132

Boissier, S., Prantzos, N., Boselli, A., \& Gavazzi, G. 2003, MNRAS, 346, 1215

Böker, T., Lisenfeld, U., \& Schinnerer, E. 2003, A\&A, 406, 87

Bolatto, A. D., Leroy, A. K., Jameson, K., et al. 2011, ApJ, 741, 12

Bolatto, A. D., Leroy, A. K., Rosolowsky, E., Walter, F., \& Blitz, L. 2008, ApJ, 686, 948

Calzetti, D., Wu, S., Hong, S., et al. 2010, ApJ, 714, 1256

Chen, C., Indebetouw, R., Chu, Y., et al. 2010, ApJ, 721, 1206 
Condon, J. J. 1992, ARA\&A, 30, 575

Crosthwaite, L. P., \& Turner, J. L. 2007, AJ, 134, 1827

Daddi, E., Elbaz, D., Walter, F., et al. 2010, ApJL, 714, L118

Dalcanton, J. J., Yoachim, P., \& Bernstein, R. A. 2004, ApJ, 608, 189

Dale, D. A., Cohen, S. A., Johnson, L. C., et al. 2009, ApJ, 703, 517

Dale, D. A., Gil de Paz, A., Gordon, K. D., et al. 2007, ApJ, 655, 863

Dame, T. M., Hartmann, D., \& Thaddeus, P. 2001, ApJ, 547, 792

de Blok, W. J. G., Walter, F., Brinks, E., et al. 2008, AJ, 136, 2648

Donovan Meyer, J., Koda, J., Momose, R., et al. 2012, ApJ, 744, 42

Downes, D., \& Solomon, P. M. 1998, ApJ, 507, 615

Draine, B. T., Dale, D. A., Bendo, G., et al. 2007, ApJ, 663, 866

Draine, B. T., \& Li, A. 2007, ApJ, 657, 810

Elfhag, T., Booth, R. S., Hoeglund, B., Johansson, L. E. B., \& Sandqvist, A. 1996, A\&AS, 115, 439

Elmegreen, B. G. 2011, in EAS Publications Series, Vol. 51, ed. C. Charbonnel \& T. Montmerle, 31

Feldmann, R., Gnedin, N. Y., \& Kravtsov, A. V. 2011, ApJ, 732, 115

Feldmann, R., Gnedin, N. Y., \& Kravtsov, A. V. 2012, ApJ, 747, 124

Foyle, K., Rix, H.-W., Walter, F., \& Leroy, A. K. 2010, ApJ, 725, 534

Fukui, Y., \& Kawamura, A. 2010, ARA\&A, 48, 547

Fukui, Y., Kawamura, A., Minamidani, T., et al. 2008, ApJS, 178, 56

Gao, Y., \& Solomon, P. M. 2004, ApJ, 606, 271

García-Burillo, S., Usero, A., Alonso-Herrero, A., et al. 2012, A\&A, 539, A8

Genzel, R., Tacconi, L. J., Combes, F., et al. 2012, ApJ, 746, 69

Genzel, R., Tacconi, L. J., Gracia-Carpio, J., et al. 2010, MNRAS, 407, 2091

Gil de Paz, A., Boissier, S., Madore, B. F., et al. 2007, ApJS, 173, 185

Glover, S. C. O., \& Mac Low, M. 2011, MNRAS, 412, 337

Heiderman, A., Evans, N. J., II, Allen, L. E., Huard, T., \& Heyer, M. 2010, ApJ, 723, 1019

Helfer, T. T., Thornley, M. D., Regan, M. W., et al. 2003, ApJS, 145, 259

Heyer, M., Krawczyk, C., Duval, J., \& Jackson, J. M. 2009, ApJ, 699, 1092

Heyer, M. H., Corbelli, E., Schneider, S. E., \& Young, J. S. 2004, ApJ, 602,723

Hughes, A., Meidt, S. E., Schinnerer, E., et al. 2013, arXiv:1304.1219

Hughes, A., Wong, T., Ott, J., et al. 2010, MNRAS, 406, 2065

Isobe, T., Feigelson, E. D., Akritas, M. G., \& Babu, G. J. 1990, ApJ, 364, 104

Israel, F. P. 1997, A\&A, 328, 471

Israel, F. P. 2009a, A\&A, 493, 525

Israel, F. P. 2009b, A\&A, 506, 689

Jogee, S., Scoville, N., \& Kenney, J. D. P. 2005, ApJ, 630, 837

Kawamura, A., Mizuno, Y., Minamidani, T., et al. 2009, ApJS, 184, 1

Kennicutt, R. C., Calzetti, D., Aniano, G., et al. 2011, PASP, 123, 1347

Kennicutt, R. C., \& Evans, N. J. 2012, ARA\&A, 50, 531

Kennicutt, R. C., Jr. 1989, ApJ, 344, 685

Kennicutt, R. C., Jr. 1998a, ARA\&A, 36, 189

Kennicutt, R. C., Jr. 1998b, ApJ, 498, 541

Kennicutt, R. C., Jr., Armus, L., Bendo, G., et al. 2003a, PASP, 115, 928

Kennicutt, R. C., Jr., Bresolin, F., \& Garnett, D. R. 2003b, ApJ, 591, 801

Kennicutt, R. C., Jr., Calzetti, D., Walter, F., et al. 2007, ApJ, 671, 333

Kewley, L. J., \& Ellison, S. L. 2008, ApJ, 681, 1183

Koda, J., Scoville, N., Sawada, T., et al. 2009, ApJL, 700, L132

Krumholz, M. R., Leroy, A. K., \& McKee, C. F. 2011, ApJ, 731, 25

Kuno, N., Sato, N., Nakanishi, H., et al. 2007, PASJ, 59, 117

Lada, C. J., Forbrich, J., Lombardi, M., \& Alves, J. F. 2012, ApJ, 745, 190

Lada, C. J., Lombardi, M., \& Alves, J. F. 2010, ApJ, 724, 687

Leroy, A., Bolatto, A. D., Simon, J. D., \& Blitz, L. 2005, ApJ, 625, 763

Leroy, A. K., Bigiel, F., de Blok, W. J. G., et al. 2012, AJ, 144, 3

Leroy, A. K., Bolatto, A., Bot, C., et al. 2009, ApJ, 702, 352

Leroy, A. K., Bolatto, A., Gordon, K., et al. 2011, ApJ, 737, 12

Leroy, A. K., Walter, F., Brinks, E., et al. 2008, AJ, 136, 2782

Liszt, H. S., Pety, J., \& Lucas, R. 2010, A\&A, 518, A45

Liu, G., Koda, J., Calzetti, D., Fukuhara, M., \& Momose, R. 2011, ApJ, 735, 63

Maloney, P., \& Black, J. H. 1988, ApJ, 325, 389

Marble, A. R., Engelbracht, C. W., van Zee, L., et al. 2010, ApJ, 715, 506
Meidt, S. E., Schinnerer, E., Garcia-Burillo, S., et al. 2013, arXiv:1304.7910

Meidt, S. E., Schinnerer, E., Knapen, J. H., et al. 2012, ApJ, 744, 17

Moustakas, J., Kennicutt, R. C., Jr., Tremonti, C. A., et al. 2010, ApJS, 190, 233

Muñoz-Mateos, J. C., Gil de Paz, A., Boissier, S., et al. 2009, ApJ, 701, 1965

Murgia, M., Crapsi, A., Moscadelli, L., \& Gregorini, L. 2002, A\&A, 385, 412

Murray, N. 2010, arXiv e-prints

Narayanan, D., Krumholz, M. R., Ostriker, E. C., \& Hernquist, L. 2012, MNRAS, 421, 3127

Oka, T., Hasegawa, T., Sato, F., et al. 2001, ApJ, 562, 348

Onodera, S., Kuno, N., Tosaki, T., et al. 2010, ApJL, 722, L127

Ostriker, E. C., McKee, C. F., \& Leroy, A. K. 2010, ApJ, 721, 975

Prescott, M. K. M., Kennicutt, R. C., Jr., Bendo, G. J., et al. 2007, ApJ, 668, 182

Press, W. H., Teukolsky, S. A., Vetterling, W. T., \& Flannery, B. P. (ed.) 1992, Numerical Recipes in C: The Art of Scientific Computing (Cambridge: Cambridge Univ. Press)

Prugniel, P., \& Heraudeau, P. 1998, A\&AS, 128, 299

Rahman, N., Bolatto, A. D., Wong, T., et al. 2011, ApJ, 730, 72

Rahman, N., Bolatto, A. D., Xue, R., et al. 2012, ApJ, 745, 183

Rebolledo, D., Wong, T., Leroy, A., Koda, J., \& Donovan Meyer, J. 2012, ApJ, 757, 155

Roman-Duval, J., Jackson, J. M., Heyer, M., Rathborne, J., \& Simon, R. 2010, ApJ, 723, 492

Rosolowsky, E., \& Blitz, L. 2005, ApJ, 623, 826

Rownd, B. K., \& Young, J. S. 1999, AJ, 118, 670

Saintonge, A., Kauffmann, G., Wang, J., et al. 2011, MNRAS, 415, 61

Saintonge, A., Tacconi, L. J., Fabello, S., et al. 2012, ApJ, 758, 73

Sakamoto, K., Okumura, S. K., Ishizuki, S., \& Scoville, N. Z. 1999, ApJS, 124,403

Sanders, D. B., \& Mirabel, I. F. 1996, ARA\&A, 34, 749

Sandstrom, K. M., Leroy, A. K., Walter, F., et al. 2012, arXiv:1212.1208

Schmidt, M. 1959, ApJ, 129, 243

Schmidt, M. 1963, ApJ, 137, 758

Schruba, A., Leroy, A. K., Walter, F., Sandstrom, K., \& Rosolowsky, E. 2010, ApJ, 722, 1699

Schruba, A., Leroy, A. K., Walter, F., et al. 2011, AJ, 142, 37

Schruba, A., Leroy, A. K., Walter, F., et al. 2012, AJ, 143, 138

Schuster, K.-F., Boucher, C., Brunswig, W., et al. 2004, A\&A, 423, 1171

Schuster, K. F., Kramer, C., Hitschfeld, M., Garćı-Burillo, S., \& Mookerjea, B. 2007, A\&A, 461, 143

Sheth, K., Regan, M., Hinz, J. L., et al. 2010, PASP, 122, 1397

Sheth, K., Vogel, S. N., Regan, M. W., Thornley, M. D., \& Teuben, P. J. 2005, ApJ, 632, 217

Solomon, P. M., Rivolo, A. R., Barrett, J., \& Yahil, A. 1987, ApJ, 319, 730

Strong, A. W., \& Mattox, J. R. 1996, A\&A, 308, L21

Tacconi, L. J., Genzel, R., Neri, R., et al. 2010, Natur, 463, 781

Tan, J. C. 2000, ApJ, 536, 173

Taylor, C. L., Kobulnicky, H. A., \& Skillman, E. D. 1998, AJ, 116, 2746

Verley, S., Corbelli, E., Giovanardi, C., \& Hunt, L. K. 2010, A\&A, 510, A64

Walter, F., Brinks, E., de Blok, W. J. G., et al. 2008, AJ, 136, 2563

Warren, B. E., Wilson, C. D., Israel, F. P., et al. 2010, ApJ, 714, 571

Watson, L. C., Martini, P., Lisenfeld, U., et al. 2012, ApJ, 751, 123

Wilson, C. D., Warren, B. E., Israel, F. P., et al. 2009, ApJ, 693, 1736

Wolfire, M. G., Hollenbach, D., \& McKee, C. F. 2010, ApJ, 716, 1191

Wong, T. 2009, ApJ, 705, 650

Wong, T., \& Blitz, L. 2002, ApJ, 569, 157

Wong, T., Hughes, A., Ott, J., et al. 2011, ApJS, 197, 16

Wu, J., Evans, N. J., II, Gao, Y., et al. 2005, ApJL, 635, L173

Young, J. S., Allen, L., Kenney, J. D. P., Lesser, A., \& Rownd, B. 1996, AJ, 112, 1903

Young, J. S., Kenney, J. D., Tacconi, L., et al. 1986, ApJL, 311, L17

Young, J. S., \& Knezek, P. M. 1989, ApJL, 347, L55

Young, J. S., \& Scoville, N. Z. 1991, ARA\&A, 29, 581

Young, J. S., Xie, S., Tacconi, L., et al. 1995, ApJS, 98, 219

Zibetti, S., Charlot, S., \& Rix, H. 2009, MNRAS, 400, 1181 